\title{
Neuromuscular adaptations induced by long-term motor skill training
}

Dissertation zur Erlangung des mathematisch-naturwissenschaftlichen Doktorgrades

\author{
"Doctor rerum naturalium" \\ der Georg-August-Universität Göttingen \\ im Promotionsprogramm \\ GAUSS Basisprogramm Biologie \\ der Georg-August University School of Science (GAUSS) \\ vorgelegt von \\ Robin Diedrichs \\ aus Salzgitter-Bad
}

Göttingen, 2018 



\section{Thesis Committee}

Prof. Ralf Heinrich, Department of Cellular Neurobiology, Schwann-Schleiden

Research Centre, Georg-August-University Göttingen

Prof. Dario Farina, Neurorehabilitation Engineering, Department of Bioengineering, Imperial College London

Prof. Deborah Falla, Rehabilitation Science and Physiotherapy, University of Birmingham

\section{Members of the Examination Board}

Referee: Prof. Ralf Heinrich, Department of Cellular Neurobiology, SchwannSchleiden Research Centre, Georg-August-University Göttingen

$2^{\text {nd }}$ Referee: Prof. Dario Farina, Neurorehabilitation Engineering, Department of Bioengineering, Imperial College London

\section{Further Members of the Examination Board}

Prof. Andreas Stumpner Department of Cellular Neurobiology, Schwann-Schleiden Research Centre, Georg-August-University Göttingen

Prof. Hansjörg Scherberger, Research Group Neurobiology, German Primate Center, Göttingen

Prof. Nils Brose, Department of Molecular Neurobiology, Max Planck Institute of Experimental Medicine, Göttingen

Ph.D. Hauke Werner, Research Group Neurochemistry, Department of Neurogenetics, Max Planck Institute of Experimental Medicine, Göttingen

Date of Oral Examination: October $11^{\text {th }}, 2018$ 
I hereby declare that this doctoral thesis entitled "Neuromuscular adaptations induced by long-term motor skill training" has been written independently with no other sources and aids than those quoted

Robin Diedrichs

August $28^{\text {th }}, 2018$

Göttingen, Germany 


\section{Table of Contents}

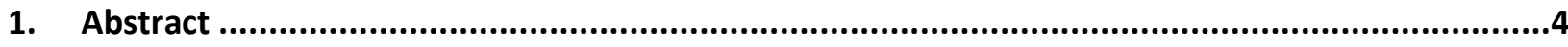

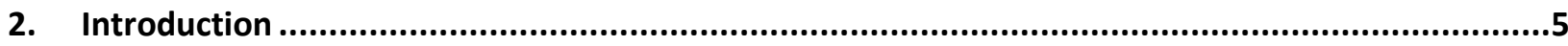

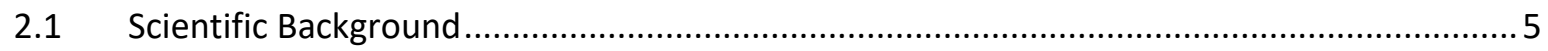

2.1.1 Significance and General Background................................................................... 5

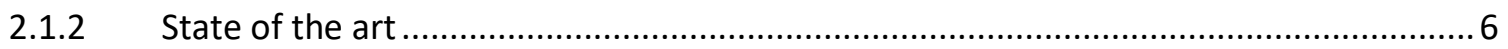

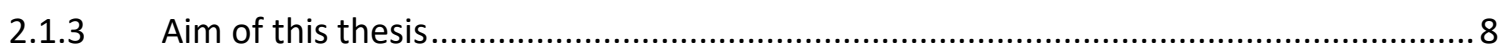

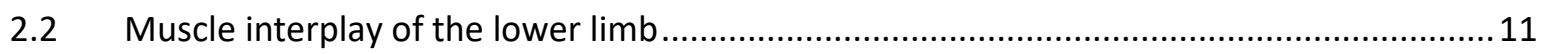

2.2.1 Muscle interplay - Agonist \& Antagonist................................................................. 11

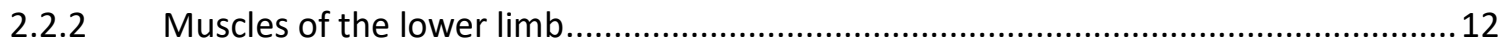

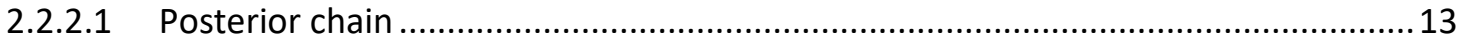

2.3 Central and peripheral nervous system ....................................................................... 14

2.3.1 The fundamental role of CNS and PNS in mammalian movement .............................14

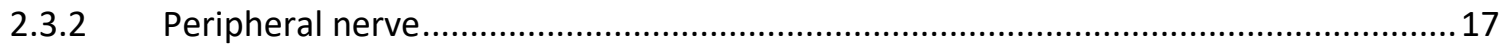

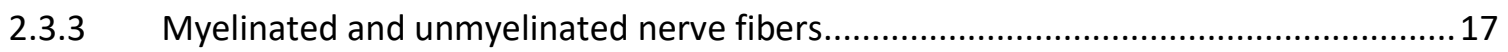

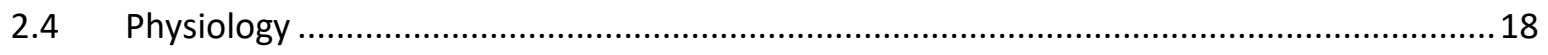

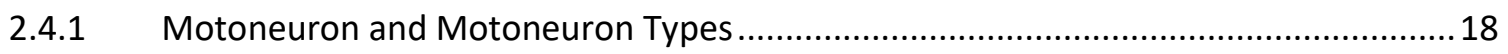

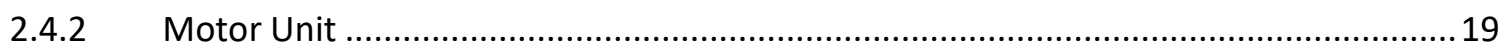

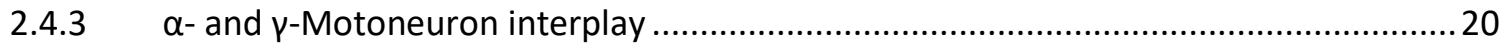

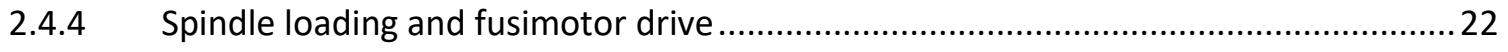

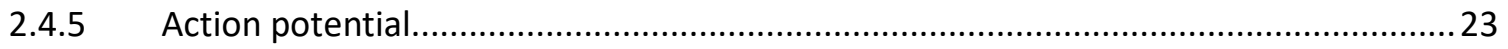

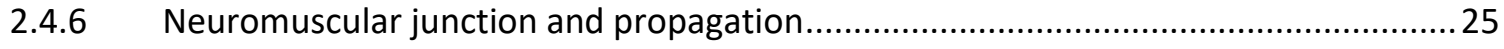

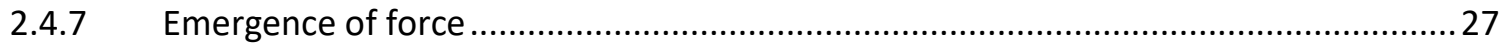

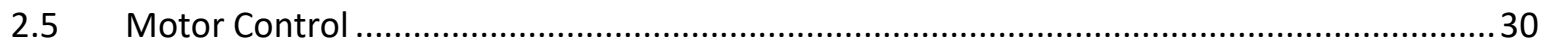

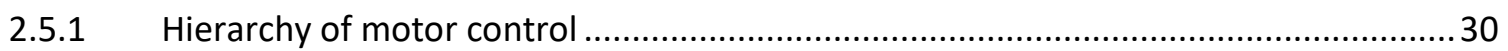

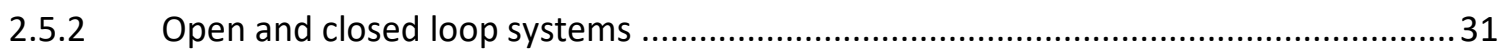

2.5.3 Feed-forward inhibition and feedback inhibition ........................................................ 32

2.5.4 Neurons' sensory and proprioceptive feedback system.............................................. 32

2.5.5 Muscle spindle and its role in motor control.............................................................. 35

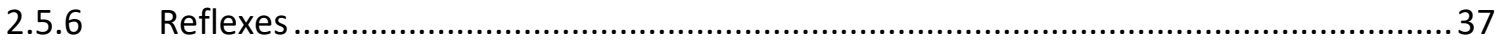

2.5.7 The spinal circuitry between lower limb extensor and flexor muscles .........................39

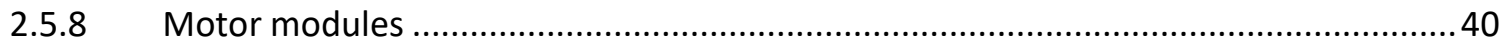




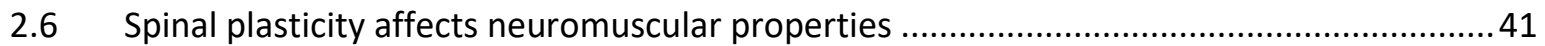

2.6.1 Investigation of motoneuron excitability and spinal plasticity ................................... 41

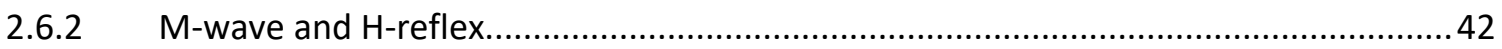

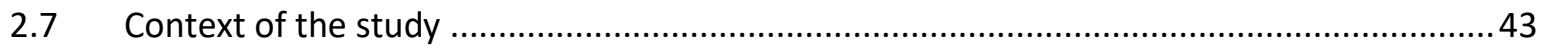

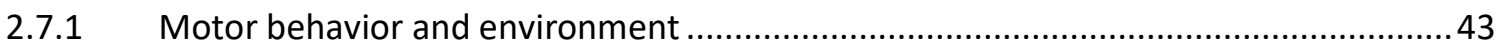

2.7.2 The effect of training on spinal plasticity .................................................................... 44

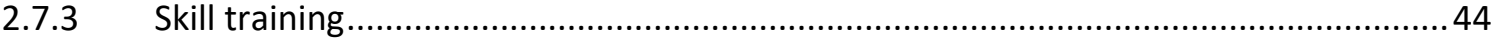

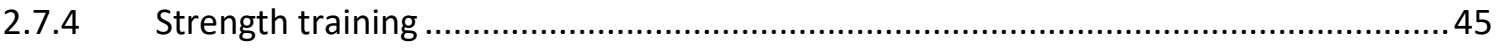

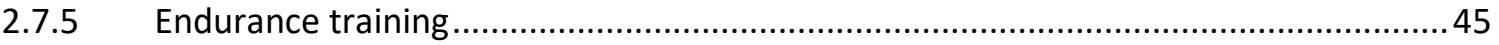

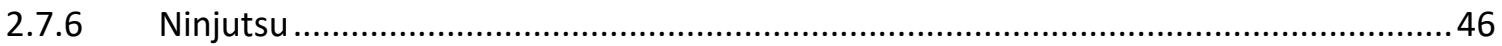

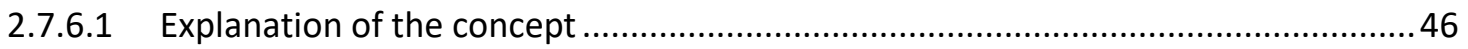

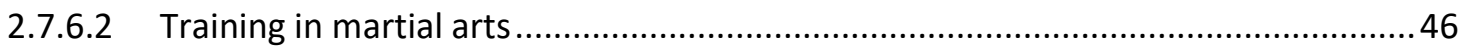

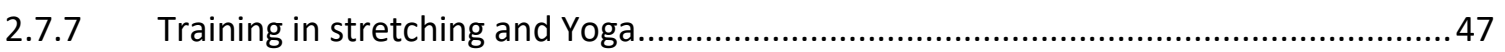

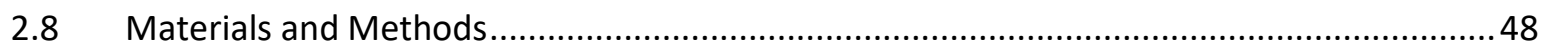

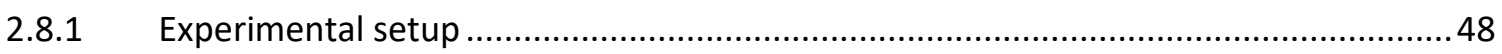

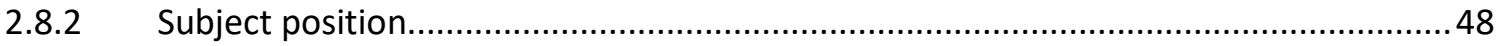

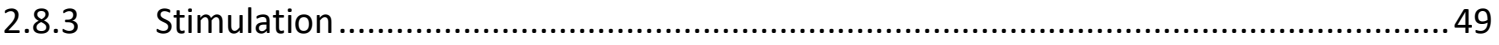

2.8.4 Electrode preparation and EMG recording .......................................................... 50

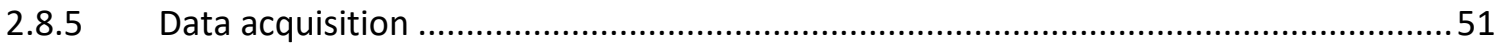

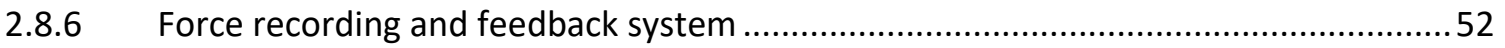

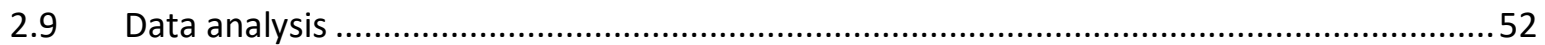

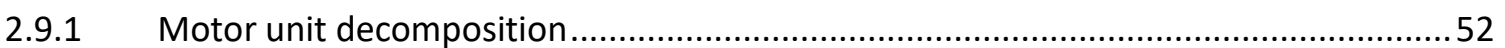

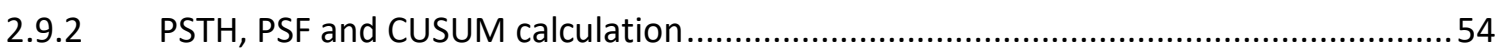

2.9.3 Estimation of recruitment threshold and reflex distribution ........................................ 56

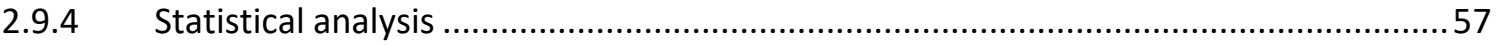

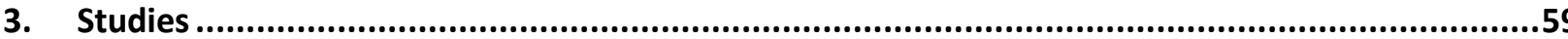

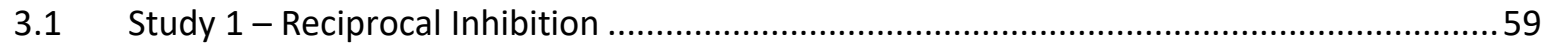

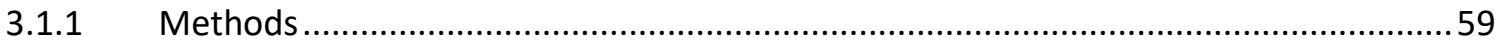

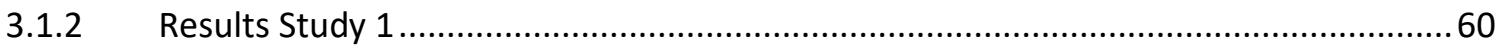

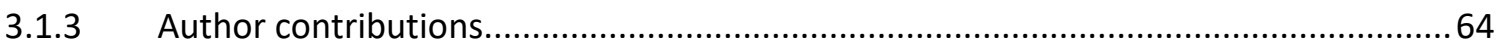

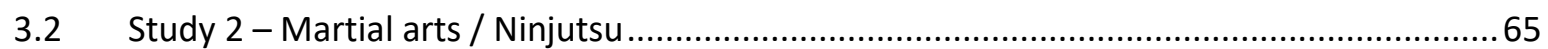

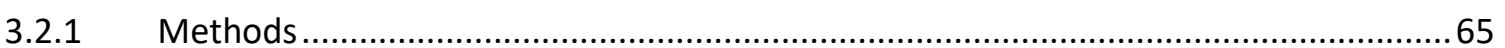

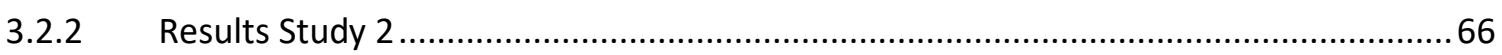

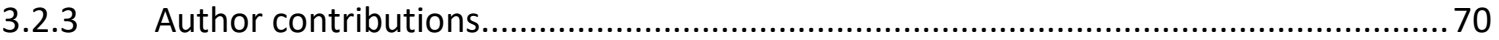




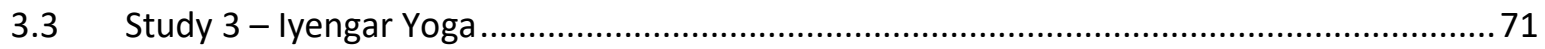

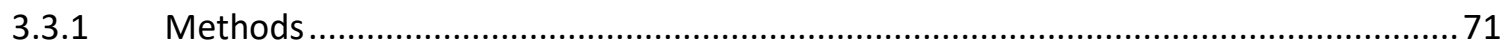

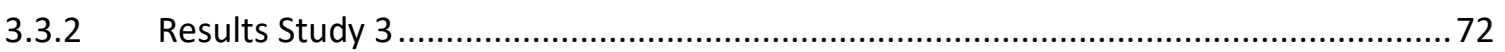

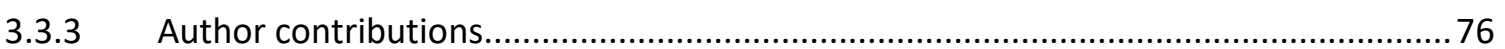

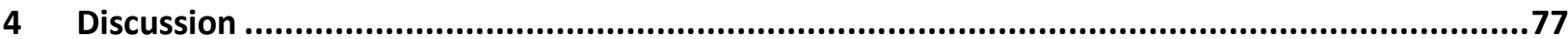

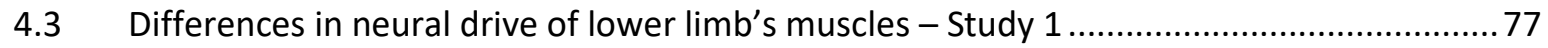

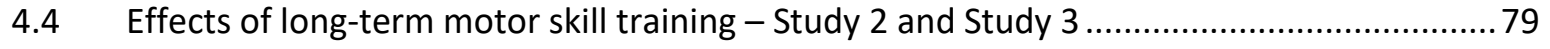

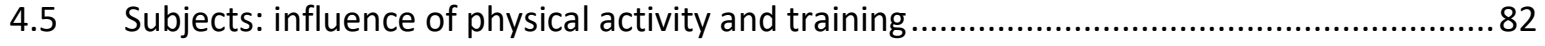

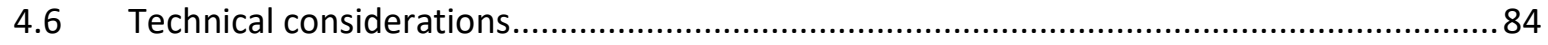

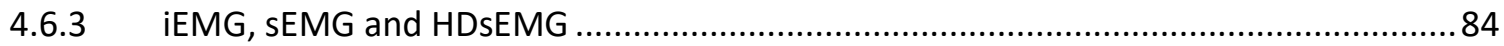

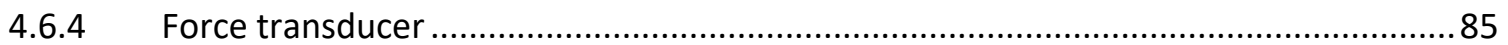

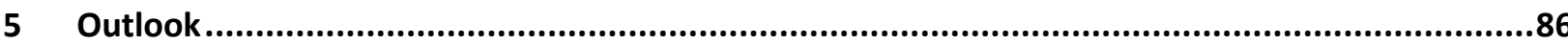

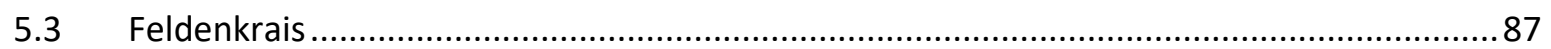

5.4 Further experiments - Consolidation and Reconsolidation ....................................... 88

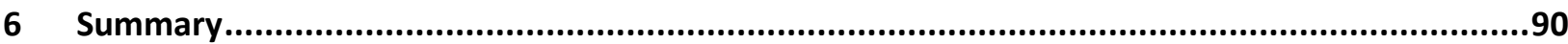

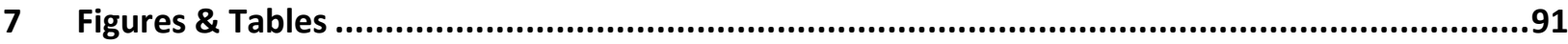

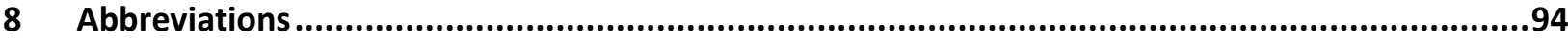

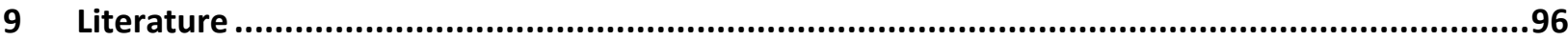

10 Curriculum Vitae.............................................................................................. 107

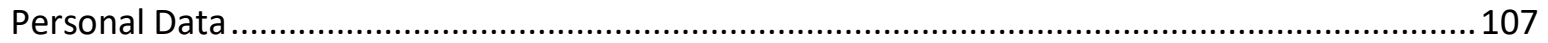

Education \& Professional/Work Experience................................... Error! Bookmark not defined.

Organization \& Representation ................................................. Error! Bookmark not defined.

Teaching Experiences............................................................ Error! Bookmark not defined.

Qualifications \& Experience ..................................................... Error! Bookmark not defined.

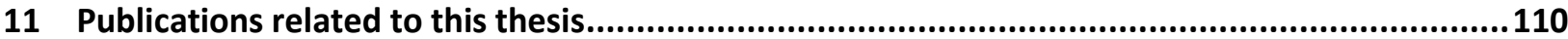

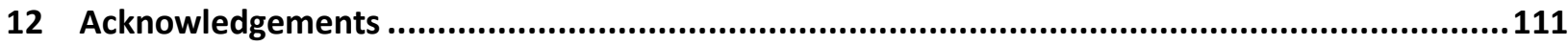


Abstract

\section{Abstract}

This thesis addresses the question of whether spinal cord's monosynaptic circuitry can be altered due to long-term motor skill acquisition. We researched functional organization and skill-related adaptation in muscle spindle feedback distribution. In study 1 we examined reciprocal inhibition of lower limb's extensor and flexor muscles and the underlying reflex arcs. After examining the interplay of lower limbs' extensor and flexor muscles, we aimed to investigate the effects of longterm motor skill training in a group of martial artists (study 2) and Yoga practitioners (study 3), compared to moderately active control groups. We approach these effects by investigation of neural input of $I_{A}$ afferent fiber onto spinal cords motoneuron pool.

Since several decades, resulting adaptations with regard to long-term motor skill acquisition are researched with intracellular recordings in model organisms and in humans. This brought up evidence to concept experiments with a non-invasive approach to analyze motor unit activity via $\mathrm{H}$ reflex measurements. The technique of Hoffmann-reflex (H-reflex) allows standardized excitation of $I_{A}$ afferent fibers while analyzing large populations of motor units. The technique of high-density electromyography-recording and H-reflex gives opportunity to evaluate high counts of firing motoneurons. This gives us the opportunity to analyze their firing behavior.

Within study 1 we found significant differences in lower limb's extensor ( $M$. soleus and $M$. gastrocnemius) and flexor (M. tibialis anterior) muscles' reciprocal inhibitory reflex amplitudes. Our findings support the hypothesis that lower limb's extensor and flexor muscle interplay is distributed asymmetrically; triceps surae muscles inhibit reflexes of $M$. tibialis anterior strongly. Furthermore we found significant differences in lower limb's extensor muscles' excitatory H-reflex amplitudes between groups of long-term trained martial artists and a moderately active control group (study 2 ). Our findings in a group of long-term trained yoga practitioners (study 3) were not significant, but indicate a trend with regard to neuromuscular and functional adaptations due to training experience. 


\section{Introduction}

\subsection{Scientific Background}

\subsubsection{Significance and General Background}

Body movement in everyday life requires fast adaptations in particular tasks to interact with changes of the environment. Motor behavior itself is modified due to musculoskeletal adaptations, at cortical and spinal level, and refines particular movements by changing the connectivity between neural assemblies (Casabona et al., 1990; Nielsen et al., 1993 b; Carp et al., 1995; Augé et al., 2000; Koceja et al., 2004; Adkins et al., 2006; Ogawa et al., 2009). These adaptations persist long-term, enabling the organism to adapt to its environment.

Extensive research on cortical plasticity in the context of motor learning has been performed in the last decades (Pascual-Leone et al., 1993; Carp et al., 1994, 1995; Remple et al., 2001; Wolpaw, 2007; Schubert et al., 2008; Vila-Cha et al., 2012). Animal studies show evidence that skill training alone induces changes of neural assemblies in the cortex and synaptogenesis (Adkins et al., 2006). Furthermore, it was shown that these changes in the motor cortex are evident only as a function of skill acquisition (Klintsova et al., 2004). Insights into neural plasticity of the human motor cortex due to skill training were gained with transcranial magnetic stimulation (Pascual-Leone et al., 1993; Tyč et al., 2005); reflex studies investigated changes in human reflex circuitry as a result of motor skill acquisition (Casabona et al., 1990; Nielsen et al., 1993 b; Augé et al., 2000; Koceja et al., 2004; Ogawa et al., 2009; Hirano et al., 2015; Chen et al., 2016). Acquisition of motor skill is thought to change feedback input gain; cortical plasticity is altered in terms of long-term motor skill training (Adkins et al., 2006).

The amount of contraction force is controlled by reflex loops (Kandel et al., 2012). By sensing the length of muscle fibers and tendons, information is provided about the body position in physical space and tactile information about the objects and the environment enclosing our body. Voluntary motor control is modulated by integration of sensory information of many different receptors to provide safe and desired movements (Kandel et al., 2012).

Postural muscles exhibit the highest amount of muscle spindles (Prochazka et al., 2012) and, with complexity of the task, the gain of afferent muscle spindle is affected, which makes the muscle spindle feedback to the main contributor of fine muscle interplay and dexterity (Pierrot-Deseilligny et al., 2012). The acquisition of skill related neuromuscular adaptations is thought to be mainly achieved by muscle spindle feedback (Thompson et al., 2009). The studies within this thesis aimed to 
Introduction

examine functional organization and skill based adaptation in muscle spindle input to individual motor units (MU).

Insights on how skill related neural plasticity alters neural structures on cortical level were achieved with several approaches. We know that the primary motor cortex is built up by neural assemblies that are heavily interconnected. This interconnectivity leads to precise movement of different and multiple joints (Adkins et al., 2006). Long-term motor skill training seems to change synaptic strength, synapse number by synaptogenesis and topography of stimulation evoked movement representations (Adkins et al., 2006); skill training is thought to affect the neural structures in a way that leads to reorganization of movement-representing neural assemblies (Adkins et al., 2006). Targeting spinal cord's neural plasticity, with regard to long-term motor skill training, we have chosen an investigation method which allows examination of reflex amplitudes of individual motor units; this approach gives us the possibility to investigate, in a simple reflex arc, neural drive during H-reflex (Yavuz et al., 2015, 2017).

Our approach provides the possibility to analyze the mutual distribution of reciprocal inhibitory input of lower limb's extensor and flexor muscles; the excitability of the motoneuron pool and subsequent reciprocal inhibition is thought to be determined by synaptic input distribution and intrinsic properties of $\alpha$-motoneurons (Yavuz et al., 2018). Direct investigation of the reciprocal inhibitory pathway is only possible by analyzing discharge timings of individual motor units and sequent statistics of a large number of motor units (Yavuz et al., 2018).

\subsubsection{State of the art}

Hoffmann reflex (H-reflex) has been utilized as a probe to estimate the excitability of the motoneuron pool (Pierrot-Deseilligny et al., 2000; Misiaszek, 2003; Knikou, 2008; Oliveira et al., 2012; Yavuz et al., 2014, 2015). H-reflex amplitudes indicate the excitability level of motoneurons and are therefore used to estimate muscles' neural drive by motoneurons; they demonstrate spinal plasticity in neural circuitries (Knikou, 2008). The H-reflex amplitude is defined as difference in height between first deflection of PSF-CUSUM (considered as reflex-response-onset) and subsequent peak (Yavuz et al., 2015). It represents the monosynaptic reflex activity in the spinal cord and is used to measure the response of the nervous system to various neurologic conditions, musculoskeletal injuries, application of therapeutic modalities, pain, exercise training and performance of motor tasks (Palmieri et al., 2004). An increase in H-reflex amplitude due to training experience, compared to a control group, represents a higher central gain of the monosynaptic stretch reflex, determined 
Introduction

by transmission across the synapses of the $I_{A}$ afferents and by excitability of the motoneuron pool (Nielsen et al., 1993 b).

From a technical point of view, we must consider that a reduction in H-reflex amplitude can be caused by high stimulation values. Afferent fibers are cutaneous electrically excited according to their size: from largest to smallest (Table 1). The threshold for type II fibers is around 2 - 5 times higher compared to type I fibers (Kandel et al., 2012). The resulting surface electromyography (sEMG) recording shows a relation of direct motor response (M-wave) to H-reflex. Low stimulation intensities excite mostly $I_{A}$ afferents and lead to orthodromic propagation along afferent fibers, which in turn causes excitation of $\alpha$-motoneurons of the homonymous muscle on spinal level (Pierrot-Deseilligny et al., 2012). Ortho- and antidromic propagation occur with high stimulation intensities, leading to direct excitation of $\alpha$-motoneurons (direct motor response) (Knikou, 2008). Since both $I_{A}$ afferents and $\alpha$-motoneurons are excited in this scenario, the physiologically elicited signal by the spinal cord collides with the elicited signal by electrical stimulation of $\alpha$-motoneurons which in turn causes extinction (Knikou, 2008). Amplitude modulation of clear H-reflexes without direct motor response ( $\mathrm{M}$-wave) is a sign of a change in neural drive which can derive from different synaptic input (Nielsen et al., 1993 b; Knikou, 2008; Yavuz et al., 2014); the onset of the H-reflex amplitude in dependency of time after the stimulation is considered as latency and correlates with the leg lengths (Falco et al., 1994). On the other hand, M-wave recordings by supramaximal electrical stimulation intensities can be used to analyze differences in the temporal onset of M-wave amplitude between subjects (Cavanagh et al., 1979; Yavuz et al., 2010).

Since the H-reflex is a useful tool to represent the excitability of the monosynaptic reflex circuitry after a proceeded excitation of peripheral afferents, it gives us insight into spinal neuroplasticity by information of muscle spindle afferents. Nielsen et al. (Nielsen et al., $1993 \mathrm{~b}$ ) showed that H-reflex of M. soleus (SOL), due to long-term adaptation, differs significantly between a group of dancers of the Royal Danish Ballet and well-trained athletes. The amount of $H_{\max } / \mathrm{M}_{\max }$ (maximum H-reflex amplitude divided by maximum M-wave amplitude) is significantly larger in trained athletes but smaller in ballet dancers (Nielsen et al., 1993 b). Previous studies show a decrease in SOL H-reflex amplitude and bisynaptic reciprocal inhibition (Nielsen et al., 1993 b; Perez et al., 2007) as well as an increase in H-reflex amplitude (Schneider et al., 2002; Ogawa et al., 2009) caused by adaptations with regard to motor-skill training. Perez et al. (Perez et al., 2007) showed that a co-contraction training exercise of 30 min leads to a decrease in H-reflex amplitude, which is thought to derive from changes in corticospinal and reflex excitability. In 2009, Ogawa et al. (Ogawa et al., 2009) showed, with respect to long-term swimming training, an increased gain of SOL stretch reflex as well as 
Introduction

increased H-reflex compared to a non-trained control group. Findings by Schneider \& Capaday (Schneider et al., 2002) showed an increase in H-reflex amplitude in different phases of walking backwards due to training. Their results indicate that daily training in walking backwards causes more security in walking backwards, leading to a decrease in SOL H-reflex in mid-swing phase of walking backwards (Schneider et al., 2002).

We focused on spinal plasticity in skill acquisition since the spinal cords' "comparative simplicity, accessibility and its well-defined connections with the brain facilitate explorations of activitydependent plasticity, and of the ways in which multiple sites of plasticity interact to produce a new skill" (Wolpaw, 2007). Prior investigations of neuromuscular changes in long-term motor skill trained martial artists revealed only differences in kinetics and global surface EMG measurements (Sørensen et al., 1996; Zehr et al., 1997; Sforza et al., 2002; Arus, 2013; Zaggelidis et al., 2013). With the presented approach it is also possible, due to differences in electro-mechanical delay (EMD), to define a time-difference in muscle activation; EMD is similar between gender, but longer in elderly compared to younger human (Yavuz et al., 2010). Differences in EMD are thought to be caused by the required time to stretch muscles' serial elastic components (Cavanagh et al., 1979).

\subsubsection{Aim of this thesis}

Since it is accepted that skill training leads to refinement of neural assemblies (Adkins et al., 2006), we aimed in study 1 and study 2 to investigate spinal plasticity in long-term experienced athletic martial artists assessing the distribution of afferent inputs to motoneurons; long-term skill training results in adaptations of the neuromuscular system (Adkins et al., 2006). Various approaches were used to show differences between skilled and control group, but all investigations are based on global surface EMG (sEMG) recordings (Capaday et al., 1990; Nielsen et al., 1993 b; Augé et al., 2000; Ogawa et al., 2009). While using the technique of high-density electromyography-recording (HDsEMG), the behavior of a large amount of motor units can be estimated (Yavuz et al., 2014, 2015, 2017); the main advantage of HDsEMG-recordings and subsequent statistical analysis is the measurement of a large count of activated motoneurons (Yavuz et al., 2015). The technique of HDsEMG-recordings provides us unique possibility to demonstrate the behavior of a large amount of motor units as well as changes in the neuromuscular system between a group of long-term skilled participants and a control group.

We studied HDsEMG recordings from lower limb postural muscles, i.e. $M$. soleus and $M$. gastrocnemius (GM) (Farina et al., 2010; Holobar et al., 2014; Yavuz et al., 2014, 2015). Wire electrodes (i.e. iEMG) are highly selective; due to the small actively measuring tip of the electrode, 
Introduction

only a few muscle fibers are 'hooked' (Yavuz et al., 2015) and, in dependency of the insertion depth (Henriksson-Larsén et al., 1983; Schmidt et al., 2007; Milnik, 2012), different muscle fibers are recorded. In contrast, sEMG-recordings provide only information about the summation of all electrical signals, which propagate over the muscle belly. The variation of identified motor units through different recording sessions with HDsEMG is very small compared to iEMG-recording (Yavuz et al., 2014, 2015); the count of analyzed MUs in HDsEMG compared to iEMG is very high. With this approach, we are able to estimate neural drive of the motoneuron pool, and therefore it provides us the possibility to demonstrate the behavior of a large count of motor units, as well as differences in the neuromuscular system (Yavuz et al., 2015) between (i) lower limb's extensor and flexor muscle and (ii) a group of long-term skilled participants and a control group.

Recorded electrical information of HDsEMG electrodes represents mainly the activity of muscle fibers located in the near or on the muscle belly, whereas iEMG provides information about muscle fibers depending on the insertion depth; it must be taken into account that the surface of the muscle is rich in oxygen and contains more oxidative fibers (Henriksson-Larsén et al., 1983), whereas the inside of the muscle contains more glycolytic fibers (Schmidt et al., 2007; Milnik, 2012). Another advantage of HDsEMG recordings compared to intramuscular recordings is that participants are not affected by any discomfort or painful events due to a needle insertion.

Neuromuscular adaptations influenced by motor skill training occur in afferent as well as in efferent fibers. Muscle spindle responsiveness may be adjusted by motor learning (Pierrot-Deseilligny et al., 2012); $y$-motoneurons seem to adapt to a learnt co-contraction with increased firing, and to changes in muscle length (Nielsen et al., 1994). All movements are learnt, and afferent input feeds not only spinal cord but the sensorimotor cortex; it should be taken into account that $\gamma$-motoneurons' input to supraspinal centers maintain coherence of sensory input (Pierrot-Deseilligny et al., 2012). $\gamma$-Drive is thought to contribute with great significance to muscle spindle endings during slow contractions, like walking, that involve co-contraction (Pierrot-Deseilligny et al., 2012).

Afferent input by $I_{A}$-afferents to $\alpha$-motoneurons is altered by motor skill training, as several studies revealed (Casabona et al., 1990; Nielsen et al., 1993 b; Augé et al., 2000; Adkins et al., 2006; Perez et al., 2007; Ogawa et al., 2009; Pierrot-Deseilligny et al., 2012). Since $I_{A}$ afferent fibers project directly onto $\alpha$-motoneurons of the homonymous muscle, as well as onto interneurons of antagonistic muscles, muscle interplay is highly modified by skill acquisition; even sensorimotor cortex plays an important role for motor program planning (Kandel et al., 2012), which is modifiable by $I_{A}$ afferent input (Pierrot-Deseilligny et al., 2012). 
Introduction

$I_{B}$ input onto interneurons was thought to play a role in autogenic protective reflex, but further investigation led to the assumption that $I_{B}$ inhibition contributes to various pathways (PierrotDeseilligny et al., 2012); they connect with $\alpha$-motoneurons, $\gamma$-motoneurons, other $I_{B}$ interneurons and $I_{A}$ afferents. Input of interneurons is received by $I_{B}$ and $I_{A}$ afferents interposed by interneurons from group II, cutaneous, joint and interosseous afferents and are thought to contribute to feedback control (Pierrot-Deseilligny et al., 2012). While performing motor tasks, $I_{B}$ inhibition is altered, likely due to presynaptic inhibition of $\mathrm{I}_{\mathrm{B}}$ afferents, and is suppressed during homonymous contractions (Pierrot-Deseilligny et al., 2012).

Type II fibers are difficult to investigate, since they travel along the same fiber bundle as type I fibers; if they are activated, for instance by muscle stretch, primary endings are excited as well, due to their lower thresholds (Pierrot-Deseilligny et al., 2012). Secondary endings project weakly onto motoneurons of the homonymous muscle but mainly onto interneurons (Pierrot-Deseilligny et al., 2012). Spatial and temporal summation in excitatory postsynaptic potentials (ESPS) of interneurons is very little and transmission very effective; type II efferents contribute to $\alpha$ - and $\gamma$-motoneurons and descending tracts (Pierrot-Deseilligny et al., 2012). They seem to contribute to 'automatic' human walking by homonymous and heteronymous group II discharges (Pierrot-Deseilligny et al., 2012).

In this thesis we first aimed to investigate differences in reciprocal inhibitory reflex amplitudes between the lower limb muscle TA and triceps surae (TS) muscle group (Yavuz et al., 2018). The motor neuron pool's excitability is determined by intrinsic properties of $\alpha$-motoneurons and distribution of synaptic inputs (Miles et al., 1986; Kandel et al., 2012; Yavuz et al., 2018). I $I_{A}$ afferent fibers of the homonymous muscle are activated monosynaptic if muscle fibers are stretched (PierrotDeseilligny et al., 2000; Knikou, 2008); we know that $\mathrm{I}_{\mathrm{A}}$ afferents' input onto motoneurons depends on the amount of proprioceptive feedback (Knikou, 2008; Kandel et al., 2012), that it is depending on training-experiences (Nielsen et al., 1993 b; Schneider et al., 2002; Adkins et al., 2006; Mazzocchio et al., 2006; Vila-Cha et al., 2012; Sawers et al., 2015), and that it can be altered with operant conditioning (Wolpaw, 2006; Thompson et al., 2009, 2014). Co-contraction of muscles provides possibility to brace joints (Nielsen et al., 1992, 1993 a, 1994; Perez et al., 2007); fine muscle interplay enables the neuromuscular system to perform fine and coordinated movements (Schubert et al., 2008; Chvatal et al., 2013; Sawers et al., 2015). Within our studies, the participants performed sustained plantar flexion at different percentiles of their maximum voluntary contraction force. In this thesis we aimed first to study the interplay, i.e. reciprocal inhibition, between the calf muscles SOL \& GM (extensor muscles) and TA (flexor muscle), since asymmetry was found in investigations of 
Introduction

single MUs in human (Crone et al., 1987; Nielsen et al., 1992). In our second and third study we compared two groups with different motor experiences; we expect differences in excitatory H-reflex amplitudes between both groups, since previous studies strongly support our hypothesis for differences in SOL H-reflex amplitudes due to training experience (Nielsen et al., 1993 b; Mazzocchio et al., 2006; Perez et al., 2007; Vila-Cha et al., 2012). H-reflex is elicited by repetitive low intensity stimulation of tibial nerve (TN) in the popliteal fossa (Knikou, 2008; Yavuz et al., 2015). With this method we are able to estimate neural drive for excitatory and inhibitory neural circuits (De Luca et al., 2006; Farina et al., 2010; Yavuz et al., 2015, 2018); the large amount of identified MUs from the HDsEMG represents the motoneuron pool more accurate compared to single MU recordings (Yavuz et al., 2015).

In this light, we hypothesize that the excitatory and inhibitory postsynaptic potentials (EPSP and IPSPs) elicited from $I_{A}$ afferent fibers are distributed to motoneurons differently

- between lower limb's extensor and flexor muscles TA and TS muscles (study 1),

- between experienced athletes and a moderately active control group (study 2 and study 3 ),

This differential distribution is thought to be the result of a functional and neural re-organization of sensory input due to long-term motor skill training (Adkins et al., 2006); In this sense, differences between skilled and unskilled participants result in enhanced proprioceptive feedback and balance control (Schneider et al., 2002). We hypothesize that underlying neural circuitries adapted in a longterm motor-trained group due to their training experience.

\subsection{Muscle interplay of the lower limb}

\subsubsection{Muscle interplay - Agonist \& Antagonist}

Since a skeletal muscle is attached to the skeletal system with tendons which mostly traverse over joints, there are different types of contractions a muscle can perform. The different types of contraction are described as isotonic, isometric and auxotonic (Schmidt et al., 2007; Widmaier et al., 2013); eccentric contraction appears when the contracted muscle is lengthened; isotonic concentric contraction describes the shortening of muscle length while the muscle is already contracted (Arus, 2013). Subjects in all presented studies performed sustained isometric contractions for the estimation of reflex responses and associated examined parameters. 
Introduction

The agonist is functionally, respectively anatomically, defined as the muscle which contracts through its own excitation, where on the other hand the antagonist is the muscle which undertakes control for the opposite movement of the joint (Schiebler et al., 2007; Schmidt et al., 2007). Physiologically, agonist and antagonist are defined as muscles' interplay accomplished by neural circuits of afferent and efferent fibers via excitation and inhibition of muscles (Kandel et al., 2012). Since some tasks require rapid switch between agonist and antagonist activation or necessitate co-activation (Nielsen et al., 1993 b), the repertoire of recruiting strategies is broad (Kandel et al., 2012). Different pathways exist which base on activation and on reciprocal inhibition of the antagonistic muscle (Crone et al., 1987; Capaday et al., 1990; Yavuz et al., 2014, 2017). The terms for agonistic and antagonistic muscles exist due to their functionality in motion and show no intrinsic property itself.

\subsubsection{Muscles of the lower limb}

The muscles of the lower limb can be assigned into three categories (Schiebler et al., 2007):

- Ventral branch (extensors): These muscles include the M. tibialis anterior, extensor hallucis longus, extensor digitorum longus and fibularis (peroneus) tertius

- Lateral branch: These muscles are the M. fibularis (peroneus) longus, fibularis (peroneus) brevis

- Dorsal branch (flexors): the superficial layer consists of M. gastrocnemius, soleus and plantaris whereas the deep layer consists of M. popliteus, flexor digitorum longus, tibialis posterior and flexor hallucis longus

M. gastrocnemius (GM) is part of the dorsal branch of the leg. It is a skeletal muscle and consists of two heads with two origins that unite in a single tendon (c.f. Figure 1 and Figure 2). The medial head originates from the medial epicondyle of the femur. It is stronger and longer, compared to lateral head. It springs from the lateral epicondyle of the femur. The deeper layer and lower part of the muscle is composed of aponeurotic fascia for each section of the muscle and inserts to the calcaneus. The mutual tendon of GM and SOL is the Achilles' tendon. The calf muscles work synergistic and, since both muscles share the same tendon, different perspectives evolved (Schiebler et al., 2007), whether it is an isolated muscle or part of the triceps surae (TS) muscle group. The function of GM is plantar flexion of the ankle and assistance of knee flexion. Muscle fibers are longer compared to SOL which provides speed and short-lasting contractions. It is innervated by the tibial nerve (TN) (Schiebler et al., 2007). 


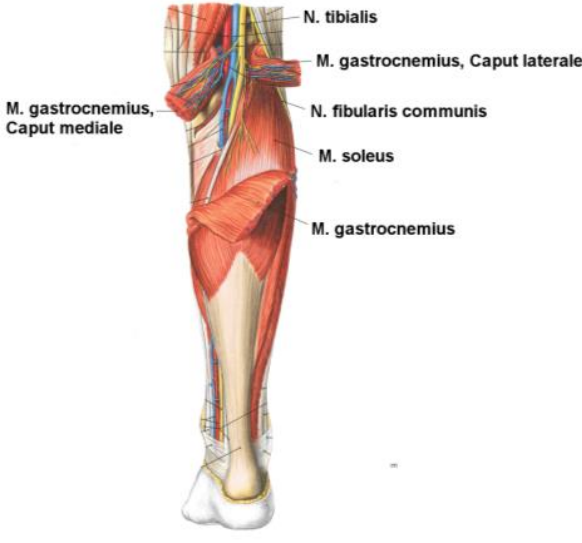

Figure 1: Depicts dorsal view of Regio cruris posterrior and Fossa poplitea right side. Fascia cruris removed and $M$. gastrocnemius dissected (by Sobotta et al., 2017, modified.)
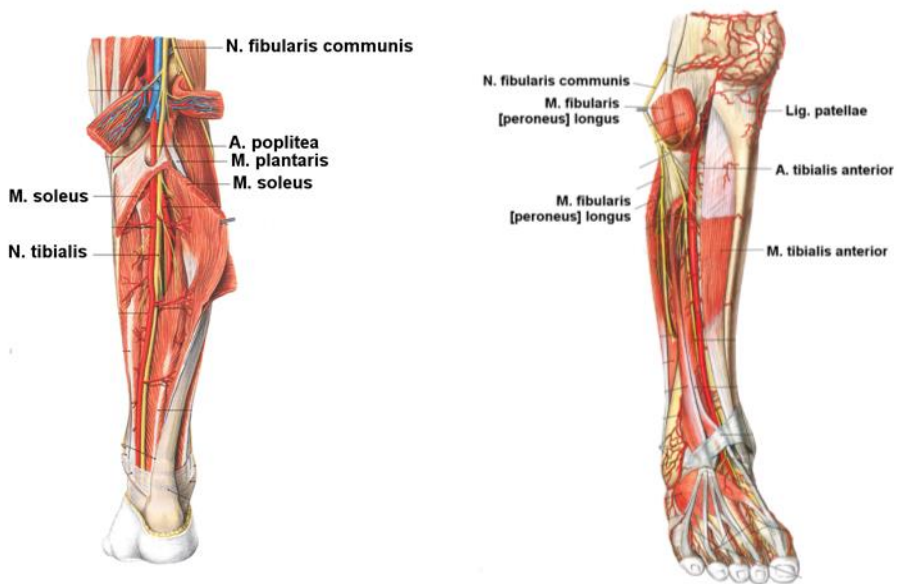

Figure 2: Depicts dorsal view of Figure 3: Depicts ventral view of Regio cruris posterrior and Fossa Regio cruris anterior, right side, poplitea right side. M. containing M. tibialis anterior (by gastrocnemius and M. soleus Sobotta et al., 2017, modified). dissected (by Sobotta et al., 2017, modified).

M. soleus (SOL) is, as well as GM (and M. plantaris), part of the dorsal branch. It is a skeletal muscle with its origin at the dorsal face of the fibula and on the tibia's soleal line. It is inserted on Calcaneus' dorsal surface together with GM's tendon (c.f. Figure 1 and Figure 2). Its function is plantar flexion of the ankle and maintenance of vertical erect position of the body. It is, as well as GM, innervated by the tibial nerve. Muscle fibers are shorter compared to GM. This provides enduring contractions and force (Schiebler et al., 2007).

M. tibialis anterior (TA) is part of the ventral branch and is a skeletal muscle. It is situated directly lateral next to the shin (Figure 3). It originates from the lateral condyle and the lateral shaft of the tibia or the upper two-thirds, the interosseus membrane and from the lateral intermuscular septum's deep fascia of the thigh. Tibialis muscle is the most powerful muscle that achieves ankle's dorsiflexion and achieves foot's inversion. It is innervated by deep peroneal nerve (common peroneal nerve, CPN). TA is partial agonist of $M$. tibialis posterior and antagonist of the $M$. peroneus longus (Schiebler et al., 2007).

\subsubsection{Posterior chain}

As posterior chain, a functional assembly of muscles is meant (Myers, 2013). This assembly consists mainly of the calf muscles, hamstrings, $m$. glutei, M. latissimus dorsi and the erector spinae muscle group; these muscles are big in appearance and can produce high amounts of constant force; the function of this muscle group is quite numerous (Schiebler et al., 2007; Arus, 2013). They contribute for example in running, jumping and changes of movement direction. They are anatomically taking 
Introduction

part in stabilization of knee, hip and spine (Schiebler et al., 2007). Muscles of the posterior chain are most often involved in lower back pain (Williams et al., 2005, 2009; Tilbrook et al., 2011; Dupeyron et al., 2013).

Besides our approach of gaining insights into neural adaptations induced by long-term motor skill training on physiological level, we also accessed kinetics to examine underlying changes for different performance in tests between skilled subjects and control group (c.f. chapter 4.4).

\subsection{Central and peripheral nervous system}

The central nervous system (CNS) is the part of the human nervous system, which consists of the brain itself and the spinal cord. The peripheral nervous system (PNS) consists of all nerve fibers which are not part of the CNS. Both are heavily connected with each other, but the PNS is subordinated to the CNS (Kandel et al., 2012). By definition nerve fibers in the CNS are myelinated by oligodendrocytes whereas in the PNS Schwann cells undertake the task of myelination (Schmidt et al., 2007). The task of the spinal cord can be generally described as the integration of sensory stimuli via afferents, coordination of involuntary motor functions such as reflexes and some regulation of vegetative processes. Voluntary movement, complex regulation of inner organs and integration of different sensory input involve higher brain functions. The interplay between brain and spinal cord is of great importance for complex movements (Kandel et al., 2012).

\subsubsection{The fundamental role of CNS and PNS in mammalian movement}

Kandel et al. (Kandel et al., 2012) describe the role of CNS in PNS in mammalian movement as follows:

The brain can be divided into different sections: brainstem, cerebellum, diencephalon and cerebrum. The brainstem's medulla represents an extension of the spinal cord. The medulla contains, beneath cranial nerve nuclei, the apneustic center and other core areas of neural control. The medulla composes together with pons and midbrain the brainstem. The cerebellum connects with all three parts of the brainstem. The diencephalon is defined anatomically or functionally whereby various definitions exist that include or exclude the hippocampus. Pons and midbrain have great importance in linking areas of the brain, in breathing, sleeping and taste (pons) and in visual and auditory (midbrain) signal processing. The cerebellum is located behind the pons. It is mainly involved in 
Introduction

movement coordination and control of posture. This structure coordinates the adaption of movement as well as specialization and perfection. The diencephalon contains the structures of thalamus and hypothalamus. Incoming signals via the PNS are sorted and integrated in this brain area. The hypothalamus is the area of primitive feelings like hunger and thirst. The largest visual part of the brain is the cerebrum with the cerebral hemispheres. Its function can be depicted as responsible for motor planning, perception, memory and emotion. The cortex of the cerebrum has great importance for planning and carrying out movement tasks.

Motor cortex describes a definable region of the cerebral cortex. The motor cortex itself is involved in execution of voluntary movements, motor control and motor planning. It is functionally subdivided into three areas: (i) The primary motor cortex responsible for movement execution creates the main drive together with other motor areas. Its neurons project directly onto $\alpha$ motoneurons in the spinal cord; (ii) The premotor cortex plays a main role in motor control, especially movement preparation, regarding dimensional and sensory planning and integration; (iii) The supplementary motor area (SMA) is less involved in direct planning of movements but in the planning and coordination of commands of the premotor cortex (Kandel et al., 2012) (c.f. Figure 4). Moreover, bi-manual planning and coordination is accomplished by the SMA. Further areas of the motor cortex are the posterior parietal cortex and the primary somatosensory cortex. Basal ganglia contribute to control of voluntary movement. The complex function of these core areas remains still unclear but is thought to contribute by selection and processing, respectively by inhibition or suppression, to motor tasks; another important role is its gating function in a control loop between thalamus, frontal lobe and cerebral cortex (Kandel et al., 2012). Midbrain is closely associated with the motor system. It is a part of the brainstem and located between pons and diencephalon. It is responsible for passing on of proprioceptive information of sensory fibers to the cerebrum (telencephalon). Also, sensory information is redirected onto motoneurons by the midbrain (Kandel et al., 2012).

The cerebellum supports movement by integration of impulses of the sensory system by the spinal cord. Sensory information of the spinal cord is processed and integrated in the cerebellum. By this, timing, precision and coordination of motor actions are improved (Kandel et al., 2012).

The spinal cord (Medulla spinalis) is the part of the CNS in vertebrates which spreads out from the brainstems' medulla oblongata to the lumbar region of the vertebral column. It is localized in the spinal canal and, as the brain itself, surrounded by the meninges. A major share of the peripheral system connects with the spinal cord via dorsal and ventral roots of the spinal canal (Kandel et al., 2012). It consists of grey and white matter (c.f. Figure 4). 


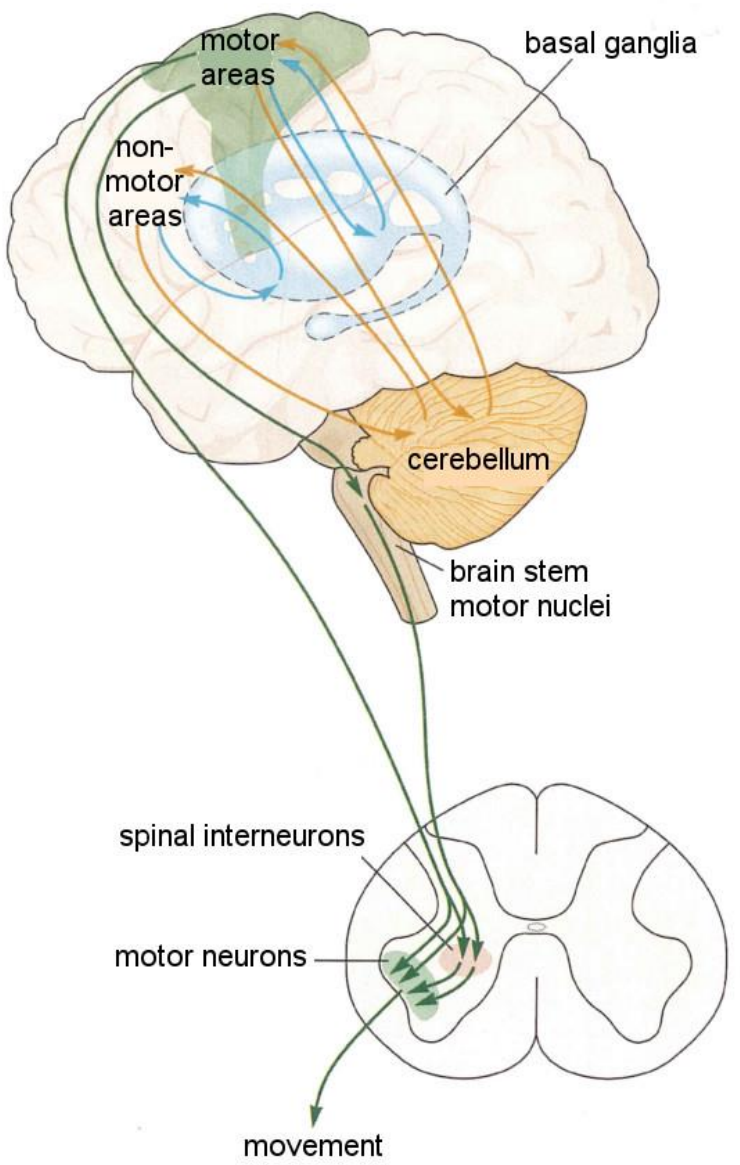

Figure 4: Explanation of the levels of motor control (forebrain, brain stem and spinal cord). Green arrows depict direct activation by motor areas or indirect by brain stem's descending systems issuing into spinal cord. Afferent input is received at all levels. Orange arrows show influence of cerebellum and basal ganglia, green arrows show direct motor commands by motor areas onto spinal interneurons and motoneurons., blue arrows indicate integration of information in basal ganglia (by Kandel et al., 2012, modified).

The CNS can be divided morphologically into the grey matter and white matter. While white matter contains the fibers, only a few myelinated axons are located in it; grey matter is one of the major components of the CNS, containing nerve cell bodies, dendrites, non-myelinated axons, glia cells and capillaries. In the spinal cord, grey matter is surrounded by white matter and an anterior and posterior horn of lateral ventricle forms a butterfly-like structure (Kandel et al., 2012), c.f. Figure 4.

On the contrary, white matter is composed mainly of myelinated axons that are bundled into tracts. The myelination of these surrounding glia cells and myelin sheaths appear optical white. Within spinal cord it surrounds grey matter and can be distinguished as an anterior, lateral and posterior branch (funiculus). In the cerebral cortex localization of matter is vice versa, compared to the spinal cord; here, white matter is surrounded by mainly grey matter (Kandel et al., 2012). 
Introduction

\subsubsection{Peripheral nerve}

A nerve is a bundle of axons outside the central nervous system, which contains efferent axons projecting to effectors and/or afferent axons from sensory cells. Peripheral nerves contain nerve fascicles and connective tissue. Each fiber is surrounded by endoneurium, whereas epineurum surrounds nerves and adipocytes. Some up to hundreds of nerve fibers are grouped by the perineum to bundles. These bundles are grouped by fascicles and form up the epineurum surrounded mixed nerve (Kandel et al., 2012).

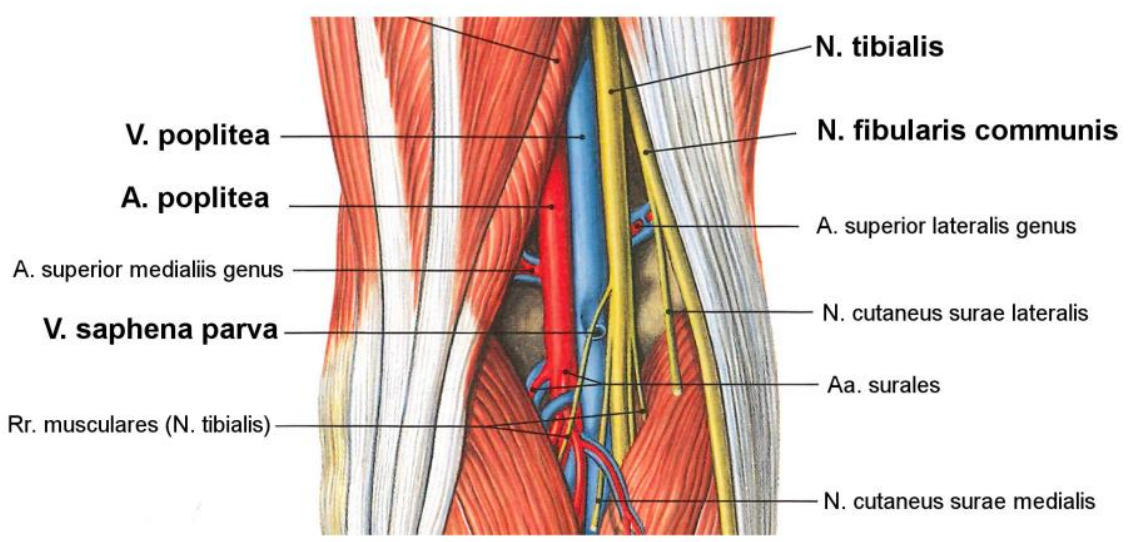

Figure 5: Popliteal fossa depicting stimulation area of the peripheral nerve (by Schmidt et al., 2007, modified).

In general, the afferent fibers are responsible for sensing in- and extrinsic properties, whereas efferent fibers lead to activation of target muscles. The neural pathways involved in postural control are quite complex and linked with each other. We know that these pathways can be altered due to long-term motor skill training (Rochcongar et al., 1979; Augé et al., 2000; Schneider et al., 2002; Adkins et al., 2006; Ogawa et al., 2009; Vila-Cha et al., 2012). It is of great necessity to deeply examine occurrence of neural adaptations on the level of single motor units, to determine which neural structures are altered with regard to short-, mid-, as well as long-term motor skill training.

\subsubsection{Myelinated and unmyelinated nerve fibers}

Table 1: Afferent fiber groups in peripheral nerves (by Kandel et al., 2012, modified). Classification of sensory afferents in muscle by their diameter, whereas sensory nerves in cutaneous nerves are classified according their conduction velocity:

\begin{tabular}{|c|c|c|c|c|c|}
\hline & & $\begin{array}{l}\text { Muscle } \\
\text { nerve }\end{array}$ & Cutaneous nerve & $\begin{array}{l}\text { Fiber diameter } \\
{[\mu \mathrm{m}]}\end{array}$ & $\begin{array}{l}\text { Conduction } \\
\text { Velocity }[\mathrm{m} / \mathrm{s}]\end{array}$ \\
\hline \multirow[t]{3}{*}{ Myelinated } & Large & I & $\mathrm{A} \alpha$ & $12-20$ & $72-120$ \\
\hline & Medium & II & $A \beta$ & $6-12$ & $36-72$ \\
\hline & Small & III & $A \delta$ & $1-6$ & $4-36$ \\
\hline \multicolumn{2}{|c|}{ Unmyelinated } & IV & C & $0.2-1.5$ & $0.4-2.0$ \\
\hline
\end{tabular}


Introduction

As shown in Table 1, nerve conduction velocities differ in a broad range between unmyelinated and myelinated fibers and even between myelinated nerve fibers. Unmyelinated nerve fibers are very slow in conduction velocity due to the missing of myelin sheaths; propagation of the depolarization of the membrane potential continues until it abates along the nerve fiber or reaches the axon's terminals (Kandel et al., 2012). This kind of slow information flow occurs for instance in pain perception.

On the other hand, nerve fibers myelinated by Schwann cells (c.f. Figure 6) support fast conduction of the depolarizing membrane potential. This is accomplished by formation of myelinated nonconducting areas on nerve fibers. This shape repeats over the nerve fiber until axon terminals. An action potential propagates saltatory over the nerve fiber and is always regenerated at ion channel containing nodes of Ranvier.

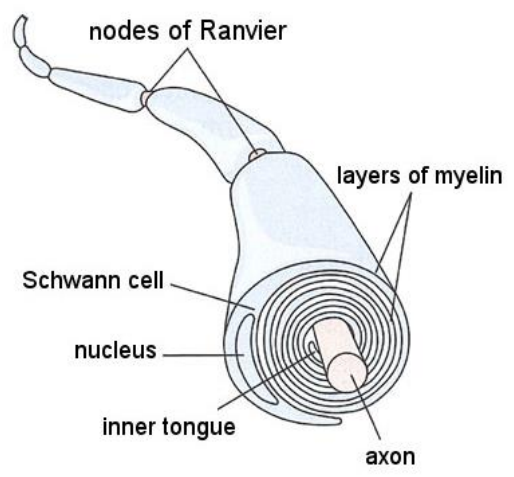

Figure 6: Myelination of an axon by Schwann cells (internode distance is about $1 \mathrm{~mm}$ ), each sheath is formed by an individual Schwann cell (by Kandel, 2012, modified).

\subsection{Physiology}

\subsubsection{Motoneuron and Motoneuron Types}

A motoneuron is described as the neuron with its cell body located in the spinal cord and its axons projecting onto effector organs (Kandel et al., 2012). The axons' terminals transmit via neurotransmitters action potentials of the motoneuron to the muscle fiber. In the case of the skeletal muscles, the neurotransmitter acetylcholine leads to a chemical cascade that causes the muscle fibers to contract, which is called neural drive. The cascade leading to muscle contraction is highlighted in chapter 2.4.6 and chapter 2.4.7.

Motoneurons can be classified as upper and lower motoneurons; the first (or upper) motoneuron projects via the corticospinal tract on spinal motoneurons. One primary motoneuron innervates 
Introduction

several secondary (or lower) motoneurons. With the principle of divergence, the signal is spread to several spinal motoneurons. On the contrary, several primary motoneurons converge on one secondary motoneuron. The principle of divergence and convergence is a fundamental principle in neural circuitries to achieve neural interplay. Polysynaptic reflex arcs enhance their information spread with interneurons massively and span a network between up to six spinal segments per interneuron (Kandel et al., 2012).

In invertebrates, the response of the muscle fiber by motoneurons can be excitatory or inhibitory, depending on the released neurotransmitter and the receptor type it binds to; on the contrary, in vertebrate's response to the binding neurotransmitter (acetylcholine) is exclusively excitatory (Schmidt et al., 2007). This means that interplay between agonist and antagonist must be accomplished by inhibition of the motoneuron itself.

\subsubsection{Motor Unit}

A motor unit (MU) is defined as a motoneuron and all by its axon terminals innervated muscle fibers (Kandel et al., 2012). Motor units are categorized in various ways. Since thresholds for a clear categorization are difficult to meet, the frame for each category is not that strict. From a physiological point of view, we can categorize motor unit types depending on differences in speed, contraction strength and fatigability, as in the following Table 2, modified from Burke et al. (Burke et al., 1974):

Table 2: Common categorization of motoneuron types according to contraction speed and fatigability (Schmidt et al., 2007, modified):

\begin{tabular}{|lll|}
\hline Name & Corresponding fiber type & Properties \\
Fast fatigue-resistant & Type $I_{\mathrm{A}}$ & Relative high force, fast twitch \\
Fast intermediate & Type $\mathrm{I}_{\mathrm{I}}$ & Between $\mathrm{I}_{\mathrm{A}}$ and $\mathrm{II}_{\mathrm{B}}$ \\
Fast fatigable & Type $\mathrm{I}_{\mathrm{B}}$ & High force, fast twitch \\
Slow & Type I & Low force, slow twitch \\
\hline
\end{tabular}

Besides this physiological and biochemical classification, also immunohistochemical and gene characterized classifications exist, but these classifications will not be discussed here in detail.

In general, fast fatigable MUs can produce relatively high forces, whereas fast fatigue-resistant MUs only cause a fraction of the force produced by fast fatigable MUs (c.f. Figure 7). Slow MUs produce a fraction of force of the fast-fatigue-resistant MUs. The fast intermediate fibers are with their properties in between $\mathrm{II}_{\mathrm{A}}$ and $\mathrm{II}_{\mathrm{B}}$ (Kandel et al., 2012; Pape et al., 2014). 
Introduction

Morphologically, two types of muscles can be discriminated: red and white muscle. Red muscles are composed of slow-twitch fibers, called type I fibers, which rely mostly on oxidative catabolism for ATP regeneration and contain high amounts of mitochondria, oxidative enzymes and myoglobin (Schmidt et al., 2007). They are surrounded by an extensive network of capillaries. They produce relative low amount of force but they are able to contract over a long period of time. This is for example of great importance to maintain erect posture over the day. On the other hand, white muscles consist of mostly fast twitch type II fibers. These fibers have different properties compared to type I fibers. The cross-bridges can produce force more effectively. They can be categorized into two different types: type $\mathbb{I}_{A}$ and type $\|_{B}$. Fast fatigable $M U s$ are called type $\|_{B}$ fibers. They are dependent on anaerobic catabolism. The fibers contain relatively large pools of glycogen to transfer ADP into ATP while glycogen is converted into lactic acid. These fibers can produce high forces in a very short period of time (brief bursts) but they need up to hours to fully recover (Kandel et al., 2012). The fast fatigue-resistant fibers of type $I_{A}$ do not fatigue that rapidly (over several minutes) and are relatively fast-twitching (Kandel et al., 2012; Pape et al., 2014). Slow-twitch muscle fibers are generally innervated by slow-twitch motoneurons, whereas fast-twitch muscle fibers are in general innervated by fast-switch motoneurons (c.f. Table 1).
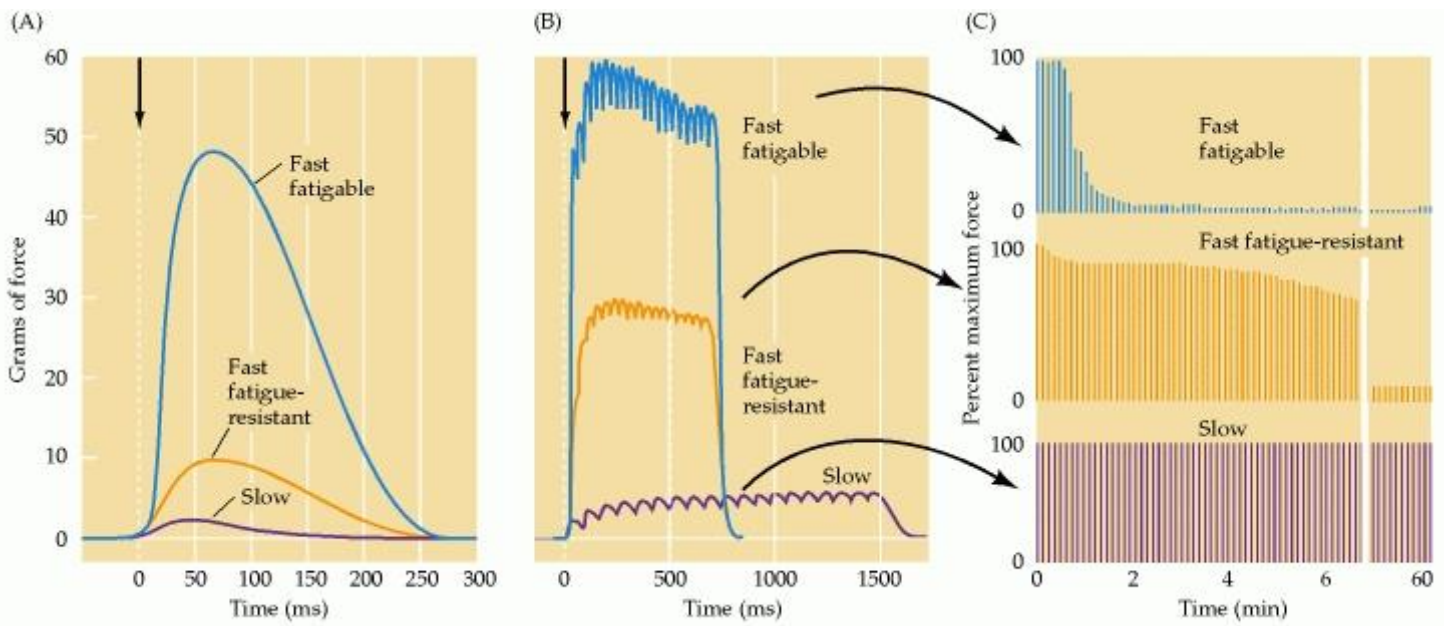

Figure 7: Comparison by Purves et al. (Purves et al., 2001) of three different motor unit types (fast fatigable [blue], fast fatigue-resistant [orange] and slow [purple]). Single motor unit responses are shown. The $\mathrm{x}$-axis shows individual time course, $y$-axis represents force development $(A, B)$ and percentage of force (C). (A) According to single motor unit action potential muscle tension changes. (B) Muscle tension in respect of repetitive subsequent stimulation. (C) Tetanic stimulation evokes maximum tension (Burke et al., 1974, modified).

\subsection{3 $\alpha$ - and $\gamma$-Motoneuron interplay}

a-Motoneurons represent about $70 \%$ of all motoneurons (Kandel et al., 2012). They are cells of the CNS which innervate skeletal muscles via neuromuscular junctions and by their action cause contraction of muscle fibers. These cholinergic neurons belong to the lower motoneurons. Cell 
Introduction

bodies are localized in the nuclei of the brainstem and the anterior horn of the spinal cord. Extrafusal muscle fibers are innervated by $\alpha$-motoneurons (Schmidt et al., 2007).

On the other hand, an actively controlled $y$-motoneuron serves as fusimotor (Pierrot-Deseilligny et al., 2000; Knikou, 2008) by controlling muscle spindle sensitivity. By actively maintaining muscle spindle length, it ensures the system possibility to work within physiological properties. $y$ Motoneurons represent about $30 \%$ of all motoneurons (Kandel et al., 2012). Their efferents innervate the intrafusal muscle fibers of muscle spindles; as well as $\alpha$-motoneurons, their cell bodies are located in the anterior horn. Axons of $\gamma$-motoneurons are smaller in diameter compared to $\alpha$ motoneurons and extinct either dynamic or static $\gamma$-fibers (Pierrot-Deseilligny et al., 2012) (c.f. Figure 8, Figure 15 and Figure 16). $I_{A}$ afferent fibers are located like a spiral in the central region of an intrafusal muscle fiber. Type II fibers are located in the adjacent. Dynamic nuclear bag fibers receive information from $I_{A}$ afferents and are innervated by dynamic $\gamma$-motoneurons, which can in turn alter $\mathrm{I}_{\mathrm{A}}$ afferents' sensitivity. By providing information about the muscles dynamic properties, dynamic $\gamma$ fibers adjust the depolarization rate of $\alpha$-motoneurons to achieve accurate contraction, especially if there is a mismatch between the intended and the achieved movement (Pierrot-Deseilligny et al., 2012). Static $\gamma$-motoneurons are part of the intrafusal muscle spindle fibers and innervate nuclear bag and nuclear chain fibers. They mainly contribute to stretch reflexes' static sensitivity. Static $\gamma^{-}$ fibers seem to provide an increase in background discharge to support active muscles (Kandel et al., 2012; Pierrot-Deseilligny et al., 2012) (c.f. Figure 15 and Figure 16).

Axons of $\gamma$-motoneurons also appear to innervate intrafusal fibers. This is useful to prevent the sensory fiber of the spindle from falling silent by keeping the central region of the spindle loaded; this phenomenon only appears during co-activation of alpha and gamma motoneurons (Kandel et al., 2012). If only $\alpha$-motoneurons are activated in an experimental setup, firing of the spindle pauses (Kandel et al., 2012). In many voluntary movements, an $\alpha-/ \gamma$-co-activation takes place. The activation of $\gamma$-motoneurons provides muscle contraction itself and, with sensing of fiber's tension, it supports optimal contraction of muscle fibers. Sensory $I_{A}$ afferent fibers build a control loop with actively controlled intrafusal muscle fibers for online control (Pierrot-Deseilligny et al., 2012). A spinal reflex can be altered by afferent fibers' presynaptic terminals, by interneurons and by $\alpha$ - or $\gamma$ motoneurons. Since involuntary postural motor control is based on reflex arcs of the spinal cord, these types of motoneurons are very important (Schmidt et al., 2007; Kandel et al., 2012; PierrotDeseilligny et al., 2012). 


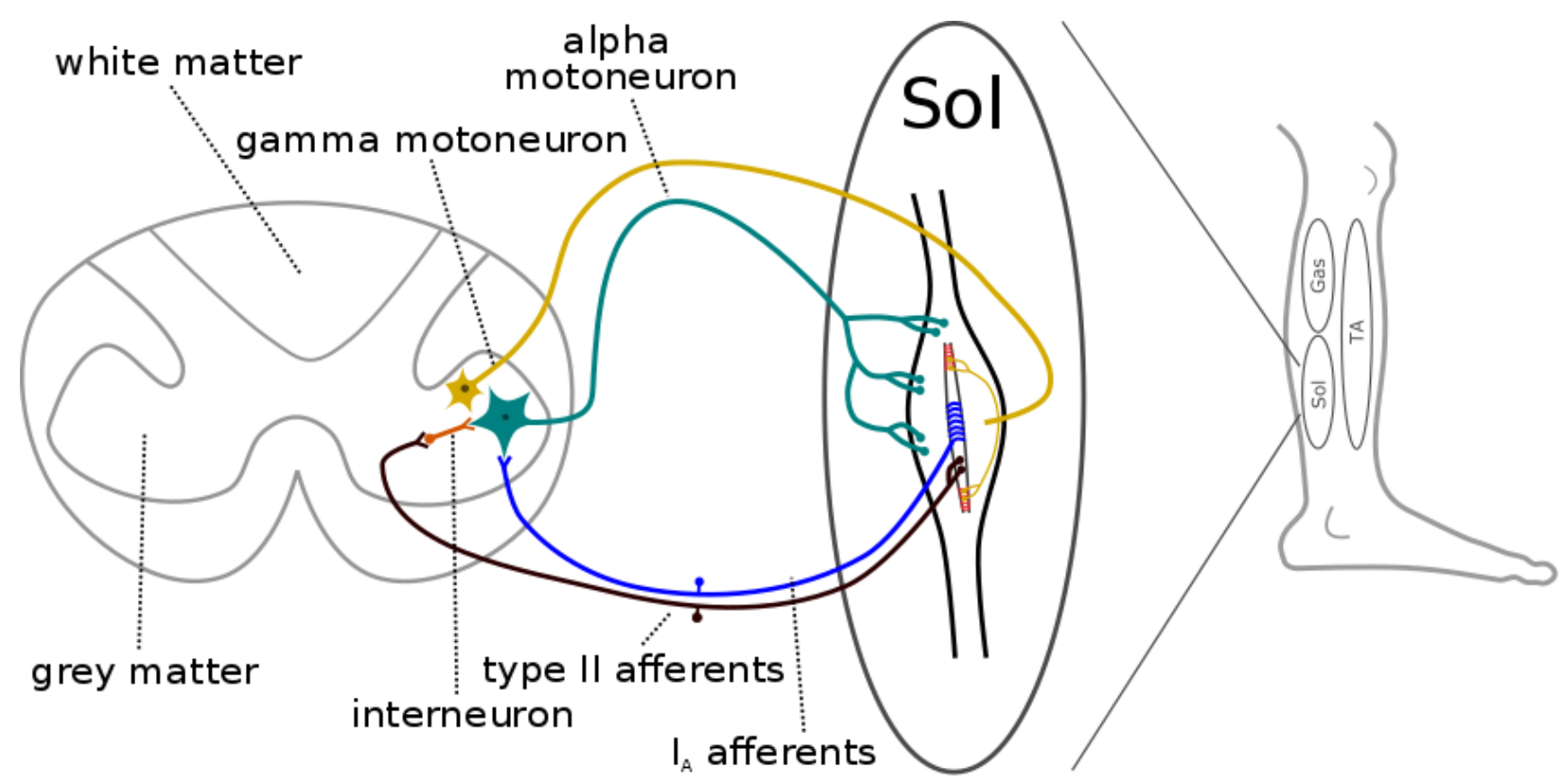

Figure 8: Neural circuitry of homonymous muscle left: cross-section of spinal cord; middle: afferent and efferent innervation of M. soleus; right: lower limb muscles (by Schmidt et al., 2007, modified).

\subsubsection{Spindle loading and fusimotor drive}

$\gamma$-Motoneurons appear as dynamic $\left(\gamma_{\mathrm{d}}\right)$ and static $\left(\nu_{\mathrm{s}}\right)$, primary and secondary endings respectively. Static $\gamma$-motoneurons possess a very low background activity in a relaxed muscle, which is not high enough to affect afferent spindle discharge or response to muscle stretch (Pierrot-Deseilligny et al., 2012). In a relaxed muscle, the response to tendon tap or muscle stretch is not altered. This gives evidence, that there is no relevant drive of dynamic MNs to resting muscles; but nevertheless it cannot be ruled out, that there may be some background activity (Pierrot-Deseilligny et al., 2012).

It can be assumed that fusimotor drive is task-dependent. In blindfolded, erect standing subjects without any support, a volley of five stimulations applied to the ipsilateral sural nerve, caused spindle activation (measured with microelectrodes in CPN) but no EMG signal was generated in the silent TA (Aniss et al., 1990). It produced a reflex response in SOL and a forward body sway. This forward sway would have unloaded the spindle endings but an activation of SOL's spindle was measured. Thus, fusimotor drive seems to support mainly moment-to-moment control of movement (Aniss et al., 1990; Pierrot-Deseilligny et al., 2012).

We know, that in contraction, muscle spindles' active endings have increased discharge, and silent endings are activated when movement is prevented (Pierrot-Deseilligny et al., 2012). This is thought to be mediated by activation of $\gamma$-motoneurons (Pierrot-Deseilligny et al., 2012). Turchick states (Pierrot-Deseilligny et al., 2012) that "the fusimotor-driven inflow from primary and secondary 
Introduction

endings during a voluntary contraction has an autogenic excitatory effect at spinal level". Gammadrive in motor learning is thought to be of great importance, and there is evidence that a greater fusimotor drive to co-contracting muscles exists, probably due to the need to perform smooth movement trajectories and for support with sensory cues; this is thought to lead to a refined voluntary drive, since spinal cord integrates those cues (Pierrot-Deseilligny et al., 2012).

Skill acquisition of new discrete motor tasks requires often co-contractions of antagonistic muscles to brace joints (Pierrot-Deseilligny et al., 2012). While an enhanced gamma drive is present in the contracting muscles, there is also evidence of enhanced fusimotor drive to co-contracting muscles (Nielsen et al., 1994; Pierrot-Deseilligny et al., 2012). The development of a new motor task also depends on information of re-afferent information and the executing lower motor centers which rely on the information. By this, gamma drive plays an important role in motor learning. Furthermore, an altered gamma drive implies neurological syndromes, for example in spasticity and gait disturbances in Parkinson's disease (Pierrot-Deseilligny et al., 2012).

$\psi$-Motoneurons contribute to learning and execution of accurate movement and are therefore of great importance when studying the potential adaptations of spinal circuitries induced by (longterm) motor training.

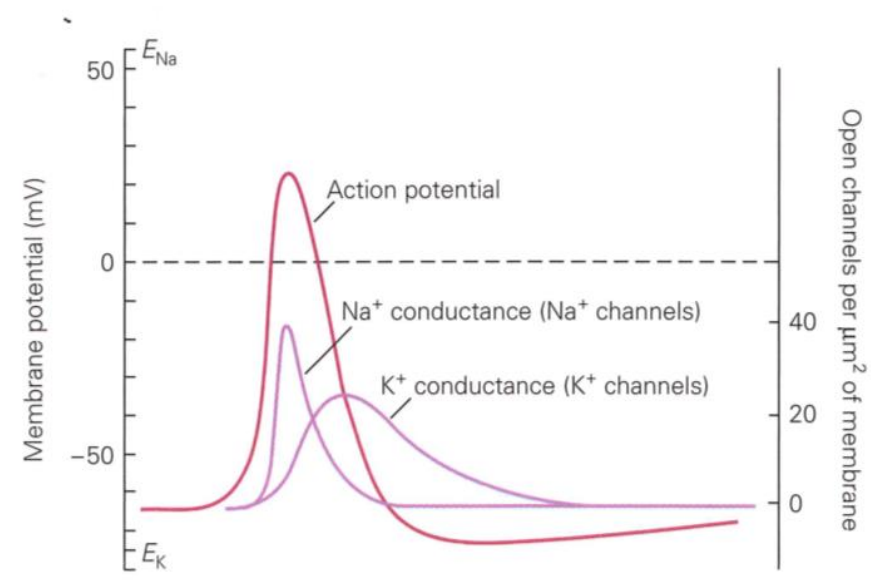

Figure 9: The figure shows generation of APs by changes in membrane-conductance for sodium and potassium, resulting depolarization and repolarization phase; achieved by patch clamp technique by Hodgkin and Huxley, 1952 (figure by Kandel et al., 2012, modified).The depolarization of the membranes potential is followed by an avalanche-like opening of sodium channels which in turn leads to opening of potassium channels, followed by repolarization according to equilibrium potentials of the involved ions.

\subsubsection{Action potential}

Action potentials are fast depolarizations of the membrane's resting potential. After depolarizing to positive potentials, driven by $\mathrm{Na}^{+}$-influx into the cell, it repolarizes fast in dependence of the cell- 
Introduction

type typical course of time to its resting potential (c.f. Figure 9). The duration of an AP is about $1 \mathrm{~ms}$ for myelinated fibers, about $10 \mathrm{~ms}$ for muscle fibers and about $200 \mathrm{~ms}$ for heart muscle cells; the duration depends on the target muscle, causing propagation along fiber and transmitter release at the axon's terminals.

The AP development starts with a very fast increase in the membrane potential. This rise lasts 0.2 $0.5 \mathrm{~ms}$ and usually zero volts are exceeded. After the peak of the depolarization phase, the repolarization of the membrane begins and the cell returns to its resting potential. Some types of cells possess after-polarizations that can be depolarizing (muscle) and hyperpolarizing (spinal). As described below and depicted in Figure 10, cells possess after their excitation above threshold and generation of an AP an absolute and a relative refractory period. These time intervals hinge on gated ion-channels and are cell-specific.

Depending on the all-or-none-law, APs are only generated if a cell-specific threshold is exceeded, which is usually a depolarization from its resting potential up to $-50 \mathrm{mV}$ (Cole et al., 1939). By patch clamp techniques the membranes resting potential and following processes are determined (Hodgkin et al., 1952). The excitation of the membrane lasts mostly less than $1 \mathrm{~ms}$ and the depolarization phase itself actuates processes which in turn lead to repolarization. The course of APs with regard to the cell's excitability - which is a crucial property of muscle and nerve cells - is cellspecific and time courses are very stable. Membrane properties that lead to an elicited AP can be described as follows: the equilibrium potential of $\mathrm{K}^{+}$ions is mainly responsible for the membrane's resting potential since conductivity is highest for potassium at rest; $\mathrm{Na}^{+}$-influx is the driving force of the depolarization of the membrane potential due to voltage-gated sodium channels that open in the depolarization phase avalanche-like; the conductivity for sodium raises volatile and is dependent on the amount of initial depolarization (Kandel et al., 2012).

In the repolarization phase, $\mathrm{K}^{+}$-channels contribute to the AP when membrane potential is repolarizing to membrane's resting potential. The equilibrium potential for potassium is about $100 \mathrm{mV}$, resulting in $\mathrm{K}^{+}$-efflux between $-60 \mathrm{mV}$ and $+60 \mathrm{mV}$. This means that opening of potassium channels already begins in the depolarization phase, but sodium conductivity veneers the effect of potassium in this phase (Kandel et al., 2012) (c.f. Figure 9).

Due to ion-channel specific properties, cells can generate series of APs depending on the relative refractory period. A long-lasting excitation for example leads to an inhibition of new APs, whereas cells that possess, due to drugs or disease, more positive resting membrane potential than $-50 \mathrm{mV}$ up to $-60 \mathrm{mV}$, are not excitable (Schmidt et al., 2007). 


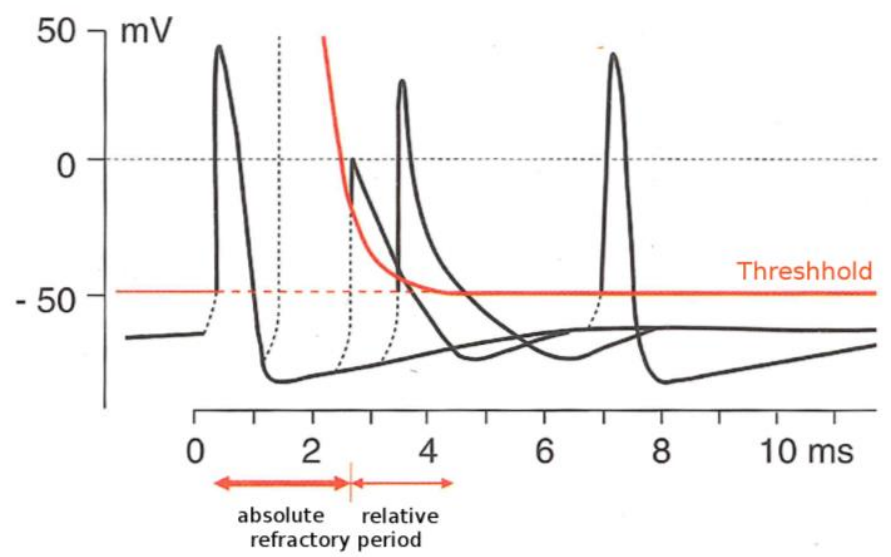

Figure 10: For a short period of time depolarizations of the axon cannot elicit a new AP. After $\sim 2.5 \mathrm{~ms}$ depolarizations far above threshold can elicit a new smaller AP, whereas after $\sim 4.5 \mathrm{~ms}$ an AP with similar size like the first developed AP can be generated (by Schmidt et al., 2007, modified).

Depending on the all-or-none law muscle fiber contractions appear in a rate-coded manner. Slow frequency causes single muscle fiber contractions, whereas, with increasing frequency, fibers respond with multiple contractions until tetanic contraction. In this sense, we speak of a frequency or rate coding of information. The rate in which a motor unit discharges and the number of motor units recruited determine the amount of exerted force (Duchateau et al., 2014).

It is important to understand the mechanism of information coding and how electrical signals propagate along different fibers. The organism accomplishes, by sensing of its position in space and intrinsic properties (upward information flow), the optimal adjustment according to the actual circumstances. By driving motoneurons and finally recruiting muscle fibers (downward information flow), to achieve a proper position, the interplay between up- and downward information flow becomes important (Kandel et al., 2012).

\subsubsection{Neuromuscular junction and propagation}

As neuromuscular junction, the formed structure by a chemical axon terminal of a motor unit and the adjoining muscle fiber is meant. The axons' terminal builds up a structured shape with the muscle fibers' membrane. The emerging synaptic cleft in between has a diameter of $10-50 \mathrm{~nm}$ (c.f. Figure 11). Surface extension is present on pre- (invagination) and postsynaptic side (basal lamina).

Presynaptically the neurotransmitter acetylcholine is released, which in turn binds on the postsynaptic membrane. On the presynaptic side of the synapse, an incoming AP leads to an opening of voltage-gated $\mathrm{Ca}^{2+}$-channels; this causes a migration of synaptic ACh-containing vesicles to the axons' terminals. After docking to the terminals, exocytosis causes the release of ACh into the 
Introduction

synaptic cleft. ACh diffuses to the postsynaptic side, binds, and opens ionotropic, receptor-gated channels which are permeable for $\mathrm{Na}^{+}, \mathrm{K}^{+}$and $\mathrm{Ca}^{2+}$. Acetylcholinesterase splits $\mathrm{ACh}$ into choline (which is presynaptic resumed) and acetate (which passively diffuses out of the synaptic cleft). The rate-coded transmitter-release at the neuromuscular junction translates incoming information of the nerve fiber into muscle contraction; this is called electrochemical transduction. By this, in electromyography techniques, only currents that occur in fibers can be analyzed, but no direct measurement of motoneuron behavior is possible. Detection of motoneuron currents involves invasive techniques (Schmidt et al., 2007).

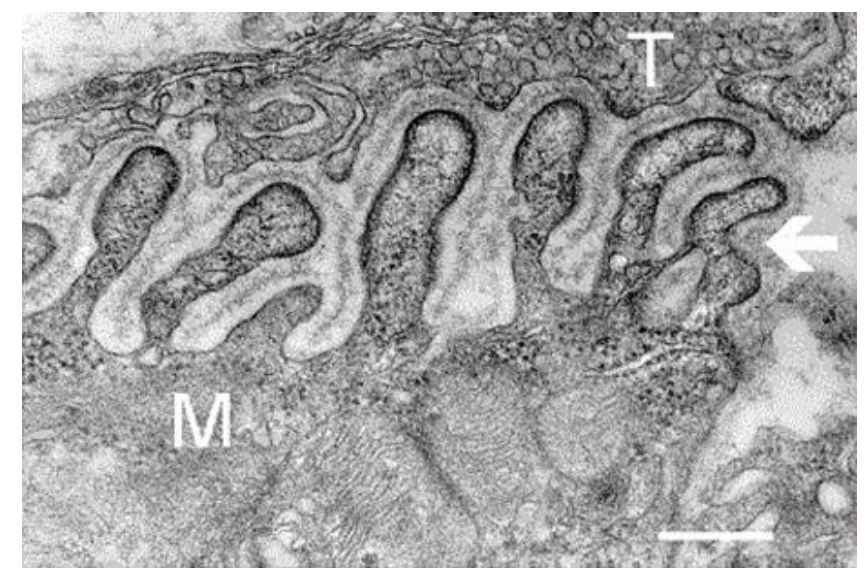

Figure 11: Cross section of neuromuscular junction, $\mathrm{T}$ : axon terminals, M: muscle fiber, arrow: junctions with basal lamina scale: $0.3 \mu \mathrm{m}$ (by Wikimedia Commons, originally uploaded to en.wikipedia on $10^{\text {th }}$ March 2006; originally source: Synapse Web at the National Institute of Mental Health, National Institutes of Health, accessed $1^{\text {st }}$ June 2018).

The resulting postsynaptic current is mainly carried by $\mathrm{Na}^{+}$- and $\mathrm{K}^{+}$-ions. This sequent depolarization from the post-synaptic side's resting membrane potential is called endplate potential (EPP). Conductance of ACh-gated ion channels is very variable for different muscle fiber types. For Example, white fibers possess a high conductance for cations compared to red fibers. In humans, the EPP of excitatory synapses represents a depolarization of the muscle fiber. The resulting membrane potential from an excitatory synapse spreads out passively over the muscle fiber's membrane (sarcolemma) (c.f. Figure 12). If the receptor potential exceeds the muscle fibers threshold potential, $\mathrm{Na}^{+}$-channels cause the generation of a muscle AP. Sequent, the AP spreads over the membrane, enters the transverse tubules and activates $\mathrm{Ca}^{2+}$-sensitive dihydropyridin and ryanodine receptors inside of the muscle fiber. $\mathrm{Ca}^{2+}$ is released from the sarcoplasmic reticulum into the cytosol. Calcium initiates thereby the contraction of myofibrils in the cross-bridge cycle (Schmidt et al., 2007). 
Introduction

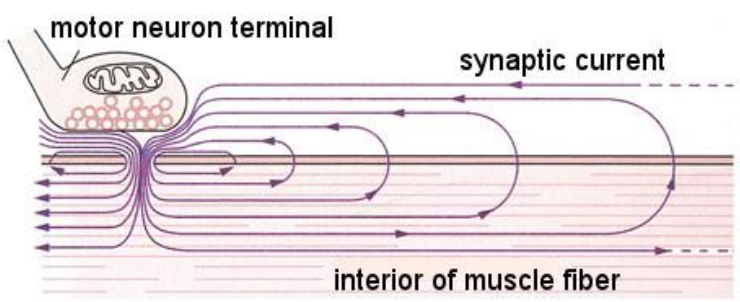

Figure 12: Elicited synaptic current caused by transmitter release of motor neuron terminals decays along the muscle fiber. Current propagates in the interior of the muscle fiber and since the electric circuit must be closed, it flows back in the exterior of the muscle fiber (by Kandel et al., 2012, modified).

This further opening of voltage-gated $\mathrm{Ca}^{2+}$-channels of the transversal tubule system, and the activation of the ryanodine system, lead to the release of $\mathrm{Ca}^{2+}$ from the sarcoplasmatic reticulum, resulting in a fast and massive increase in intracellular concentration of $\mathrm{Ca}^{2+}$ that finally induces muscle contraction. This coupling of transmitter ( $\mathrm{ACh}$ ) binding and the stepwise release of $\mathrm{Ca}^{2+}$, which leads to muscle contraction, is called electro-mechanical coupling (Schmidt et al., 2007).

For the generation of higher contraction force, more muscle fibers must be recruited (Kandel et al., 2012). In order to recruit more muscle fibers, neural drive increases, compared to low forces, resulting in more APs per time, that appear on a muscle at once, elicited by the activation of more motoneurons (Kandel et al., 2012). These APs propagate over the muscle and are detectable. With these techniques, also the mapping of muscle activity in terms of its spatial orientation and time is possible.

\subsubsection{Emergence of force}

The cross-bridge cycle is the accepted model to explain repetitive muscle activation as well as resulting phenomena like fatigue. The underlying accepted model of muscle contraction itself is the sliding filament theory (Huxley et al., 1954 a; b). It can be concluded that thick myosin filaments possess 'heads' which bind under the influence of $\mathrm{Ca}^{2+}$ to thin actin filaments. Myosin filaments are at their basis attached to titin filaments which are, as well as actin filaments, anchored with Z-discs. By a change in conformation, i.e. by execution of a stroke of the myosin's head lever arm, shortening of the sarcomere is achieved and turns into the emergence of force. Subsequently binding of ATP leads to return to resting state. While the muscle is at rest, actin- and myosin filaments possess an ideal overlap to produce force via shortening. This contraction itself is effect of the electrical stimulus evoked at the neuromuscular junction and the sequent release of $\mathrm{Ca}^{2+}$ (Schmidt et al., 2007). 
Introduction

The Actomysoin ATPase cycle (Goldman, 1987; Fitts, 2007; Webb et al., 2011; Kandel et al., 2012) tries to complete the above theory by explaining more detailed the change in configuration of myosins' heads.

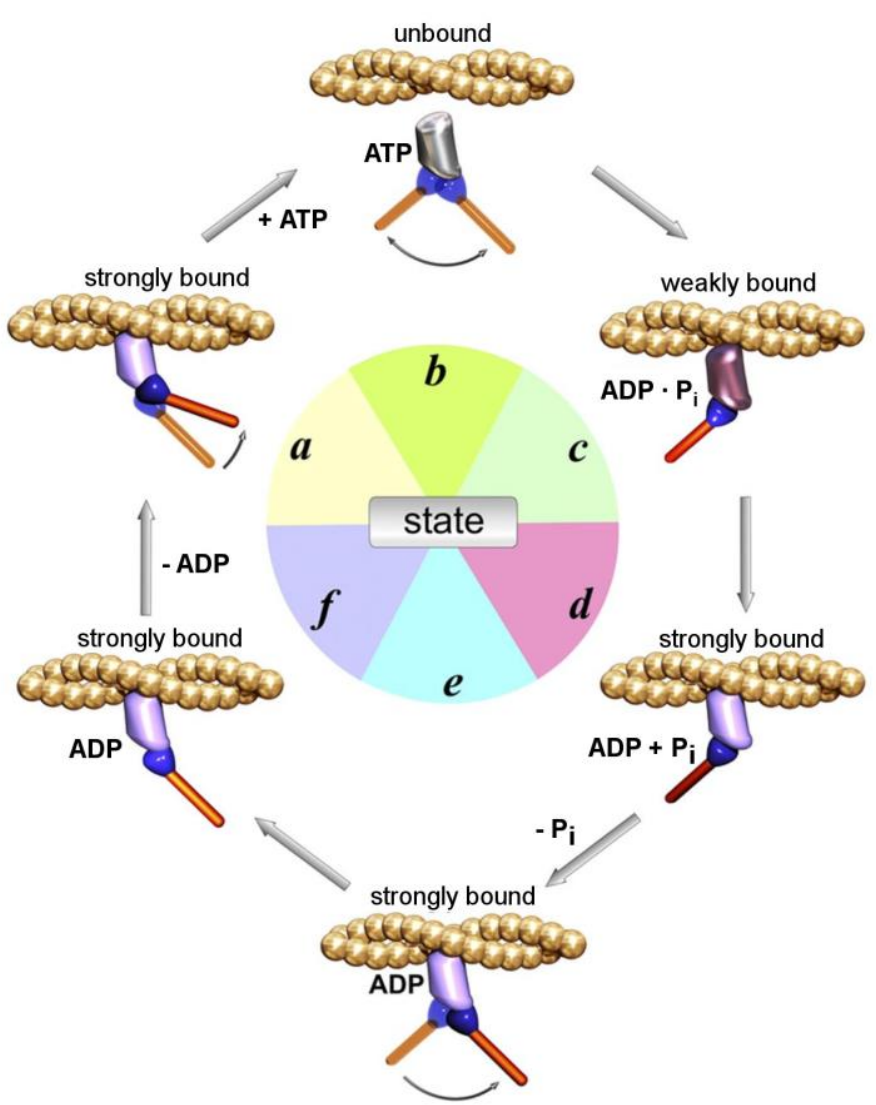

Figure 13: Actomyosin ATPase cycle (by Fitts 2008, modified). Different configurational states (a - f) of myosin heads and corresponding configurations of the lever arm are shown. Motor domain is colored in metallic gray (free form), purple (weakly bound) and violet (strongly bound); converter shown in blue, lever arm in orange. Actin monomers are shown as golden spheres.

As depicted in Figure 13, different configurational states are described by consecutive letters (a-f):

- a: myosin is strongly bound (rigor complex): actomyosin $\bullet$ myosin

- b: by binding of ATP and dissociation of the complex, the lever arm is rotated to the prepower stroke position

- c: hydrolysis follows leading to binding of myosin $\bullet A D P \bullet P_{i}$ weakly to actin

- $d$ : followed by strongly binding

- e: by binding to actin, $\mathrm{P}_{\mathrm{i}}$ dissociates and causes the power stroke

- $f$ : the swing of the tail completes and lead to the rigor complex (a) 
Introduction

These two underlying principles of muscle contraction are of great importance to understand intrinsic muscle properties and the appearance of fatigue, and why we are able to classify muscle fibers with regard to their physiological and intrinsic properties. 
Introduction

\subsection{Motor Control}

\subsubsection{Hierarchy of motor control}

Processing of sensory afferent input and efferent motor commands is organized in areas that are hierarchically interconnected. These areas are spinal cord, brainstem and forebrain. Motor tasks as reflexes, rhythmic as well as voluntary movements, are controlled by these areas. Neural circuitries are capable of regulating complex motor responses (Kandel et al., 2012).

In the following, a summary of Kandel's description of hierarchy of motor control is outlined (Kandel et al., 2012): Movement related sensory information is processed simultaneously in different hierarchically organized structures and systems. Neural circuits located in the spinal cord control reflex mediation and rhythmic circuits. In contrast, reflexes of face and mouth are controlled by systems located in the brainstem. Interneurons carry out transmission of information to other nerve cells. The simplest reflex arc is the monosynaptic reflex arc. The monosynaptic stretch reflex, for instance, is mediated without any interneuron and displays a control loop of the homonymous muscle. The spinal cord appears, due to its structure and function, as lowest in hierarchy. Higher centers of the brain provide downstream flow of information to facilitate interneurons and/or motoneurons and thereby refine movement patterns. This leads to facilitation or inhibition of reflexes and is present in smooth muscles' interplay. Bending of a joint is accomplished by a control loop that leads to facilitation of the flexor muscle and inhibition of the extensor muscle. Medial and lateral brainstem neurons project processed information from the cerebral cortex, the cerebellum and subcortical nuclei to the spinal cord. Two descending systems are present: the medial descending system that integrates somatosensory, visual and vestibular information to refine posture control; and the lateral descending system that regulates goal-directed movements of more distal limb muscles. The highest level of motor control, with regard to neural representation, is present in the cortex; it is structured into the primary motor cortex with its MNs projecting directly to secondary MNs of the spinal cord via the Tractus corticospinalis. Several premotor areas contribute to motor control as well; they contribute to planning of complex motor sequences and information from the posterior parietal and prefrontal association cortices are integrated.

Besides this, two other parts of the brain alter planning and execution of the performed task: basal ganglia and cerebellum. They are capable to alter cortical and brainstem motor areas by feedback circuitries. This process is achieved by involvement of thalamic structures. Loop circuits flow through different regions of the thalamus. Basal ganglia access information of the cortex and project, after integration of information, back to the cortex, building open- and closed-loop control systems (c.f. chapter 2.5.2). 
Introduction

It is known that cerebellum and basal ganglia regulate and refine posture and smooth movements, i.e. muscle interplay. Parkinson's and Huntington's disease, both degenerative disorders of the basal ganglia, result in anomalous posture and involuntary movements (Kandel et al., 2012). The function of basal ganglia appears as cognitive processing of adaptive motor planning and motivation of motor tasks, whereas damage of the cerebellum results in discoordination and loss of accuracy for especially limb motor tasks. Neural circuits located in the cerebellum are responsible for timing, coordination and learning of motor tasks (Kandel et al., 2012).

\subsubsection{Open and closed loop systems}

Motor control of movements is achieved by control and support of motor systems. Early examinations led to the assumption that motor behavior is controlled in an open loop (feedforward) system. This theory describes that movement representations are saved as motor programs in the brain and can be loaded by need (Kandel et al., 2012). By this, nerves are driven by the primary motor cortex without any possibility to interfere and refine movements while they are performed. Alteration of joint trajectories and overall task-performance can only be achieved after execution of the task for subsequent task iterations. This approach evolved as common opinion since ballistic movements are performed too fast to for any intervention or refinement process while the task is performed; optimization of movements can only be accomplished after completion of the movement task (Ehrlenspiel et al., 2010).

On the other hand, the theory of a closed loop (feedback) system was developed. Within this theory it is possible to study the state of different intrinsic parameters and properties to adjust neural drive while the movement is performed. This theory describes the control of slow movement like reaching tasks (Ehrlenspiel et al., 2010).

Since both, open and closed loop control, need time to measure, process, integrate and drive nerves, and implement thus refinements in the motor task, the time takes too long for a real-time (online) measuring process without any measurable latency. The actual approach is finding and defining neural structures that are capable to provide the possibility to measure online.

Usually, for the performance of a motor task, entire programs of motor behavior are loaded and executed. These programs rarely drive single muscles. Mostly their motor programs lead to the execution of a movement accomplished by the activation of several synergistic, interrelated groups of muscles. It can be considered that muscle synergies are supporting fine muscle interplay and that 
Introduction

these facilitate the execution of learned and saved motor patterns (Ting et al., 2007; Chvatal et al., 2011, 2013; Oliveira et al., 2012; Pierrot-Deseilligny et al., 2012; Sawers et al., 2015).

\subsubsection{Feed-forward inhibition and feedback inhibition}

To achieve fine muscle interplay between synergistic and antagonistic muscles, there must be a way to inhibit muscles from being activated. Since the underlying control mechanism in human is achieved exclusively by excitatory nerve fibers (Schmidt et al., 2007), two ways of inhibition must be explained (c.f. Figure 14).

The feed-forward inhibition of a flexor muscle is achieved by projection from extensor muscle's afferent fibers onto inhibitory interneurons, leading to inhibition of efferent fibers of the flexor muscle. Vice versa, feedback inhibition of motoneurons is achieved by a control loop of the extensor muscle itself containing inhibitory interneurons.

\section{Feed-forward inhibition}

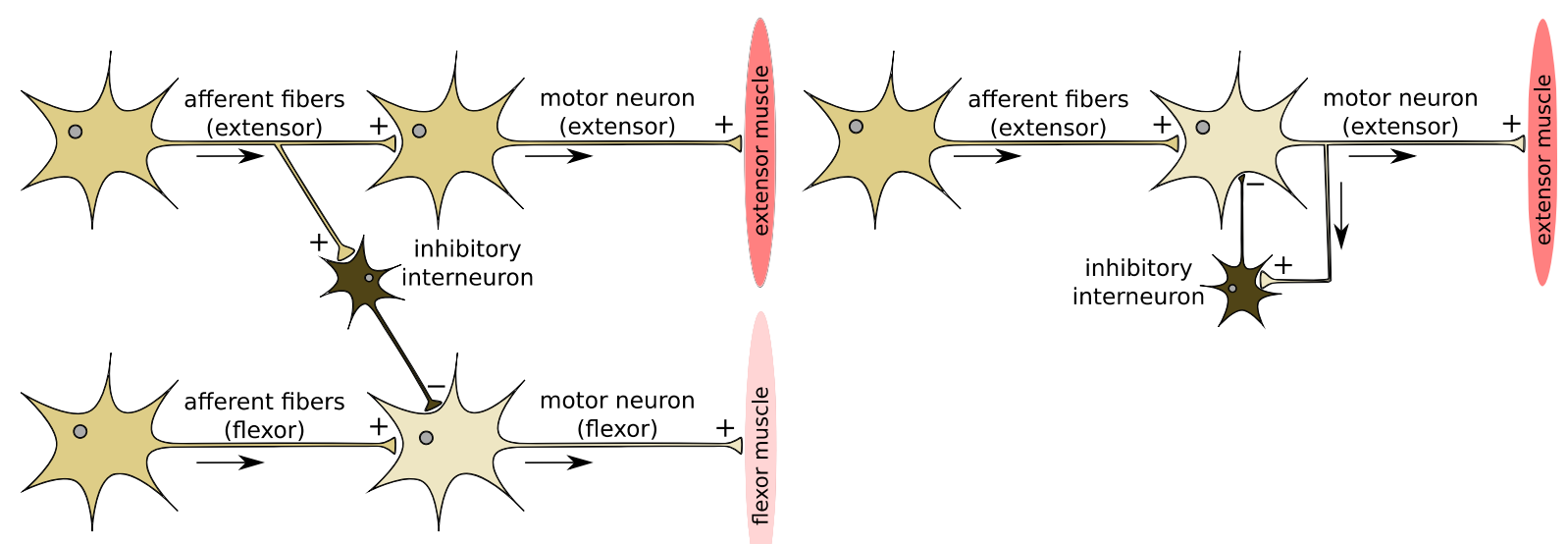

Figure 14: Feed-forward inhibition (left) and feedback inhibition (right) (by Kandel et al., 2012, modified). Muscle depicted reddish, cells yellowish; axon activity in dark yellow shows high activity, light yellow shows less activity achieved by inhibition.

\subsubsection{Neurons' sensory and proprioceptive feedback system}

Neurons can be classified as unipolar, bipolar, or multipolar neurons; depending on growth of axons and dendrites. The sensory system provides the possibility to learn by vision, hearing, feeling, tasting, smelling and pain (classical classification) and by temperature, itch, proprioception and vestibular sense of balance (modern classification) (Kandel et al., 2012). Input of cells, receiving a stimulus, is described in three steps: (1) the physical stimulus itself, (2) transformation of the stimulus into electrical event and (3) the response to the sensation which can be seen as perception 
Introduction

of the stimulus. Perceptions are constructions of our nervous system and our brain, depending on the sum of our experiences. Research suggests evidence of a complex repertoire of neural representations beginning with birth. This stock is highly modifiable from birth to age (Lohmann et al., 2014).

The basis of all sensory systems is identical in their organization between the single systems. By stimulation, all systems convey information about modality, location, intensity and timing of a stimulus; the combination and integration of these four components causes sensation (Kandel et al., 2012).

Somatosensory neurons, located in the dorsal root ganglia of spinal nerves, outline efferent nerve fibers, i.e. axons of the bipolar cells, towards the CNS. One neuron receives its input from several afferent fibers that send proprioceptive and nociceptive information towards the CNS. Thereby, these neurons process and select proprioceptive information before transmitting them to the CNS (Schiebler et al., 2007). This is called upstream of information, as mentioned above. Sensory cells appear in different types, depending on the stimulus they are designed to receive; whereas cells sensing input information are present for various types of stimuli (Kandel et al., 2012). A summary of these types of receptors active in somatic sensation, and their innervation and functionality, is shown in Table 3.

Depending on the quality of the stimulus, this causes excitation or inhibition of the sensory neuron, depending on time and spatial changes in membrane potential. Integration of this information is carried out by neurons and is achieved by higher centers of the brain. In the sensory system, the reaction to a stimulus is the cell's depolarization; in contrast, rod cells in the human eye react to a stimulus with hyperpolarization. Graded receptor potentials are primarily generated by sensory input (Schmidt et al., 2007).

Two diverse mechanisms exist for the coordination of reflex pathways: convergence and divergence. Divergence leads to enhanced sensory inputs in reflex pathways and is responsible for coordination of muscle response; divergent distribution of information $I_{A}$ afferents appears in the spinal cord to the homonymous muscle even to synergistic muscles (Kandel et al., 2012). As stated by Kandel (Kandel et al., 2012): “[...] stretch reflex pathways provide a principal mechanism by which contractions of different muscles can be linked together in voluntary as well as reflex actions" which makes neurophysiological investigations on this level to the optimal target area to research spinal plasticity via stretch reflex measurements. 
Table 3: Receptor types active in somatic sensation (by Kandel et al., 2012, modified):

\begin{tabular}{|c|c|c|c|}
\hline Receptor type & Fiber group & $\begin{array}{l}\text { Fiber } \\
\text { name }\end{array}$ & Modality \\
\hline \multicolumn{3}{|c|}{ Cutaneous and subcutaneous mechanoreceptors } & Touch \\
\hline Meissner's corpuscle & $A \alpha, \beta$ & RA & Stroking, fluttering \\
\hline Merkel disc receptor & $A \alpha, \beta$ & SAI & Pressure, texture \\
\hline Pacinian corpuscle & $A \alpha, \beta$ & PC & Vibration \\
\hline Ruffini ending & $A \alpha, \beta$ & SAII & Skin stretch \\
\hline Hair-tylotrich, hair-guard & $A \alpha, \beta$ & $\mathrm{G} 1, \mathrm{G} 2$ & Stroking, fluttering \\
\hline Hair-down & & $\mathrm{D}$ & Light stroking \\
\hline Field & $A \alpha, \beta$ & $\mathrm{F}$ & Skin stretch \\
\hline \multicolumn{3}{|l|}{ Thermal receptors } & Temperature \\
\hline Cool receptors & A $\delta$ & III & Skin cooling $\left(25^{\circ} \mathrm{C}\right)$ \\
\hline Warm receptors & C & IV & Skin warming $\left(41^{\circ} \mathrm{C}\right)$ \\
\hline Heat nociceptors & $A \delta$ & III & Hot temperatures $\left(>45^{\circ} \mathrm{C}\right)$ \\
\hline Cold nociceptors & $\mathrm{C}$ & IV & Cold temperatures $\left(<5^{\circ} \mathrm{C}\right)$ \\
\hline \multicolumn{4}{|l|}{ Nociceptors } \\
\hline Mechanical & $A \delta$ & III & Sharp, pricking pain \\
\hline Thermal-mechanical & $A \delta$ & III & Burning pain \\
\hline Thermal-mechanical & $\mathrm{C}$ & IV & Freezing pain \\
\hline Polymodal & $\mathrm{C}$ & IV & Slow, burning pain \\
\hline \multicolumn{4}{|l|}{ Muscle and skeletal mechanoceptors } \\
\hline Muscle spindle primary & $A \alpha$ & la & Muscle length and speed \\
\hline Muscle spindle secondary & $A \beta$ & II & Muscle stretch \\
\hline Golgi tendon organ & $A \alpha$ & Ib & Muscle contraction \\
\hline Joint capsule & $A \beta$ & II & Joint angle \\
\hline \multicolumn{4}{|l|}{ mechanoceptors } \\
\hline $\begin{array}{l}\text { Stretch-sensitive free } \\
\text { endings }\end{array}$ & & III & Excess stretch or force \\
\hline
\end{tabular}

In contrast, convergent input of interneurons enhances the versatility of reflex responses. This strategy can be optimally described by the example of muscle's $I_{B}$ afferents, which receive input from Golgi tendon organs. Stimulation of these fibers leads to autogenic inhibition (inhibition of homonymous motoneurons) by bi- or trisynaptic inhibition (Kandel et al., 2012). The interneurons' $I_{B}$ afferents are projecting to the CNS to form synapses with muscle spindle $\mathrm{I}_{\mathrm{A}}$ afferents, diverse cutaneous receptors and afferent fibers from joints, plus they receive excitatory and inhibitory input from different descending pathways (Kandel et al., 2012). The connections of the spinal cord with the axons of tendon organs are thought to participate in the spinal reflex network coordinating lower limb motor tasks (Kandel et al., 2012). Centrally generated descending motor commands alter properties of spinal reflex pathways by modifying their properties. 
Introduction

\subsubsection{Muscle spindle and its role in motor control}

Muscle spindles' afferents represent muscles' sensory fibers and are named by their shape. They are small intramuscular encapsulated sensory receptors which sense changes in length of its connected muscle fibers and provide therefore proprioceptive feedback information for the CNS; this system is elementary necessary to maintain posture and also to react adequately to external and internal stimuli (Kandel et al., 2012).

As depicted in Figure 15 and Figure 16, muscle spindle afferents project with der fibers towards the CNS. By this, depending on the necessity, how fast information must be received, fibers are mainly myelinated. For our investigation of neural drive of lower limb muscles, Figure 15 and Figure 16 describe important structures to understand the physiological background.

A muscle spindle consists of three main components: (1) intrafusal muscle fibers with noncontractile endings, (2) myelinated sensory endings that appear in large diameter originating from the central region of the intrafusal fibers and (3) myelinated motor endings that appear small in diameter and innervate the polar, contractile region of intrafusal fibers. Muscle spindles are arranged in parallel to the fibers of the skeletal muscle, and if skeletal muscle fibers and intrafusal fibers are stretched (i.e. "spindle loaded"), sensory endings are stretched as well, resulting in an increased firing rate. If the muscle changes its length, intrafusal muscle fibers change in length as well. Due to this, the activity of sensory endings increases if the muscle is stretched. On the other hand, a shortened muscle leads to decreased activity of sensory endings. Gamma motoneurons innervate intrafusal fibers, whereas large-diameter alpha motoneurons innervate extrafusal muscle fibers (Kandel et al., 2012). The behavior and properties of these motoneurons are explained in chapter 2.4.3.

Figure 16 depicts a muscle spindle located for example in the muscles of the lower limb. Afferent fibers are colored in blue (type $I_{A}$ fibers) and red (type II fibers), efferent fibers in yellow (axon of ${ }^{-}$motoneuron). Intrafusal muscle fibers contain dynamic $\gamma$-fibers at their end poles and are wrapped by $I_{A}$ afferents. Static nuclear bag fibers are wrapped by type II fibers and $I_{A}$ fibers as well. Furthermore, static nuclear bag fibers possess static $\gamma$-fibers. Both fibers are grouped side by side and are surrounded by the capsule. Monosynaptic stretch reflex is a suitable physiological pathway to explain the underlying concept (c.f. Figure 15). If the muscle is stretched (for instance by a stimulus on the stretched knee tendon) $\mathrm{I}_{\mathrm{A}}$ and II afferent fibers are projecting proprioceptive feedback towards $\gamma$-motoneurons, arising an AP on the homonymous muscle. Interneurons in the central pathway provide the system possibility to receive further input from antagonistic muscles or higher cortical areas. Higher cortical areas can project on $\alpha$ - and $\psi$-motoneurons as well. After afferent information is processed, the muscoskeletal system must be driven to enable motor 
behavior. This is accomplished by the efferent system. In the PNS efferent fibers possess axons, originating $\alpha$-motoneurons of the spinal cord, which project over large distances from the CNS toward the peripheral effector organs (Schmidt et al., 2007; Kandel et al., 2012).

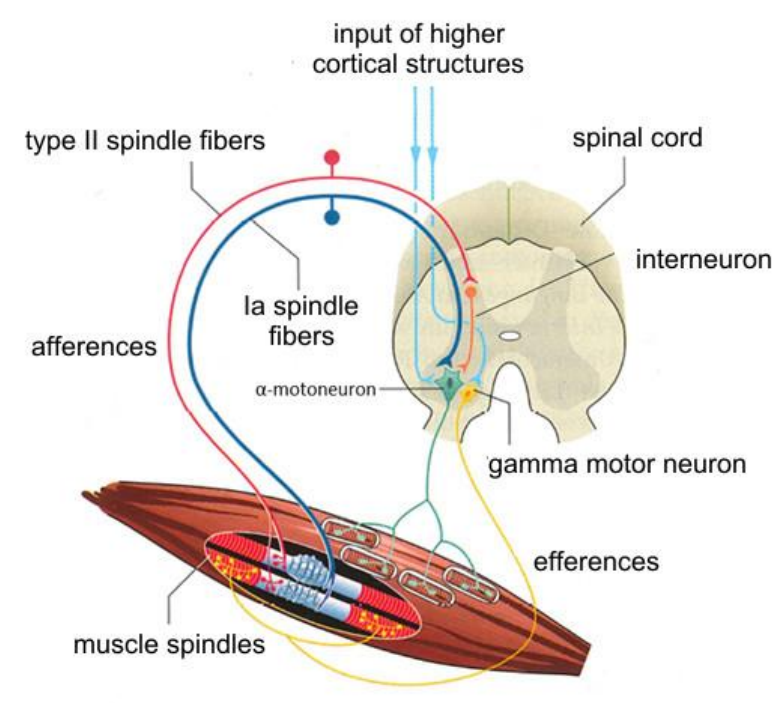

Figure 15: Depiction of a control loop with feedback inhibition showing cortical drive and circuitry in spinal cord level (by Schmidt et al., 2007, modified).

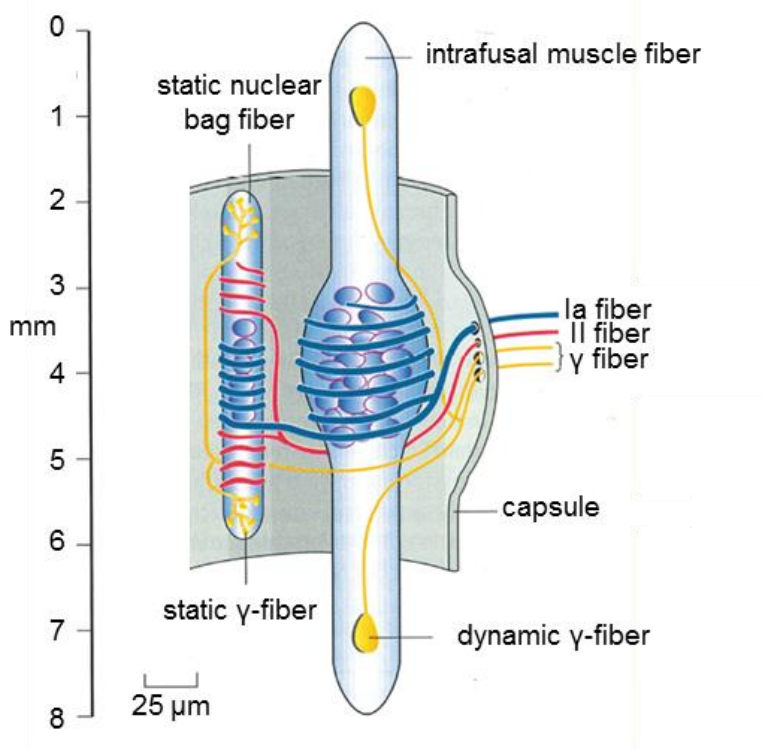

Figure 16: Structural design of muscle spindles (by Schmidt et al., 2007, modified).

Type I and type II fibers differ in properties and their physiological purpose; types refer to primary (I) and secondary (II) endings of the afferent nerve fibers. Type I sensory fibers are divided into type $I_{A}$ and type $I_{B}$. Type $I_{A}$ fibers sense the rate how fast a muscle stretch changes by sensing changes in muscle length and velocity, whereas type $I_{B}$ fibers (Golgi tendon organ) sense changes in muscle tension. Type II sensory fibers are highly myelinated, provide very fast transmission of information and do not adapt. They are connected to nuclear chain and bag fibers.

The $y$-motoneuron serves as an actively controlling fusimotor, which controls muscle spindle sensitivity. Static fibers of $\gamma$-motoneurons innervate nuclear bag and nuclear chain fibers. Static gamma motoneurons increase firing of muscle spindles; dynamic fibers of $\gamma$-motoneurons are capable of enhancement through $\mathrm{I}_{\mathrm{A}}$ afferent fibers. We can conclude that $\alpha$-motoneurons represent a type of efferent fibers that innervate muscle fibers at the neuromuscular junction. On the contrary $\gamma$-motoneurons are actively controlled sensing elements that contribute to motor control (Kandel et al., 2012).

Investigation of $I_{A}$ muscle spindle afferents is of great importance for us since it is a good tool to research spinal plasticity due to long-term motor skill training (Capaday et al., 1990; Nielsen et al., 
Introduction

1993 b; Adkins et al., 2006; Perez et al., 2007; Knikou, 2008; Ogawa et al., 2009; Thompson et al., 2009; Pierrot-Deseilligny et al., 2012). Research of $I_{A}$ afferent fibers is purposeful, since these lowest neural circuitries are affected by long-term motor skill training as well as motor programs of higher neural structures.

\subsubsection{Reflexes}

Reflexes depict fast involuntary changes of the muscle's state as a reaction to a perceived stimulus (Kandel et al., 2012). A stimulus is transmitted from the periphery via the peripheral nervous systems to the spinal cord. Afferents project directly on efferents or interneurons (c.f. Figure 15). Depending on the involvement of interneurons in the reflex arc, reflexes can be distinguished as monosynaptic (direct projection on $\alpha$-motoneurons) or polysynaptic (interneurons involved).

The tendon-jerk reflex is an example for a proprioceptive muscle reflex where the monosynaptic reflex arc affects the homonymous muscle. Sherrington was the first scientist who stated that a muscle contraction is accomplished by relaxation of the antagonistic muscle in the year of 1897 (Enoka, 2008) and called the behavior "reciprocal inhibition" (Pierrot-Deseilligny et al., 2012). A reciprocal inhibition of agonist muscles involving monosynaptic reflex testing was first designed by Lloyd in the year 1941 and published later on with colleagues (Laporte et al., 1952). It led to the assumption that due to the very short latency of the reflex, the circuitry is designed monosynaptic (Laporte et al., 1952; Pierrot-Deseilligny et al., 2012). Later, intracellular recordings showed that one interneuron is involved in the la inhibitory pathway (Eccles et al., 1956).

As depicted in Figure 15, $I_{A}$ afferent fibers directly project onto $\alpha$-motoneurons of the homonymous muscle. Excitation or experimental stimulation of afferents causes neural drive to the homonymous muscle (which in turn causes contraction). Experimental standardization for research of H-reflex (Hoffmann, 1910) is achieved by an adjustment of electrical stimulation intensity, build on Henneman's postulation regarding the size principle of motoneurons according to their excitability (Henneman et al., 1965). For this purpose $H_{\max } / M_{\max }$ tuning curves are recorded (c.f. Figure 18).

Polysynaptic reflexes provide an organism with the possibility to react on a stimulus in a fast, unconscious way, involving the integration of information by sensory organs and structures. This pattern is often found in protective behavior. Motor behavior as an outcome of a polysynaptic reflex arc is modified in various ways (Perl, 1962). These reflexes occur in diverse ways with equally various complexities of involved neural circuitries. Interneurons mediate this information between neural circuitries. Microscopic findings indicate that about 30 interneurons are connected to one 
Introduction

motoneuron. Interneurons can be grouped into several subtypes: (1) local interneurons have a limited spatial effect, (2) propriospinal interneurons appear with a long-range effect, (3) commissural interneurons ensure bilateral information coordination and (4) interneurons that traverse over large ascending axons to higher parts of the CNS (Kandel et al., 2012).

Kandel et al. (Kandel et al., 2012) describe, that polysynaptic reflex circuits occur in various shapes. For instance, an unconscious protective reflex is the blink reflex, where differently involved neural structures transmit information that leads finally to contraction of the $M$. orbicularis oculi and subsequent to shut of the eye lid; since the eyelid does not possess any supporting muscles and muscle contraction takes place via several neural circuitries, this reflex is also mentioned as polysynaptic reflex (Kandel et al., 2012). An example for a conditioned reflex is found in Pawlow's dog experiments where dogs were conditioned to ringing of a bell according to food granting; also higher brain centers are involved in the learning process which affect motor behavior according to the learned stimulus. Other types of reflexes involve more muscle groups to achieve an adequate reaction to a stimulus. This is found in primitive reflexes, for instance. These reflexes describe a reproductive reaction pattern of, for example, protective motor behavior. This type is found in rooting, sucking, palmar, plantar, swimming and other reflexes (Kandel et al., 2012). 
Introduction

\subsubsection{The spinal circuitry between lower limb extensor and flexor muscles}

In order to perform different types of movement, fine muscle interplay as well as co-contractions, the lower limbs' extensor and flexor muscles must be able to achieve neuromuscular adaptations according to the needed function for the entire system. In the case of locomotion, a continuous interplay of extensor and flexor muscle by excitation and inhibition is of fundamental importance to allow movements of muscles, joints and the execution of the desired movement. Motoneurons in vertebrates are exclusively excitatory and cholinergic. Therefore, the organism must accomplish active muscle relaxation by information flow via interneurons, which serves as a mechanism to actively control muscle contraction or relaxation (Kandel et al., 2012).

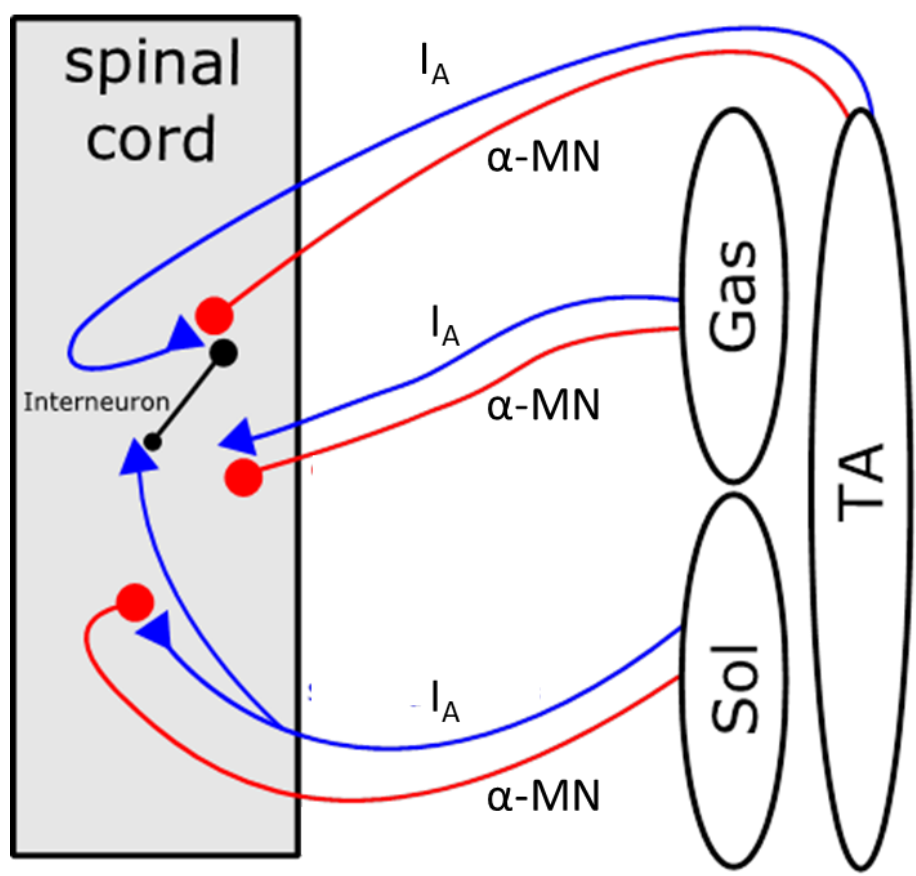

Figure 17: Schematic overview of inter-connectivity of lower limb afferent and efferent fibers to asure smooth muscle interplay. Reciprocal inhibition of TA by TS shown exemplary. Afferents colored in blue, efferents colored in red.

Figure 17 depicts the neural circuitry of the lower limb muscles we examined in our experiments (Yavuz et al., 2017, 2018). The lower limbs' calf muscles are shown as SOL and GM, which build up the TS muscle group together with the M. plantaris. These muscles contribute to plantar flexion and supination of foot and ankle. Dorsal extension as well as knee stabilization is accomplished by TA located near the shin. The big muscle group of TS and TA are interconnected to provide online control and crosstalk between both.

During voluntary movement, $\alpha$-motoneurons are activated reciprocally via la-afferents (Knikou, 2008). It was found that least one neuron serves as interneuron in animals (Eccles et al., 1956) and in human (Crone et al., 1987; Katz et al., 1991) to connect the partial agonistic muscles. Later on, it was shown that excitatory and inhibitory interneurons provide the possibility to achieve smooth gait 
Introduction

(Katz et al., 1988; Capaday et al., 1990). Investigations focused on the reciprocal inhibition itself but not on the mutual distribution of inhibitory reciprocal input. With our approach, we analyzed the excitability of the motoneuron pool and the distribution of synaptic inputs and intrinsic properties of $\alpha$-motoneurons. Extensor and flexor muscles of the lower limb differ in a vast way from each other, i.e. number of muscle subgroups, anatomy, physiological character, synaptic input distributions etc. We can assume an asymmetric reciprocal inhibition between extensor and flexor muscles, as former studies indicated, using sEMG and single motor unit analysis (Crone et al., 1987; Nielsen et al., 1992). But these studies do not answer the question, if the asymmetric reciprocal inhibition arises from different mutual distribution of reciprocal inhibitory input. To examine this gap, we designed our experiments with measurement of standardized input on timings of discharge of individual motor units and by sampling a large count of activated motor units (Nielsen et al., 1992; Yavuz et al., 2015). Our study 1 investigated the interplay between extensor and flexor muscles of the lower limb and we found a non-symmetrical distribution of inhibitory reciprocal reflex responses (Yavuz et al., 2017, 2018).

It should be mentioned as well, that long-term motor skill training leads to particular refinements and adaptations of neural structures; this is thought to arise from plasticity of involved afferent and efferent fibers (Nielsen et al., 1993 b; Adkins et al., 2006; Mazzocchio et al., 2006; Wolpaw, 2007). By learning a new motor task afferent and efferent fiber's drive is refined to accomplish smooth movement trajectory (Pierrot-Deseilligny et al., 2012).

\subsubsection{Motor modules}

The term of motor modules (i.e. synergies) should be briefly introduced to provide the neuromuscular context of motor behavior. Muscle synergies are thought to represent motor modules, that are recruited by the nervous system to perform motor-subtasks, which are necessary for movement (Safavynia et al., 2011).

Walking and running, which build up the repertoire of two types of human locomotion, are controlled by shared, pattern-generating networks (Cappellini et al., 2006). The measurement of EMG activity of different muscles, using multi-electrode arrays in locomotion, enables the investigator to examine the activity of synergistic muscles that lead to the performance of a specific movement. As described for gait, several motor modules are described (Oliveira et al., 2012). Since human locomotion consist of different phases of walking, it went to great interest to understand the underlying control mechanisms of those motor modules (Ivanenko et al., 2005; Cappellini et al., 2006; Lacquaniti et al., 2012; Oliveira et al., 2013). 
Introduction

Since this branch of physiology and kinesiology represents an entire field of research, it should be mentioned and briefly introduced since the importance for neuromuscular control is very evident. It should also be taken into account for observations in terms of neuromuscular adaptations and in regard of long-term motor skill training. In our experimental setup we analyzed rather the behavior of motor modules but focused on the underlying physiological behavior of single motor units.

\subsection{Spinal plasticity affects neuromuscular properties}

Due to long-term motor skill training, the spinal cord shows neuroplasticity in a sense of change in neural drive, which is mainly associated with synaptogenesis (Adkins et al., 2006). It is known that reflex amplitudes and therefore muscle interplay in skilled long-term trained ballet dancers is significantly different from control (Nielsen et al., 1993 b). Functional and neural changes according to long-term motor skill training like martial arts (Sørensen et al., 1996; Zehr et al., 1997; Sforza et al., 2002; Gatts, 2008) were investigated, as well as body arts like Yoga (Williams, 2009; Ni et al., 2014), as well as passive or active stretching exercises (Avela et al., 1999 a; Guissard et al., 2006; Robertson et al., 2012; Dupeyron et al., 2013; Balle et al., 2015; Behm et al., 2015; Zotz et al., 2016). Results are indicating that neuromuscular adaptations take place at different stages of development in various neuromuscular structures as muscle properties, afferent and efferent excitability, neural drive, interneuron pathways etc.

We accept these adaptations to take place in a group of regular long-term trained Ninjutsu participants since literature indicates differences in motor behavior due to training experience (Nielsen et al., 1993 b; Augé et al., 2000; Schneider et al., 2002; Adkins et al., 2006; Ogawa et al., 2009). These changes must underlie different neuromuscular control mechanisms, since the outcome of these mechanisms is defining how motor actions are (functionally) performed. This makes a group of skilled long-term trained martial artists, as well as a group of Yoga skilled practitioners, to an ideal object of study to analyze underlying neuromechanical differences in relation to the proprioceptive feedback system involving $I_{A}$ afferent fibers.

\subsubsection{Investigation of motoneuron excitability and spinal plasticity}

By usage of monopolar recorded reflexes as a probe, we are able to get insight into motoneuron excitability and spinal plasticity (Knikou, 2008). Recording of electrically evoked H-reflexes allows the investigator to record and investigate the signal by filtering electrical noise. The experiment's 
Introduction

outcome is the amount of voltage which spreads over the muscle belly (i.e. EMG recording). This is used to make a statement about the global firing event, resulting in an electrical volley evoked by all activated motoneurons (Hoffmann, 1910; Knikou, 2008). This technique allows investigation of neural plasticity on spinal level and is a robust, relying and accepted way of investigation (Hoffmann, 1910; Capaday et al., 1990; Nielsen et al., 1993 b; Avela et al., 1999 a; Wolpaw, 2007; Knikou, 2008).

In our experiments we investigated with a standardized amount of current, applied to TN in the popliteal fossa, and with a differential measurement method, the amount of voltage between two electrodes (out of 64 available electrodes on the array). The resulting direct motor response (Mwave), respectively the elicited reflex in the homonymous muscle (H-reflex), is depicted in Figure 18.

\subsubsection{M-wave and H-reflex}
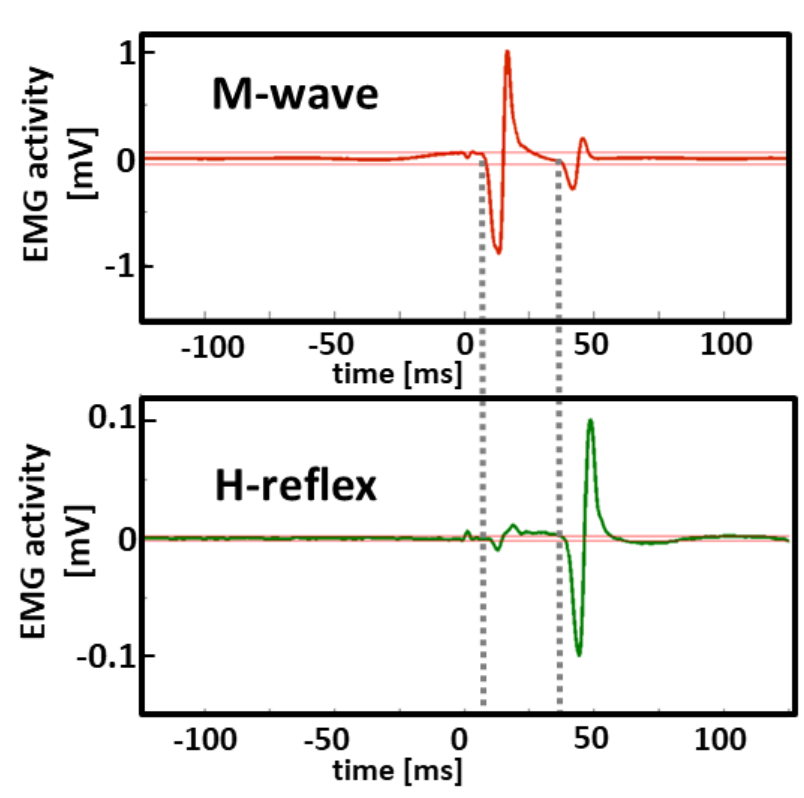

Figure 18: Explanation of excitation of the mixed nerve TN. Shown is the time curve of EMG 100 ms before and after the stimulation. Upper trace (red) depicts direct motor response (M-wave), lower trace (green) depicts reflex in the homonymous muscle (H-reflex); excitatory stimulus applied at time mark $0 \mathrm{~ms}$.

High electrical stimuli cause direct motor response (M-wave) by direct excitation of motoneurons and afferents, whereas low electrical stimuli only stimulate afferent fibers (H-reflex) (Knikou, 2008). Direct motor response occurs immediately after the excitation; $\mathrm{H}$-reflex staggered about 50 ms later (c.f. Figure 18 and Figure 19). Direct motor response is elicited if the electrically applied stimulus is strong enough to excite $\alpha$-motoneurons directly (c.f. Figure $\mathbf{2 0}$ ). If so, $\mathrm{I}_{\mathrm{A}}$-afferent fibers are excited as well. But since the electrical signal propagates orthodromic (elicited by $I_{A}$ afferent fibers) and antidromic over the motoneuron, it collides with the AP generated at the spinal cord on the homonymous motoneuron leading to an elimination of the physiologically generated AP. Only the part of the signal propagating from the excitation area to the muscle (orthodromic) causes muscle 
Introduction

contraction. This measureable direct motor response is called M-wave and appears between 5 to $10 \mathrm{~ms}$ after the excitation in the popliteal fossa (Schmidt et al., 2007). The time-interval between the direct motor response and the onset of force development is called electromechanical delay (EMD) (Yavuz et al., 2010).

Besides this, also the voluntary drive ( $V$-wave) from cortex should be mentioned. The voluntary drive appears during maximal contractions with supramaximal stimuli and is mentioned as a variant of $\mathrm{H}$ reflex (Fimland et al., 2009); V-wave is thought is represent the magnitude of efferent neural drive, which can be altered by the execution of training (Fimland et al., 2009).

By lowering the cutaneous applied excitation intensity, motoneurons with smaller diameter are excited first (Henneman et al., 1965). On the contrary, afferent type I and type II fibers are activated from largest to smallest, due to their lower threshold (Kandel et al., 2012).

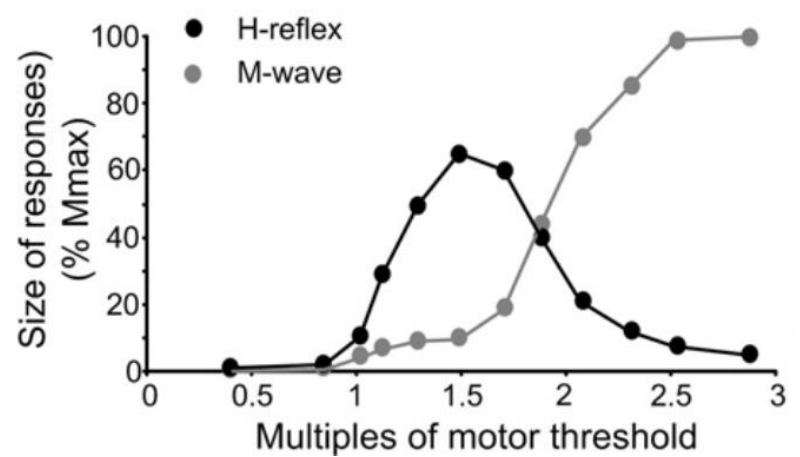

Figure 19: Depiction of reciprocal action in the increase in stimulation intensity between the size of motor responses of $\mathrm{H}$ reflex (black) and $\mathrm{M}$-wave (grey) as multiples of motor threshold, caused by excitation of the peripheral mixed nerve TN (by Knikou, 2008, modified).

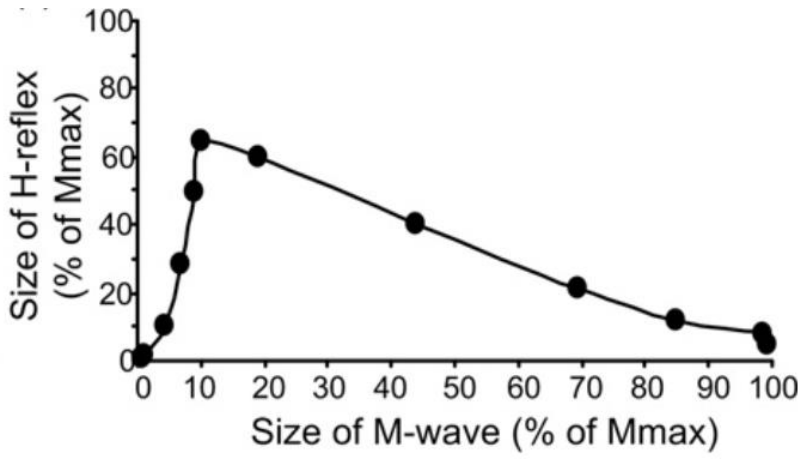

Figure 20: Depiction of the relation between size of $\mathrm{H}$-reflex and size of M-wave (by Knikou, 2002, modified).

\subsection{Context of the study}

\subsubsection{Motor behavior and environment}

Motor behavior describes the behavior of an organism in respect of its motor control and motor system (Kandel et al., 2012). This is present in very different areas of everyday life. It is crucial for the organism to live and interact with the environment, develop and spread.

Every organism enters the world at birth with a repertoire of modifiable motor behavior. This repertoire is for example present in the animal kingdom when we observe quadrupeds getting on 
Introduction

their feet as their very first task to accomplish. But also humans have impressive abilities at the stage of a newborn. Several reflexes are present, for example: palmar grasp reflex, sucking reflex, plantar reflex, swimming reflex etc. (Schmidt et al., 2007; Kandel et al., 2012). Most of these reflexes disappear during the first months after birth, getting replaced by motor behavior from higher levels since synaptoplasticity is highest in the first days after birth. From the stage of birth continuing over the lifespan to elder organisms, neural plasticity is reduced, but it is still present (Lohmann et al., 2014). This makes learning new tasks and implementing motor programs more challenging, but it is still possible.

Motor action is the most important factor in the development of animals and humans. Without motor actions we would not be able to live in and interact with our environment. Motor behavior is the main driving force of art and therefore for emergence of human culture in written word, painting, body arts etc. All expression of human mood and transmission of social topics and history are accomplished by motor behavior. This genesis would not have been possible without the development, altering and execution of motor tasks. If we take a look not in past but in present and future, the planning of movements and learning new skills comes up to us. Even when dreaming the motor cortex is highly active (De Carli et al., 2016). This makes sense, if we take into account that all dreams are in action and not a still life.

\subsubsection{The effect of training on spinal plasticity}

Since the interest of this study is the plasticity of the spinal cord, we investigated a group of skill trained martial artists and compared them with a group of moderately active people. The training of the martial arts group consists of three different training areas, with regard to the allocation Adkins et al. made (Adkins et al., 2006). Training usually involves all three mentioned areas of skill, power and endurance training, no matter which task is preferably performed (c.f. Error! Reference source ot found.). But when it comes to groups which specifically train for one parameter, the two other are usually shortened in training, but they are still evident. However, Adkins et al. (Adkins et al., 2006) defined the neural results for those three types of training on the spinal cord level for skill, strength and endurance training. These training types are explained in the following.

\subsubsection{Skill training}

By operant conditioning of the spinal stretch reflex (H-reflex), Wolpaw and colleagues made the finding that spinal plasticity persists in monkeys (Carp et al., 1995), rats (Chen et al., 2001) and 
Introduction

human (Segal et al., 2000) and even after transection in primates (Wolpaw et al., 1989; Wolpaw, 2007). H-reflex changes are a common parameter to investigate spinal plasticity with regard to longterm training (Rochcongar et al., 1979; Nielsen et al., 1993 b; Augé et al., 2000; Mazzocchio et al., 2006; Ogawa et al., 2009) as well as to 10 days of training exercise in walking backward (Ung et al., 2005). A conditioned decrease in $\mathrm{H}$-reflex size occurs due to increasing motoneuron firing threshold, whereas a reduced inhibition of a motoneuron results in an increase (Adkins et al., 2006).

Since skill training affects spinal reflexes, which occur entirely in spinal cord pathways, the spinal cord itself is the ideal region to perform research on neural plasticity with regard to skill training (Thompson et al., 2014). By taking these findings into account, re-organization and synaptoplasticity takes place in the spinal cord as a result of skill training.

\subsubsection{Strength training}

While comparing power-trained athletes with endurance trained athletes (Maffiuletti et al., 2001), it was found, that a reduced H-reflex excitability is present in the power-trained group, where on the other hand the maximum voluntary contraction level is increased in this group.

Power training, i.e. strength training, is thought to increase overall participating motor units and the output of force; also it seems to compensate the lower efficacy of the reflex transmission between $I_{A}$ spindle afferent input and $\alpha$-motoneuron of SOL (Maffiuletti et al., 2001).

The V-wave (c.f. chapter 2.6.2) represents the corticospinal drive. Experiments show evidence that the corticospinal drive increases within 3 weeks of strength training (Vila-Cha et al., 2012) and after 9 - 21 weeks (Sale et al., 1983). By comparing skill with strength training Remple et al. found evidence, that skill training but not strength training induces changes in spinal plasticity (Remple et al., 2001). Taking this into account, strength training seems to reinforce existing synapses and leads to increased excitability of motoneurons.

\subsubsection{Endurance training}

Endurance training leads to an increase in H-reflex size as well as Achilles tendon and patellar tendon reflex activity compared to control (Koceja et al., 2004). Findings by Walton et al. point out that endurance-trained athletes perform with a higher reduction in H-reflex amplitude compared to sedentary while executing a fatiguing protocol (Walton et al., 2002). 
Introduction

\subsubsection{Ninjutsu}

We have chosen a group of regularly training martial artists of Ninjutsu. Ninjutsu is an eastern martial art with its origin in province of Iga, Japan. The system of Bujinkan Budo Taijutsu, also called Bujinkan Ninjutsu, is a martial art system which combines six samurai schools with three ninja schools. Emergences of schools reach back in time until around 1000 AD (Hatsumi et al., 2008).

We focused on the outcome of nowadays training of Ninjutsu in terms of physical and neural aspects. In general, training involves physical training for building up stamina and fatigue, as well as training how to fall and break falls and the technique training itself.

The following parts of training are mainly performed in solo training. Physical training leads to more skill, power and endurance. Learning how to fall (break falls, leaping, dodging) provides the executioner possibility to sense how the movement is bodily performed in an accurate and proper way. Technique training is usually performed in postures or stances. These stances are performed with a lowered center of mass (COM) by standing with knees slightly bend. All techniques require a lowered CoM compared to normal walking or standing. Besides this, appropriate kicking and punching is trained to improve control itself and control of the expected result (Arus, 2013). Training with a partner is usually performed to train techniques of self-defense (c.f. chapter 2.7.6.2).

\subsubsection{Explanation of the concept}

The concept of Ninjutsu leads to a refinement of movements itself due to training, achieved by conscious or unconscious improvement of sensing the body in terms of position, force and motion in space - similar to the Feldenkrais Method (Feldenkrais, 1972).

\subsubsection{Training in martial arts}

A common training session of two hours is designed as follows: 30 min warm-up, 30 min break-falls and 60 min technique training. In all parts of the session standing in lowered body positions (Figure 21) - and therefore frequent activation of the lower limb muscles - is demanded. Subjects examined in this study are training at least in two or three of these sessions each week, in at least the last three years until now, and are holding a black belt.

The warm-up consists of typical cardio training (walking, running) combined with moderate power and strength training (squats, sit-ups, push-ups etc.). This unit is followed by learning of break-falls. For instance, subjects are standing in adequate lowered body positions while moving into a roll (for 
Introduction

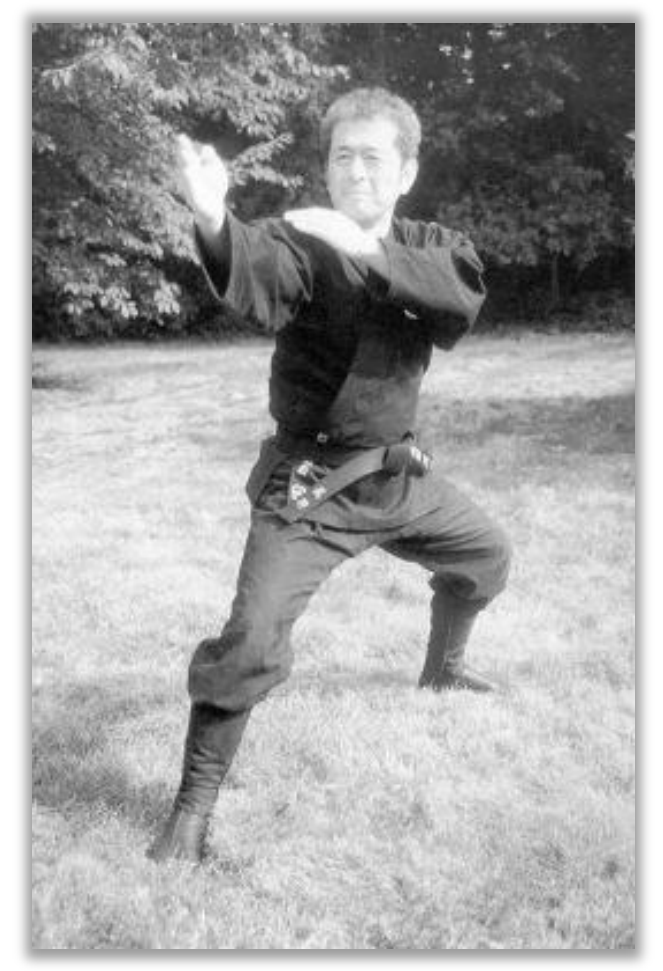

Figure 21: Photograph of Ninjutsu grandmaster and founder PhD Masaaki Hatsumi, performing the a stance of the system Bujinkan Budo Taijutsu (from https://shugyoudojo.files.wordpress.com/2011/02/soke-kamae.jpg accessed $1^{\text {st }}$ June 2018).

dodging or evading), standing up and adopting a stance with lowered CoM again, therefore accessing muscles of knee and ankle stabilization (i.e. GM and SOL). In the following unit, the technique training, appropriate moving, as well as techniques of self-defense is trained (partner training). Since techniques of self-defense are only executable with success, if a lowered CoM compared to the opponent's CoM is taken up as a stance, lower limb muscle are heavily accessed throughout the entire training session, especially while technique training (Hatsumi et al., 2008).

\subsubsection{Training in stretching and Yoga}

It is commonly known and reinforced by scientific and medicinal research, that long-term Yoga training leads to an improvement in well-being and alleviation of lower back pain (Williams, 2009). Practitioners of Yoga usually train in a way where lower limb, rear and lower back muscles are strengthened as well as stretched. A typical training session of one hour consists of a light warm-up by adopting postures which access a few connected structures, followed by more complex positions accessing more body structures (c.f. Figure 22).

In this type of training, skill is practiced as well as in in the group of martial artists. Training techniques in different Yoga styles vary from each other, but with motor skill training neuromuscular 
Introduction

structures and neural drive are altered in general (Nielsen et al., 1993 b; Carp et al., 1995; Adkins et al., 2006).

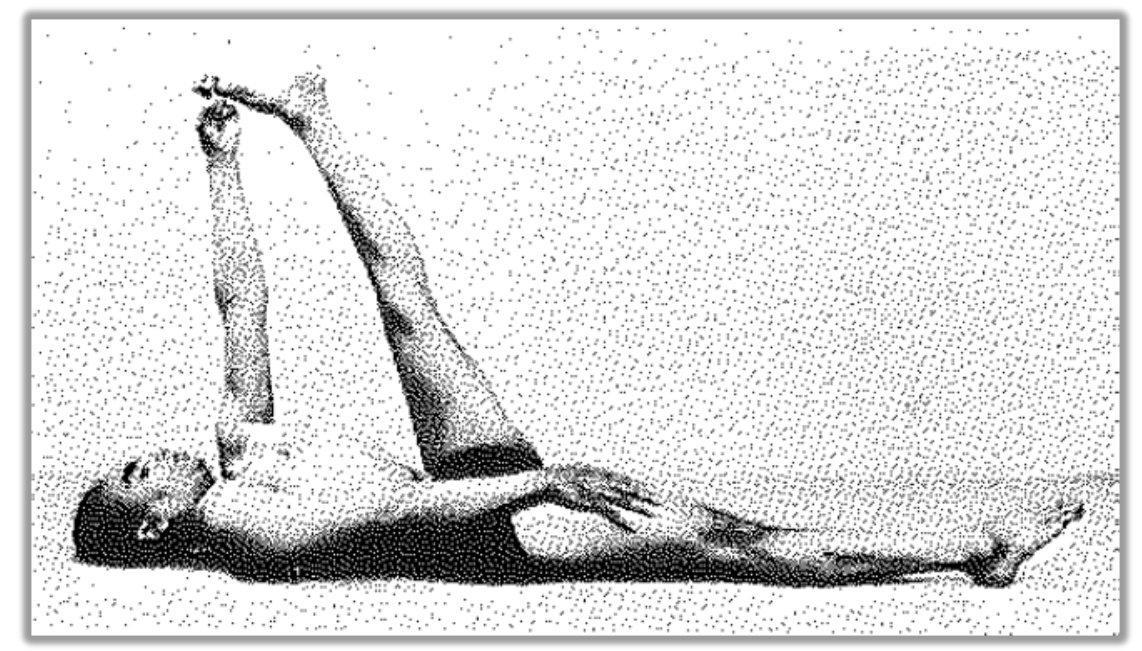

Figure 22: Photograph of B. K. S. Iyengar, founder of the style lyengar Yoga, performing a basic stretch technique (called Supta-Pādāngushthāsana) accessing are mainly muscles of the posterior chain (from http://www.liveyoga.nl/wpcontent/uploads/

\subsection{Materials and Methods}

\subsubsection{Experimental setup}

In the three presented studies the following setup and equipment was used.

\subsubsection{Subject position}

The Biodex System 3 Pro $^{\circledR}$ (Biodex Medical Systems Inc., NY, USA) can be applied for broad types of investigations (isometric and isokinetic) at different parts of the body (like knee, ankle, hip, shoulder, elbow, forearm and wrist). We used the system to determine isometric contraction force of the participant, while providing optical feedback of torque output. This gives us possibility to standardize the voluntary contraction level of each participant in respect of their individual maximum voluntary contraction force (Yavuz et al., 2014, 2015, 2018).

Seated on a chair, the participant's ankle was provided a reference electrode (WS2, OTElettronica, Torino, Italy) and the joint was fixed in the anatomical resting position. Knee angle was $120^{\circ}$ for SOL, GM and TA activation, respectively $170^{\circ}$ for GM activation. Torque development was recorded with a dynamometer. Stimulation electrodes were placed with metal pin anode and cathode on the popliteal fossa (for stimulating TN) and on the head of the fibula (for stimulating CPN). Conductive gel (AC CREAM250V ${ }^{\circledR}$, spes medica, Genova, Italy) was used to improve contact between metal pins and skin. 


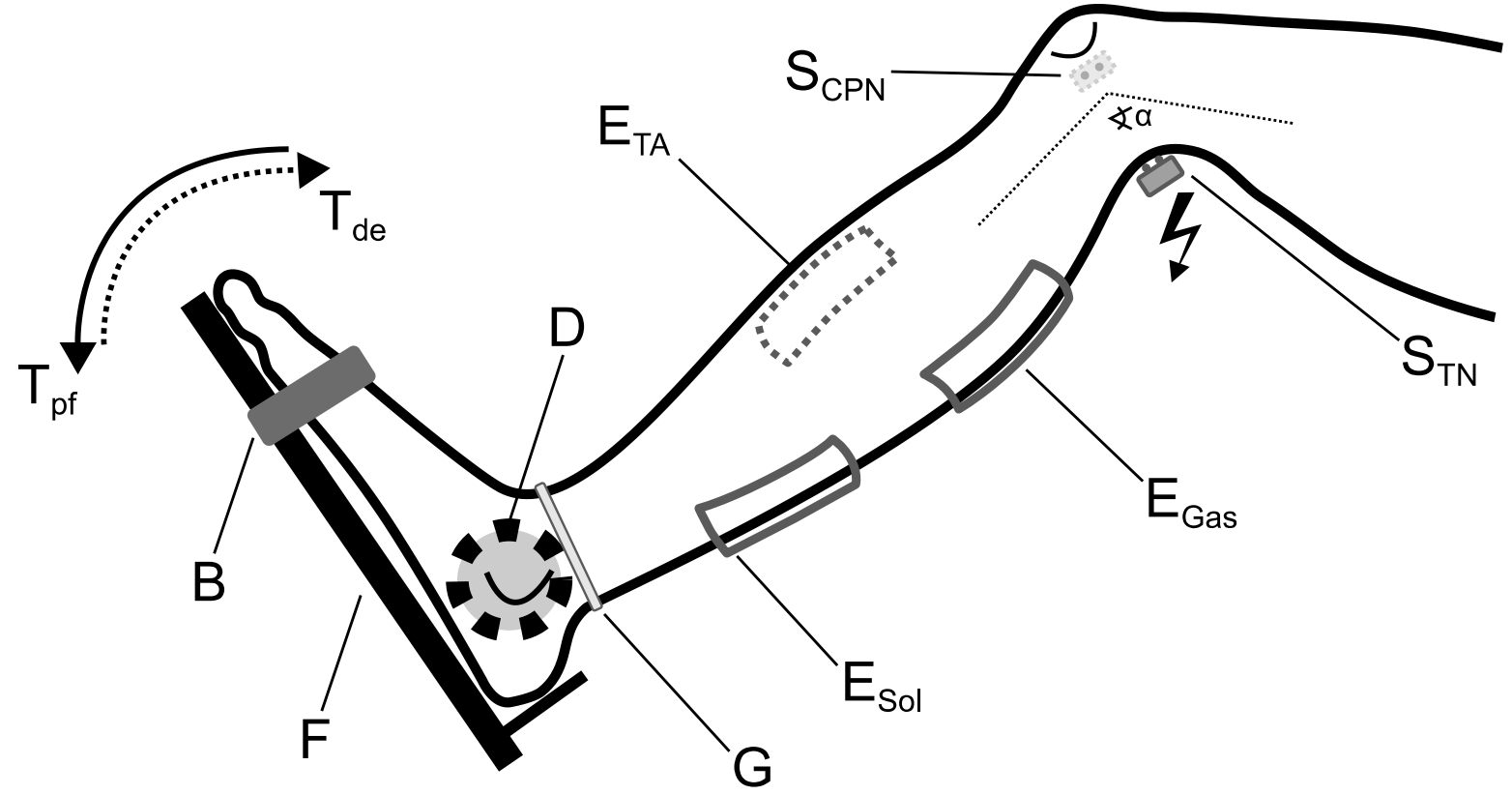

Figure 23: Schematic overview of the subjects' right leg while seated in Biodex System 3 Pro ${ }^{\circledR}$ during $\mathrm{H}$-reflex measurement; explanation and abbreviations in floating-text.

Figure 23 depicts a performed measurement at the Biodex System $3 \mathrm{Pro}^{\circledR}$ while the subject performs sustained contraction and H-reflex is measured. The participant's foot will rest on the footplate (F) with a fixing belt (B) to keep the ankle in its resting position. The footplate is attached to a dynamometer (D) to measure torque development for plantar flexion $\left(T_{\mathrm{pf}}\right)$ and dorsal extension $\left(T_{\text {de }}\right)$ which is proportional to the developed force levels. Three HDsEMG electrodes are mounted on the lower limb muscles $M$. soleus, $M$. gastrocnemius and $M$. tibialis anterior $\left(\mathrm{E}_{\mathrm{Sol}_{0}}, \mathrm{E}_{\mathrm{Gas}}, \mathrm{E}_{\mathrm{TA}}\right)$. Knee angle $(\alpha)$ is $120^{\circ}$, respectively $170^{\circ}$, stimulation electrode $\left(S_{T N}\right)$ is mounted in the popliteal fossa next to the tibial nerve (c.f. Figure 5); respectively on common peroneal nerve $\left(S_{C P N}\right)$.

\subsubsection{Stimulation}

The Digitimer DS5 ${ }^{\circledR}$ (Digitimer Ltd., Hertfordshire, UK) is an isolated bipolar constant current stimulator which is used to investigate clinical nerve excitability. This system allows us to generate repetitive square pulses with duration of $0.003 \mathrm{~ms}$. Controlled by a MatLab-script, power output is defined to achieve proper stimulation intensities in the range of $12-16 \mathrm{~mA}$. We use this system to apply stimuli to TN located in the popliteal fossa (c.f. Figure 5, Figure 23), and CPN, located at the outer side of the knee (c.f. Figure 23). TN, either for reciprocal inhibition of TS muscle group or for excitation of the homonymous muscle, and CPN, for reciprocal inhibition of TA, were stimulated in different sessions. A minimum of 150 pulses (square-wave pulse, $0.3-0.5 \mathrm{~ms}$ pulse duration, $1-2 \mathrm{~s}$ random inter pulse interval) were delivered to the mixed nerve of TN, respectively CPN. Sessions were randomized. Anatomically, these stimulation points are easy to locate. CPN was stimulated by 
Introduction

placing the stimulation electrode's anode and cathode on the anterior and posterior part of fibula's head, whereas for stimulation of TN the stimulation electrode was placed in the popliteal fossa. $\mathrm{H}$ reflex/M-wave-recording (c.f. Figure 18) of the desired muscles provided us information to adjust and standardize stimulation intensities to $\mathrm{H}$-reflex size of $10 \%$ of the maximum direct motor response size (Yavuz et al., 2015).

\subsubsection{Electrode preparation and EMG recording}

Before placing the EMG surface electrodes, the regions of the expected mounting position were prepared by shaving hairs, cleaning the skin with abrasive gel (EVERI160SPE ${ }^{\circledR}$, spes medica, Genova, Italy) and alcohol pads (B. Braun, Melsungen, Germany). After alcohol evaporated, the electrodes, coated with conductive gel (NEURGEL250V, spes medica, Genova, Italy), were mounted.

Reducing the multichannel-recording, by exclusion of channels, into a bipolar sEMG measurement was only performed when decomposition into single MUs was not necessary; Laplace operator was used for this purpose.

Shape and amplitude of the $\mathrm{M}$-wave/H-reflex by excitation via $\mathrm{I}_{\mathrm{A}}$-afferents on the homonymous muscle can be investigated by using single sEMG electrodes as control to tune stimulation intensities accordingly (Adkins et al., 2006). For this purpose we used either single channel Ambu ${ }^{\circledR}$ Neuroline 720 (Ambu, Bad Nauheim, Germany) surface electrodes (c.f. Figure 24), if no further calculation of this muscle into single MUs is needed, or displayed the output of a single electrode of high density multichannel surface Adhesive Matrix ELSCH064NM2 (Bio Elettronica, Torino, Italy) density surface electrodes (c.f. Figure 25).

For the recordings and further calculation of single MUs we used high density multichannel surface electrodes. The inter-electrode distance between each electrode is $0.8 \mathrm{~mm}$; layout of pins is $5 \times 13$ electrode grid. Electrodes were placed as shown in Figure $\mathbf{2 3}$ on the muscle belly of SOL, GM and TA. Measurements were performed in differential mode. 


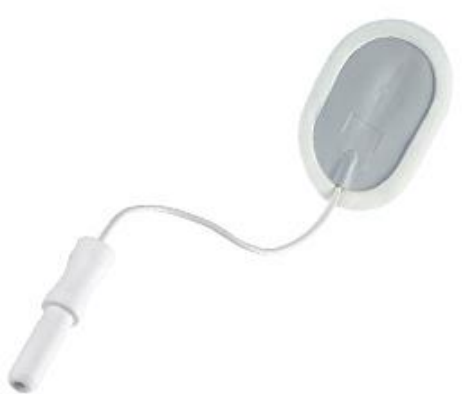

Figure 24: Single channel sEMG electrode of type $\mathrm{Ambu}^{\circledR}$ Neuroline 720 (from http://www.ambu.de/Files/Billeder/ Product\%20Images/720Large.jpg accessed $1^{\text {st }}$ June 2018).

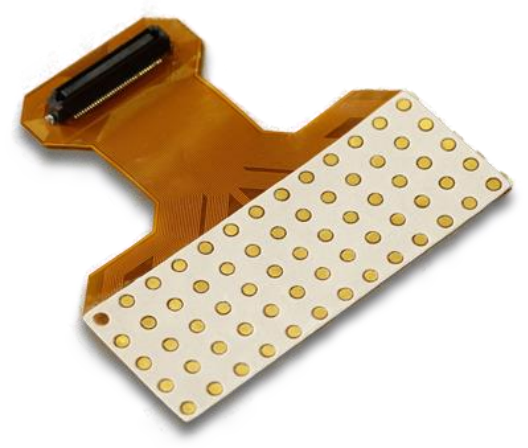

Figure 25: Multichannel sEMG electrode of type Bio Elettronica ${ }^{\circledR}$ ELSCH064NM2

(from http://www.otbioelettronica.it/images/prodotti/ Elettrodi/ELSCH064NM2.png accessed $1^{\text {st }}$ June 2018)

\subsubsection{Data acquisition}

We used for bipolar measurements preamplifier AD2x8JD (OTElettronica, Torino, Italy), for multichannel HDsEMG electrodes preamplifier AD1x64SD5 (OTElettronica, Torino, Italy). The signal was amplified using a multichannel EMG data acquisition system (EMG-USB2 amplifier, OTElettronica, Torino, Italy). Amplified signals were band-pass filtered at $20-500 \mathrm{~Hz}$ and recorded with a Microsoft Windows ${ }^{\circledast}$ PC with OT Elettronica ${ }^{\circledR}$ recording software. Sampling rate for trigger and force signal with 12 bps was $10240 \mathrm{~Hz}$, as well as EMG data. The signal was provided for the participant as optical feedback of torque, which represents the exerted amount of force. This we did, to perform the sustained contraction at $10 \%$ or respectively $20 \%$ of their MVC. For this purpose we used a custom made MatLab ${ }^{\circledR}$ (The MathWorks, Inc.) script as well as acquiring M-wave/H-reflextuning-curve to elicit henceforth $\mathrm{H}$-reflexes with a size of $10 \%$ of the maximum direct motor response. By tuning the stimulation intensities in respect to the excitation of la-afferents only, trials with clear H-reflexes and no appearance of M-wave were analyzed. Trials contaminated with $\mathrm{M}$ waves were discarded.

For further data examination the recorded data at $10240 \mathrm{~Hz}$ were downsampled to $2048 \mathrm{~Hz}$ and divided into intervals of $60 \mathrm{~s}$ with $10 \mathrm{~s}$ overlap and decomposed separately (c.f. chapter 2.9.1). Spiketrains were merged if the cross-correlation coefficient was greater than $90 \%$ (Yavuz et al., 2015). We calculated PSF-CUSUM $400 \mathrm{~ms}$ around the stimulus $( \pm 200 \mathrm{~ms}$ ). For the examination of reciprocal inhibition (study 1), the PSTH was used to confirm the onset of inhibition, whereas the strength of inhibition was analyzed with PSF-CUSUM. In study 2 the PSTH was used to determine the onset of the excitation of the homonymous muscle. Mean discharge rates (study 1) were calculated individually in the pre-stimulus time interval $(500 \mathrm{~ms})$; maximum PSF-CUSUM deviation in peristimulus time frame was used to determine the significance of inhibition strength. We only accepted 
Introduction

troughs that exceeded significance threshold as genuine responses (Yavuz et al., 2018). In study 3 we used a goniometer to define the differences in stretching angle. EMG data were either recorded using HDsEMG electrodes or bipolar SEMG electrodes. HDsEMG data were digitally converted for further analysis, using Laplace operator, into bipolar electrodes. For the analysis of MUDR, pooled data of all reliable MUs between groups were used. We accepted only those MUs as reliable, which fire in physiological ranges (Kandel et al., 2012). EMD was calculated from HDsEMG recordings, using the time-interval between the application of a supramaximal stimulus and the exertion of force.

\subsubsection{Force recording and feedback system}

The participant was asked to perform voluntary sustained plantar flexion with a certain amount (10 or $20 \%$ ) of their MVC. Force was recorded with a dynamometer allowing measurement of force as a function of torque development, which is part is the Biodex system. Force feedback was provided by a self-conceptualized MatLab-script via a monitor. The settings were chosen in a way to allow visualization of the double amount of force exerted by the subject to ensure that ramp parts are displayed and executed properly.

\subsection{Data analysis}

\subsubsection{Motor unit decomposition}

For signal processing, a test $\mathrm{H}$-reflex in the homonymous muscle was recorded (c.f. Figure 7) during sustained contraction. The HDsEMG signal of the homonymous muscle was recorded with corresponding trigger pulses (c.f. Figure 26). 


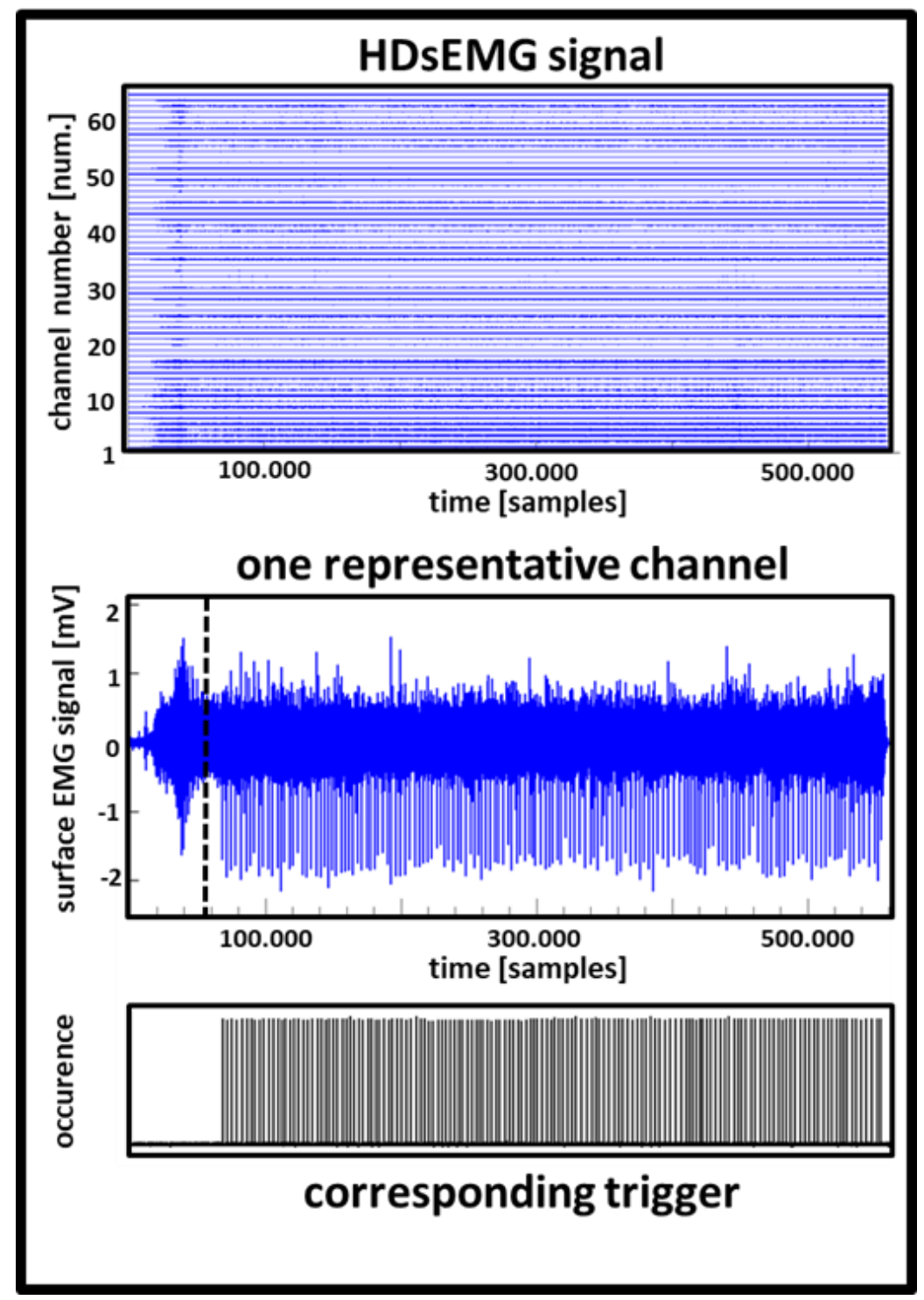

Figure 26: Illustration of HDSEMG recording from M. soleus during experiment; dashed vertical line represents end of ramppart and beginning of application of trigger pulses.

Figure 26 depicts the recorded excitatory signal of SOL of a HDsEMG electrode in differential mode with 63 channels displayed (top). Since we used 64-channel electrodes and recorded in differential mode to reduce background noise, all available 63 channels are shown. One channel is examplary plottet (middle) to visualize EMG activity just before the experiment starts, ramp-parts and effect of incoming trigger pulses (bottom), triggered by the excitation electrode in the popliteal fossa.

Recorded data were checked manually with a custom made MatLab (The MathWorks, Inc.) script for signal quality and broken channels in HDsEMG recordings. Broken channels were excluded from further analysis. Data for HDsEMG electrodes were down-sampled from 10240 to $2048 \mathrm{~Hz}$ and Butterworth-filtered between 20 and $500 \mathrm{~Hz}$. HDsEMG signals were decomposed, using a gradient- 
Introduction

based convolution kernel technique (Holobar et al., 2007 a) providing fully automatic decomposition, to extract the activity of single motor units from the HDsEMG recording.

For this purpose we used DEMUSEtool ${ }^{\circledR}$ (Holobar et al., $2007 \mathrm{~b}$; a). The tool works with a blind source separation technique (Holobar et al., 2007 a; b; Negro et al., 2016) and provides fully automatic decomposition. Its reliability is proven in several studies (Holobar et al., 2007 b, 2012; Farina et al., 2010; Negro et al., 2016) and it allows us, depending on the specific individual discharge rate of each $\mathrm{MU}$, to separate individual motor units from global EMG recording; this procedure is called decomposition. In the decomposition of HDsEMG signals, the convolution kernel compensation method was used to deal with the mixtures of motor unit innervation pulse trains in low-quality and noisy EMG-signals (Holobar et al., 2007 b). Signals were manually divided into epochs of $60 \mathrm{~s}$ with an overlap interval of $10 \mathrm{~s}$. After decomposing epochs into individual spike trains, the common motor units between epochs were estimated computing cross-correlation. The matches with correlation coefficient $>90 \%$ were assumed as the same motor unit and merged through epochs (Yavuz et al., 2015).

\subsubsection{PSTH, PSF and CUSUM calculation}

In this chapter we highlight peristimulus time histogram (PSTH), peristimulus frequencygram (PSF) and cumulative sum (CUSUM) calculation (c.f. Figure 27). PSTH is a reliable tool to analyze the probability of occurrence of MU discharges in neurosciences (Katz et al., 1988; Capaday et al., 1990; Nielsen et al., 1993 a; Rogasch et al., 2012). It is used to analyze extracellularly recorded action potentials; it appears as a correlogram of stimulus and discharge pulse trains, built up by a number of trials (Ellaway, 1978). The underlying bin-width depends on how detailed information will be accessed.

It turned out that the cumulative sum technique applied to PSTH (PSTH-CUSUM, not shown in Figure 27), yields information with less contamination, compared to PSTH (Awiszus et al., 1991). Sequent research revealed even more robust and reliable information of $\mathrm{MU}$-behavior with information of the frequency a MN discharges with (Türker et al., 1994; Yavuz et al., 2015) (compared to the all-ornone-event depicted in PSTH), again built up by a number of trials, which builds up PSF.

PSF is of great importance to analyze the instantaneous discharge rates of motor units, since ratecoding of the motoneuron defines the motor unit's firing rate (Kandel et al., 2012), ranging from single twitches to tetanic contraction of muscle fibers. Applying the technique of CUSUM to PSF provides information of MU-discharge, depending on the discharge-frequency. For building up PSF- 
Introduction

CUSUM, a period of stable activity is required to calculate an average baseline; the differences between averaged frequency and the frequency of each bin (derived from differences between sum of changes in frequency and baseline) are then summed up and displayed in PSF-CUSUM (Türker et al., 1996). The cumulative sum derived from the PSF provides information about even small changes between data points and is more robust to random fluctuations in counts (Davey et al., 1986).

We mainly used the PSF-CUSUM (Davey et al., 1986; Awiszus et al., 1991; Türker et al., 1994; Yavuz et al., 2015) during reflex responses, but PSTH to confirm, if inhibition, respectively excitation, occurs. In general we can say that PSF-CUSUM is constructed by MU discharging with a certain frequency, whereas the PSTH-CUSUM relies on the rhythmic discharge of MUs only; information retrieved from PSF-CUSUM is normalized to the number of stimulations received by the nerve (Yavuz et al., 2015).

The CUSUM in bin $i$ is expressed as following:

$$
\operatorname{CUSUM}_{i}=\sum_{j=1}^{j=i}\left(x_{j}-\bar{x}\right)
$$

where $x_{j}$ is the number of counts in bin $\mathrm{j}$ of PSTH, $\bar{x}$ is the mean value, calculated in a control period $u$ before the stimulus (Davey et al., 1986) (c.f. Figure 27). 


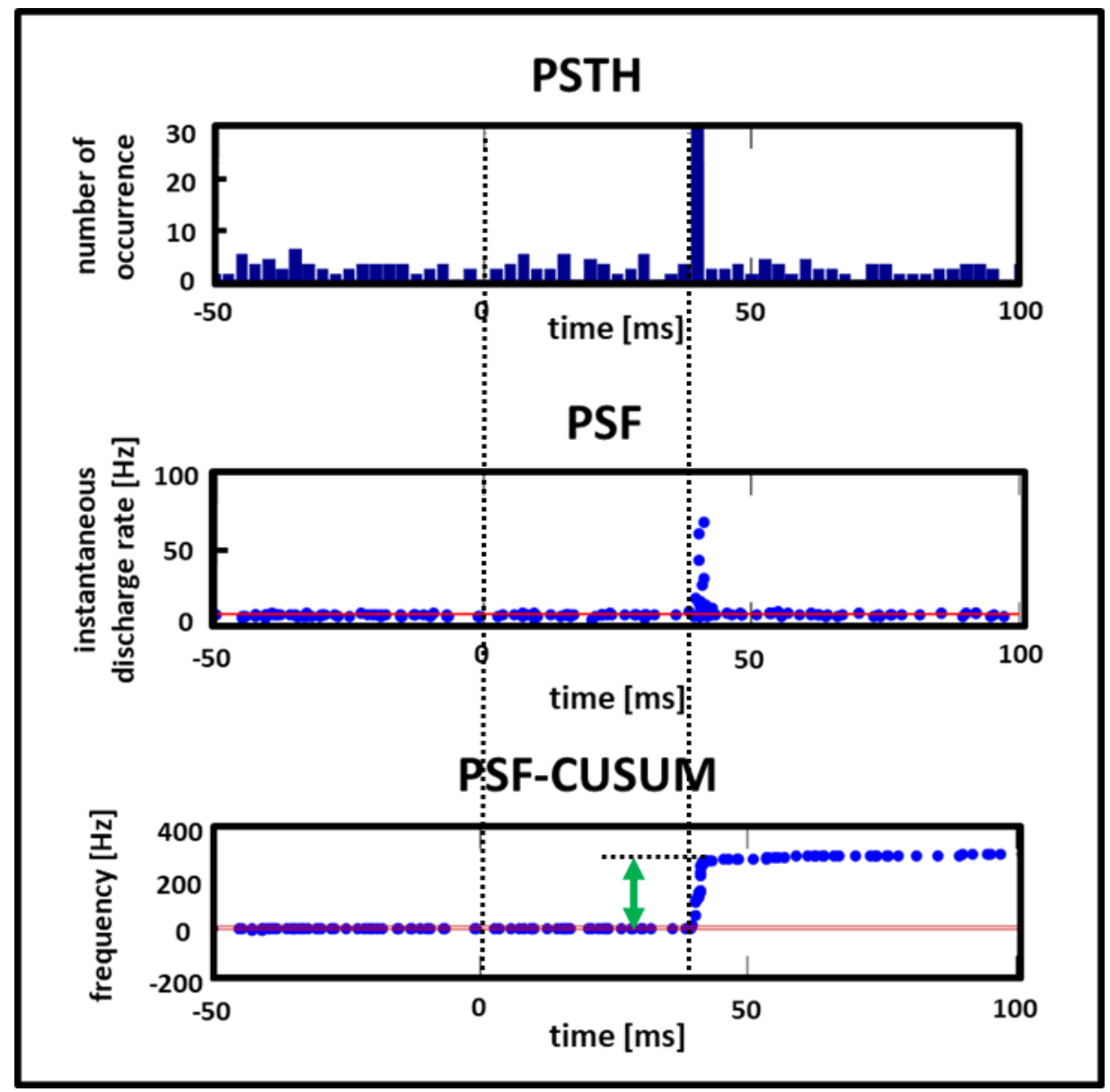

Figure 27: Explanation of data analysis (by reference to homonymous excitation) showing motor unit decomposition in peri-stimulus diagrams of three different types: PSTH, PSF and PSF-CUSUM; standard deviation shown as red lines in PSF and PSF-CUSUM; vertical dotted line represents trigger signal $(0 \mathrm{~ms}$ ) and onset of excitatory reflex (approx. $42 \mathrm{~ms}$ ); horizontal dotted line represents strength of excitatory reflex; green arrow depicts reflex amplitude [Hz].

\subsubsection{Estimation of recruitment threshold and reflex distribution}

Elicited reflexes in the homonymous muscle (c.f. Figure 27) as well as bisynaptic inhibition (Yavuz et al., 2018) in the antagonistic muscle, were investigated in a $400 \mathrm{~ms}$ frame, $200 \mathrm{~ms}$ before and after the stimulation, using peri-stimulus time histogram (PSTH) [count], peri-stimulus frequencygram (PSF) $[\mathrm{Hz}]$ and peri-stimulus frequencygram's cumulative sum (PSF-CUSUM) [Hz]. The PSTH shows the count of instantaneous discharge occurrence in a particular bin-width around the stimulus, whereas the PSF describes the instantaneous discharge rate around the stimulus (Awiszus et al., 1991; Türker et al., 1994); PSF is a more robust estimation of reflex responses (Yavuz et al., 2015) and PSTH was used to confirm the onset of either inhibitory or excitatory reflex.

In PSF-CUSUM, the difference between minimum and onset of reflex response was defined as strength of reciprocal inhibition. For H-reflex measurement in the homonymous muscle, the difference between minimum before and maximum after reflex-onset was defined as (excitatory) 
Introduction

reflex amplitude. Maximum PSF-CUSUM deviation from pre-stimulus time window was defined as significance of inhibition strength (Yavuz et al., 2018). Only those troughs that exceed significance thresholds were used to calculate excitatory and inhibitory reflex responses. Normalization to the number of stimulations gave information about extra discharge reduction per stimulation during the inhibitory protocol (Yavuz et al., 2018). Low-pass filtered rectified EMG provided us information about motor unit recruitment thresholds, whereas reflex distribution was estimated after finding the best fitting distribution for each muscle (normal, gamma, exponential), in accordance to the highest goodness of fit value (Yavuz et al., 2018).

The thresholds of recruited MUs define the amount of activation that is needed to recruit corresponding MUs during voluntary muscle contraction. It has been proposed that motor unit recruitment happens in size-dependent order: excitatory postsynaptic potentials (EPSP) result in depolarization, leading to recruitment of small-diameter motoneurons at first and large-diameter motoneurons at second (Henneman et al., 1965); this is thought to depend on the lower membrane potentials of small-diameter fibers compared to large-diameter fibers, resulting in higher thresholds for large-diameter MUs (Henneman et al., 1965; Henneman, 1977). The investigation of excitatory or inhibitory reflex inputs to the motoneuron pool depicts how this input is distributed for TA and TS muscles. Detailed information about the underlying procedure is shown in the subsequent chapter 2.9.4.

\subsubsection{Statistical analysis}

Study 1 (Yavuz et al., 2018): By normalization of H-reflex amplitudes to M-waves and comparison across subjects using t-test for normally distributed data, we checked whether the amount of reflex input was standardized. Low decomposition accuracy MUs (SIL < 0.9) (Negro et al., 2016), as well as MUs showing coefficient of variation of inter spike interval greater than $35 \%$ were excluded from the analysis. Mann-Whitney-U-test was used to estimate differences between reciprocal inhibition strength, recruitment thresholds across motor unit populations and reflex amplitudes since data was not normally distributed. The test compares interquartile ranges as well as median distribution. By usage of all trials ( 10 and $20 \%$ MVC dorsi-extension and plantar-flexion) probability density distributions of single motor unit responses were estimated. Estimation of bin size for probability density histogram was based on Freedman-Diaconis' rule and on interquartile ranges. The best fitting curve for each muscle among normal, gamma and exponential distribution, depending on the highest goodness of fit value, was selected. The goodness of fit value is defined by the discrepancy between distribution of empirical data and theoretical distribution model (D'Agostino et al., 1986). 
Introduction

Median, interquartile (Q1 - $1.5 *$ IQR) and absolute range of selected distributions between muscles were compared. Bivariate Spearman correlation analysis provided associations between motor unit recruitment threshold and reciprocal inhibition amplitude. To determine whether the portion of responsive motor units was different between muscles, since data were not normally distributed, modified t-test was used. A significance level of $p<0.05$ was chosen since no repetitive testing was performed in this study.

Study 2: Leaned on the statistical analyzes by Yavuz et al. (Yavuz et al., 2015, 2018), we investigated our data statistically. We checked normalized H-reflex amplitudes to M-waves across subjects (using t-test) to check whether the amount of excitatory reflex input was standardized. We excluded low decomposition accuracy MUs (SIL < 0.9). To analyze differences in reflex amplitudes between the group of skilled martial artists and control, we examined MUs of SOL and GM with Mann-Whitney-Utest, since data were not normally distributed. We compared median and interquartile (Q1 $1.5 *$ IQR) and absolute range of reflex amplitudes. To estimate differences in global EMG (sEMG) we compared median activity [mV] between groups in SOL and GM at $20 \% \mathrm{MVC}$. Investigation of recruitment thresholds was accomplished by assigning the first $5 \%$ of MVC as early recruited, while performing sustained ramp contraction, and the latest $5 \%$ before reaching the desired amount of force as lately recruited. By comparing the mean between groups, we analyzed differences in reflex amplitudes in dependency of recruitment thresholds.

Study 3: In this study, we decided to focus on analyses of average force output, mean MU discharge rate, stretching angle and global EMG (sEMG) activity. Due to sample size, t-test failed and we compared means for the above mentioned parameters. For the estimation of electromechanical delay (EMD) we used the time difference between so onset of M-wave and the exertion of force. For this purpose we averaged data from EMG and force around the stimulus ( $\pm 100 \mathrm{~ms}$ ). 


\section{Studies}

\subsection{Study 1 - Reciprocal Inhibition}

\section{Study 1 - Reciprocal inhibition}

$\frac{\text { Sustained }}{\text { plantar flexion }}$

$$
\frac{120^{\circ} \text { and } 170^{\circ}}{\text { knee angle; }}
$$

Sustained dorsal extension (TN stimulation)

\section{Preparation}

HDsEMG mounting (GM, SOL, TA)

MVC determination<smiles>C=CC</smiles>

Stimulation electrode mounting

M-Wave / H-Reflex tuning

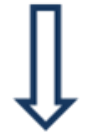

Reflex estimation

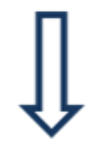

Data analysis

Figure 28: Flowchart showing most relevant stages during the experiment in study 1.

\subsubsection{Methods}

In this study we aimed to examine, with the technique of H-reflex, lower limb's muscle interplay in terms of reciprocal inhibition targeting TA and triceps surae muscle group.

Seven healthy, male subjects, age $25 \pm 4$ years, gave written informed consent according to the Declaration of Helsinki before the experiment started. The experimental study protocol was approved by the Human Ethics Committee of the University Medical Center, Georg-AugustUniversität Göttingen (approval number 1/10/12). 
Subjects were asked to perform the following procedure (c.f. Figure 28). They were seated on a chair of the Biodex System 3 Pro $^{\circledR}$. Their right leg was fixed with a fixing belt to the footplate. HDsEMG recording electrodes were mounted on $M$. gastrocnemius, $M$. soleus and $M$. tibialis anterior and stimulation electrode was either mounted on common peroneal nerve or, respectively, on tibial nerve. In different sessions we stimulated TN in the popliteal fossa (for reciprocal inhibition of TA) and CPN on the fibula's head (for reciprocal inhibition of triceps surae muscle group). The subjects' knee angle was $120^{\circ}$ for measurements of TA and SOL, and $170^{\circ}$ for GM measurement. Sessions were randomized. The participants were asked to perform sustained plantar flexion (for triceps surae reciprocal inhibition) and dorsal extension (for TA reciprocal inhibition). Recording parameters were set as described in Material and Methods 2.8.

To determine the subjects' maximum voluntary contraction force, we asked the subjects to perform three times plantar flexion, respectively dorsal extension, with their maximum amount of force. Resting interval between trials was $2 \mathrm{~min}$. As maximum voluntary contraction level for each participant the maximum peak of three consecutive contractions was chosen. Each subject performed randomly at $10 \% \mathrm{MVC}$, respectively $20 \%$, plantar flexion, respectively dorsal extension. While this, the innervating nerve of the antagonistic muscle was stimulated: while plantar flexion, CPN was selectively stimulated to elicit reflexes in TA; while dorsal extension, TN was selectively stimulated to elicit reflexes in Triceps surae muscles. M-wave/H-reflex tuning curve was recorded and provided us the correct stimulation intensities to elicit clear H-reflexes, free of M-wave, for reciprocal inhibition in the antagonistic muscle to follow. For standardization, stimulation intensities to elicit $\mathrm{H}$-reflexes with amplitude size of $10 \%$ of maximum $\mathrm{M}$-wave were set. After determining these stimulation intensities for each subject, a minimum of 150 pulses (square-wave pulse, 0.3 $0.5 \mathrm{~ms}$ pulse duration, $1-2 \mathrm{~s}$ random inter pulse interval) were applied for each sustained contraction level and for dorsal extension and plantar flexion. Each trial started with $30 \mathrm{~s}$ of ramp contraction, starting at zero amount of force and increasing to the double amount of according MVC force ( 20 and $40 \%$ ) and reducing again to the according amount of MVC force (10\% and $20 \%$ ) the trial is performed with.

\subsubsection{Results Study 1}

Mean stimulation intensities to elicit H-reflexes for TA were $10.3 \pm 1.9 \mathrm{~mA}$, for SOL $8.8 \pm 2.9 \mathrm{~mA}$. Trials contaminated with M-wave response were excluded from calculations. In total, $788 \mathrm{MU}$ were recorded in the three accessed muscles in seven subjects at two contraction forces, where the reflex 
amplitude in the reciprocal inhibition was significant for 376 of the identified MUs. T-test showed significantly higher responsiveness of MUs for both contraction levels $(p<0.05)$.
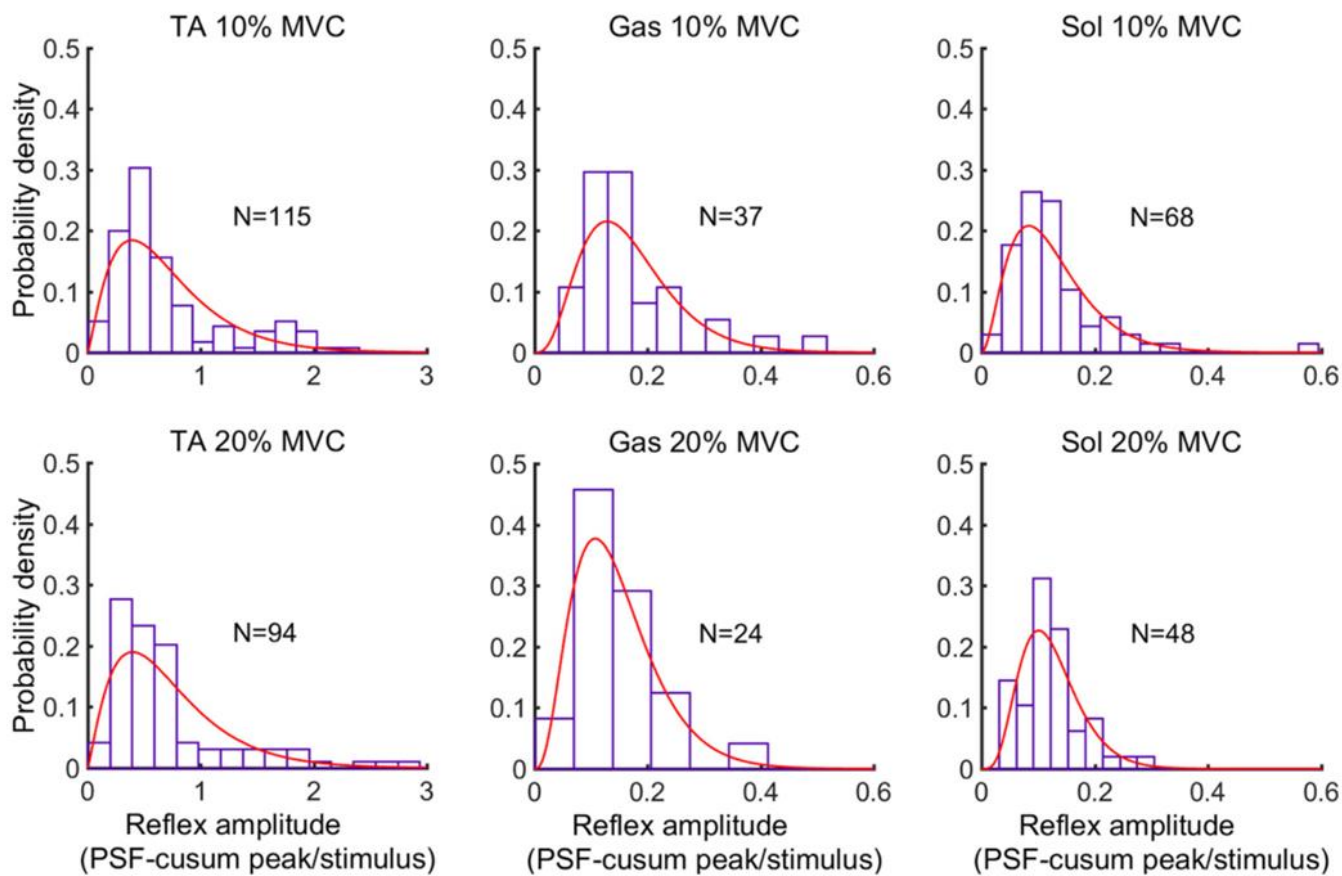

Figure 29: Empirical probability density histogram for reflex amplitudes of TA, GM and SOL with fitted gamma distribution for TA, GM and SOL at 10 and $20 \%$ MVC (by Yavuz et al., 2018); absolute range of probability density histograms $[\mathrm{Hz} /$ Stimuli] was approximately four times greater for TA.

Figure 29 shows empirical probability density histograms with fitted gamma distribution for TA, GM and SOL at 10 and $20 \%$ MVC. For TA the absolute range of probability density histograms was approximately four times greater $(0.13-2.33 \mathrm{~Hz} /$ stimulus and $0.09-2.75 \mathrm{~Hz} /$ stimulus) at $10 \%$ and $20 \% \mathrm{MVC}$, respectively) compared to SOL $(0.03-0.59 \mathrm{~Hz} /$ stimulus, $0.03-0.28 \mathrm{~Hz} /$ stimulus $)$ and GM (0.06- $0.48 \mathrm{~Hz} /$ stimulus, $0.4-0.38 \mathrm{~Hz} /$ stimulus).

In Figure $\mathbf{3 0}$ the cumulative probability curve for the targeted muscles are depicted. Median and range of reciprocal inhibition amplitude distribution was for both MVC levels greater for TA (MannWhitney-U-test), showing no significant differences between contraction force levels. 
A

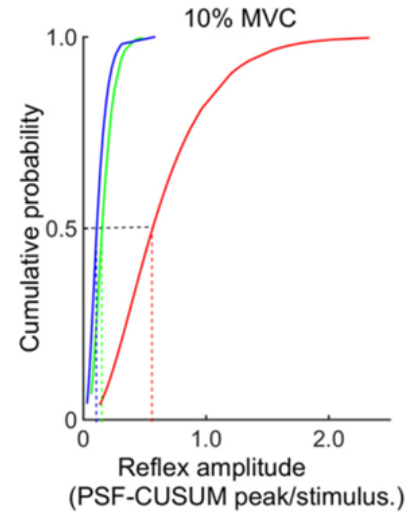

B

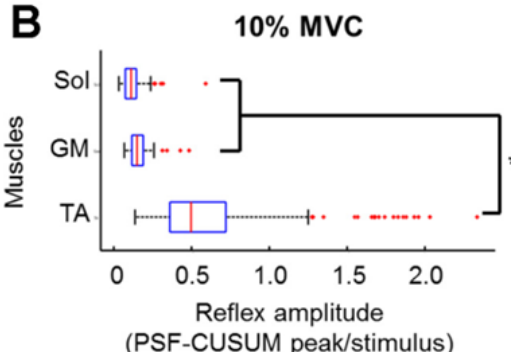

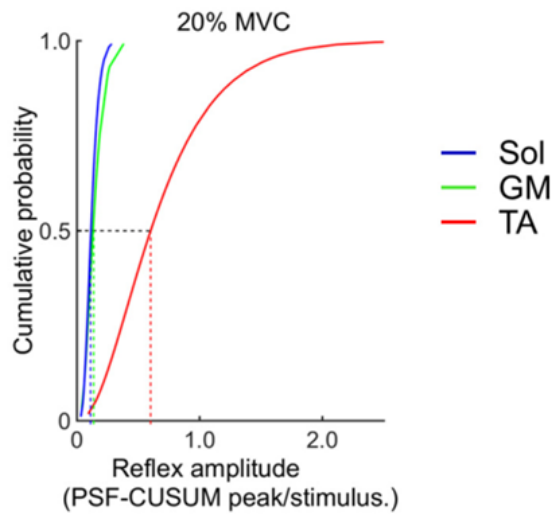

$20 \%$ MVC

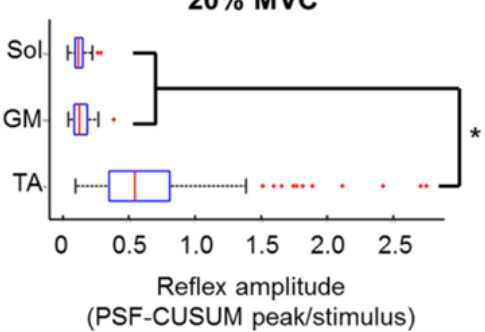

Figure 30: A: cumulative probability between distributions of reflex amplitudes; B: significance of reflex amplitudes through median and range values; median reflex amplitudes indicated by dashed vertical lines $(A)$ and red lines in box plots (B) (bv Yavuz et al.. 2018).

After normalization of the signal MU recruitment, thresholds were estimated from low-pass filtered rectified EMG. TA showed greater thresholds in $20 \% \mathrm{MVC}(13.5 \pm 4.4 \% \mathrm{MVC}$ for $\mathrm{GM}$, $14.9 \pm 1.1 \% \mathrm{MVC}$ for SOL and $18.8 \pm 7.2 \% \mathrm{MVC})$ compared to $10 \% \mathrm{MVC}(6.3 \pm 1.6 \% \mathrm{MVC}$ for GM, $7.6 \pm 1.1 \% \mathrm{MVC}$ for SOL and $7.1 \pm 2.1 \% \mathrm{MVC}$ for TA, $p>0.05)$.

Recruitment thresholds were used to calculate reflex amplitude distribution. We found that reflex amplitudes were negatively correlated with recruitment threshold for TA (bivariate Pearson correlation test, Pearson correlation value $\rho=-0.27$, significance $p=0.03)$. Whereas correlation was not significant for TA at $10 \%$ MVC, and for GM and SOL at 10 and $20 \%$ MVC ( $\rho$ : 0.14 for GM, 0.09 for SOL and -0.08 for TA at $10 \% \mathrm{MVC}$; 0.06 for GM, -0.11 for SOL at $20 \% \mathrm{MVC}$; $\mathrm{p}>0.05$ for all correlations); shown in Figure 31. 

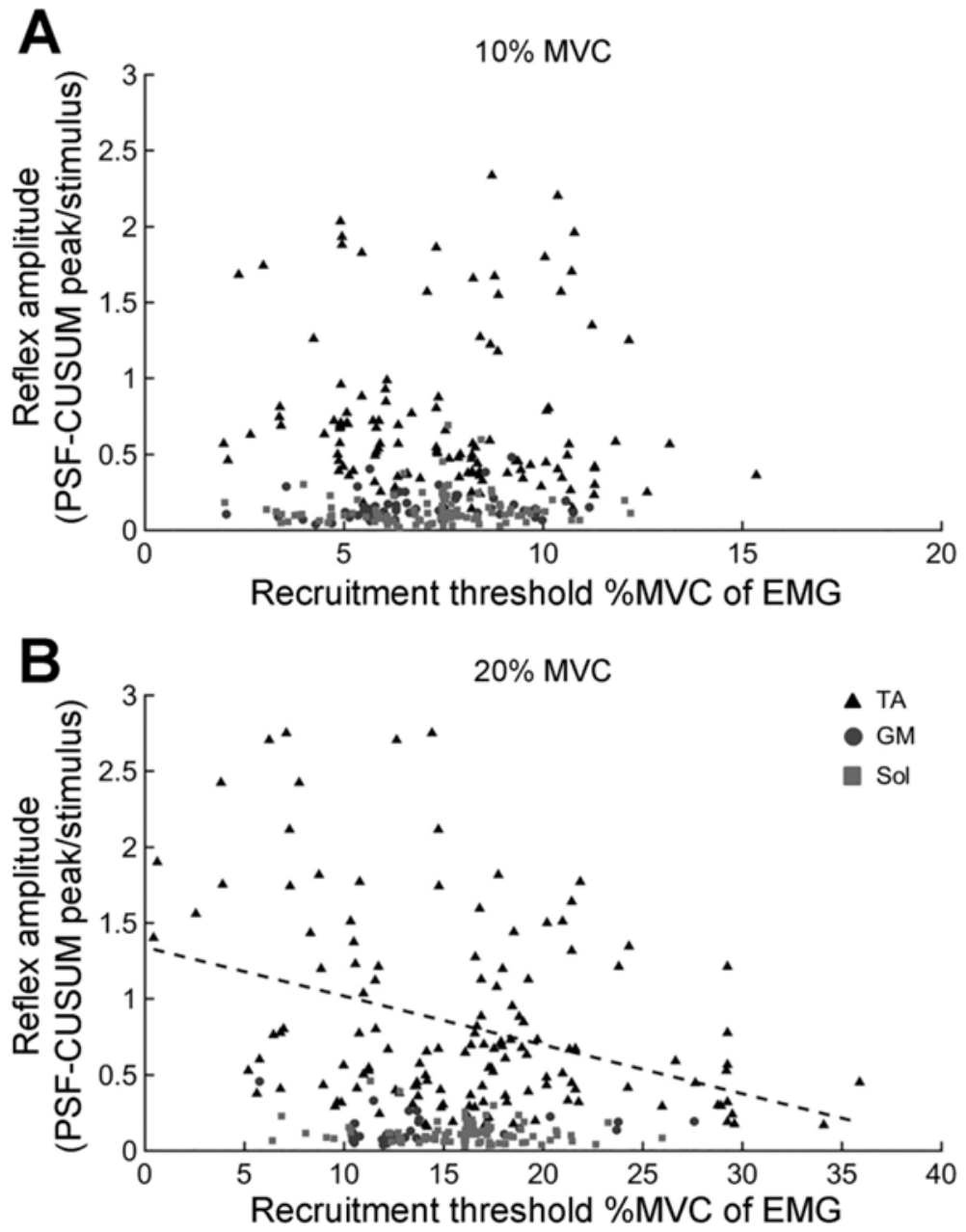

Figure 31: Correlation of recruitment thresholds used to calculate reflex amplitude (by Yavuz et al., 2018)

Reciprocal inhibitory response amplitudes for MUs with similar baseline DRs were compared to separate the effect of discharge rate (range $5-11 \mathrm{~Hz}$ for $10 \% \mathrm{MVC}$ and $5-13 \mathrm{~Hz}$ for $20 \% \mathrm{MVC}$ ) in all investigated muscles (at $10 \%$ MVC for $n_{T A}=100, n_{\text {SOL }}=68$ and $n_{G M}=37$; at $20 \% M V C$ for $n_{T A}=66$, $\mathrm{n}_{\mathrm{SOL}}=48$ and $\mathrm{n}_{\mathrm{GM}}=22$ ). Matt-Whitney-U test was used to calculate differences between groups, since normality test failed. TA $(0.45 \pm 0.24 \mathrm{~Hz} /$ stimulus, $0.49 \pm 0.31 \mathrm{~Hz} /$ stimulus at 10 and $20 \% \mathrm{MVC})$ motor units showed significantly $(\mathrm{p}<0.05)$ higher response compared to GM $(0.16 \pm 0.25 \mathrm{~Hz} /$ stimulus, $0.14 \pm 0.08 \mathrm{~Hz} /$ stimulus at 10 and $20 \% \mathrm{MVC}$ ) and SOL $(0.12 \pm 0.09 \mathrm{~Hz} /$ stimulus, $0.12 \pm 0.05 \mathrm{~Hz} /$ stimulus at 10 and $20 \% \mathrm{MVC})$; shown in Figure 32 . 

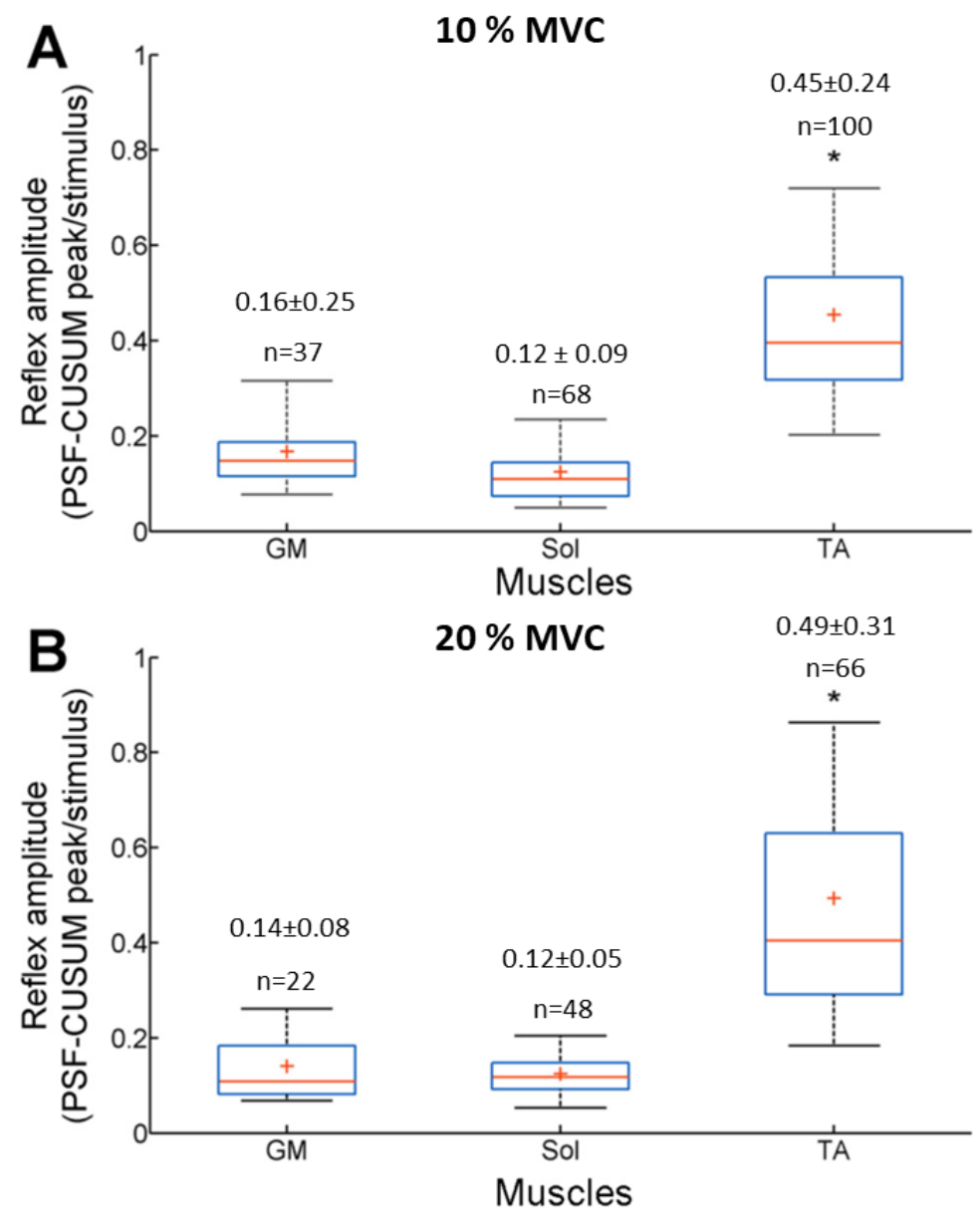

Figure 32: Reciprocal inhibitory reflex amplitudes [Hz/N.Stim] for GM, SOL and TA, by Yavuz et al., 2018, modified; red lines indicate median reflex amplitudes, + indicates the average reflex amplitudes.

\subsubsection{Author contributions}

Published paper: Utku S. Yavuz, Francesco Negro and Dario Farina conceived and designed experiment and research. Experiments were performed by Utku S. Yavuz, Francesco Negro and Robin Diedrichs. Utku S. Yavuz and Robin Diedrichs analyzed data. Those data were interpreted by Utku S. Yavuz, Francesco Negro and Dario Farina. Figures were prepared by Utku S. Yavuz (modified for this thesis by Robin Diedrichs). Figure 28 prepared by Robin Diedrichs. The manuscript was created by Utku S. Yavuz, Francesco Negro and Dario Farina (discussion for this thesis by Robin Diedrichs). All authors edited and revised the full manuscript and all approved final version of manuscript. Robin Diedrichs joined the lab for his PhD thesis while this study was conducted. 
Study 2 - Homonymous Excitation

$\begin{array}{lcl}\frac{120^{\circ} \text { and } 170^{\circ}}{\text { plantar flexion }} & \frac{\text { Skilled group and }}{\text { knee angle; }} & \text { control group } \\ \text { (TN stimulation) } & 10 \text { and } 20 \% \mathrm{MVC} & \end{array}$

Preparation

HDsEMG mounting (GM, SOL, TA)

MVC determination<smiles>[C]=C</smiles>

Stimulation electrode mounting M-Wave / H-Reflex tuning

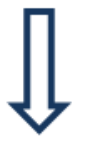

Reflex estimation

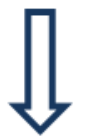

Data analysis

Figure 33: Flowchart showing most relevant stages during the experiment in study 2.

\subsubsection{Methods}

The subjects defined as skilled group, were chosen according to their experience and regular training activity in the martial art of Ninjutsu. Skilled subjects were moderately athletic, participated and trained at least two times a week and participated in training for at least three years and wear a black belt. Control group subjects were moderately active but without regular engaging in sports. Pooled subjects' age was $31 \pm 7$ years. All subjects gave written informed consent according to the Declaration of Helsinki before the experiment started. The experimental study protocol was approved by the Human Ethics Committee of the University Medical Center, Georg-AugustUniversität Göttingen (approval number 24/2/16). 
The subjects were seated in a chair of the Biodex System 3 Pro ${ }^{\circledR}$. Subjects were asked to perform the following procedure (c.f. Figure 33). Right leg was fixed with a fixing belt to the footplate. HDsEMG recording electrodes were mounted on SOL and TA, stimulation electrode was mounted on TN. We stimulated TN in the popliteal fossa for excitation of the homonymous muscle. The subjects' knee angle was $120^{\circ}$ for measurement of SOL, for GM measurement an angle of $170^{\circ}$ was set (knee not entirely straight, not locked). Sessions were randomized. The participants were asked to perform sustained plantar flexion for the estimation of $\mathrm{H}$-reflexes in the homonymous muscle. Recording parameters were set as described in Material and Methods 2.8; resting periods between trials were at least $120 \mathrm{~s}$. Determination of the subjects' maximum voluntary contraction force was performed as described for study 1 in chapter 3.1.1. . Each trial started with $30 \mathrm{~s}$ of ramp contraction, starting at zero amount of force and increasing to the double amount of according MVC force and reducing again to the according amount of MVC force $(10 \% \rightarrow 20 \% \rightarrow 10 \%$ MVC and $20 \% \rightarrow 40 \% \rightarrow 20 \%$ MVC) the trial is performed with. Then a minimum of 150 pulses (square-wave pulse, $0.3-0.5 \mathrm{~ms}$ pulse duration, 1 - $2 \mathrm{~s}$ random inter pulse interval) were applied at each contraction level. Sessions were randomized; resting period in between trials was $300 \mathrm{~s}$.

\subsubsection{Results Study 2}

The applied mean stimulation intensities to elicit $\mathrm{H}$-reflex within both groups were 12.0 $\mathrm{mA} \pm 5.1 \mathrm{~mA}$. Trials with $\mathrm{M}$-wave response were excluded from further calculations.

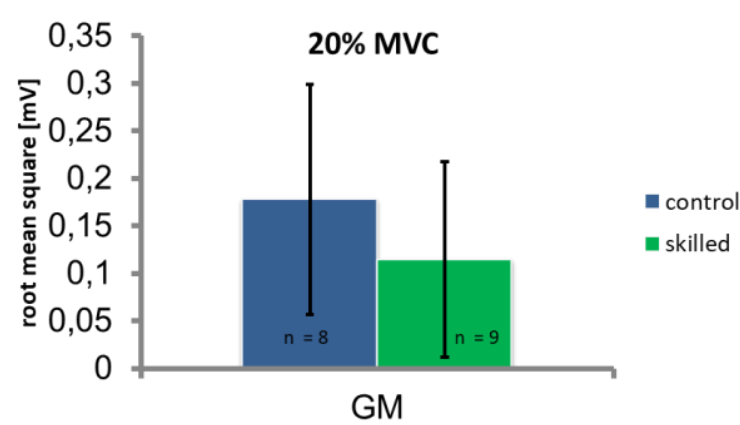

Figure 34: Median root mean square (RMS) $[\mathrm{mV}]$ from $\mathrm{GM}$ at $20 \%$ MVC during sustained contraction

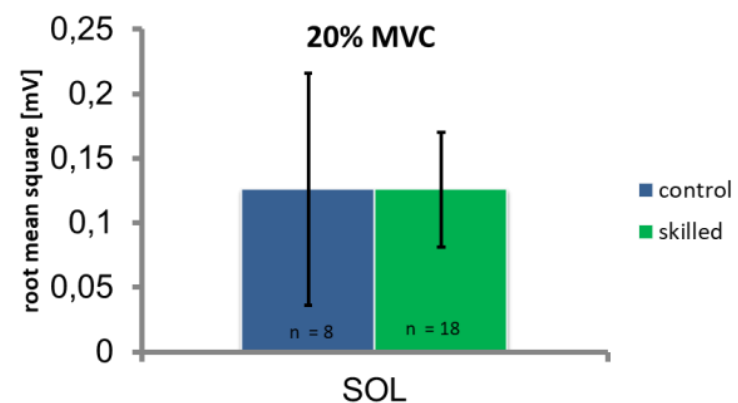

Figure 35: Median root mean square (RMS) [mV] from SOL at $20 \%$ MVC during sustained contraction

Table 4: Root mean square (RMS) $[\mathrm{mV}]$ and median from GM and SOL at $20 \% \mathrm{MVC}$ during sustained contraction:

\begin{tabular}{|c|c|c|c|c|}
\hline Group & $\begin{array}{c}\text { GM RMS } \pm \text { STDEV } \\
{[\mathrm{mV}]}\end{array}$ & $\begin{array}{c}\text { SOL RMS } \pm \text { STDEV } \\
{[\mathrm{mV}]}\end{array}$ & $\begin{array}{c}\text { GM median RMS } \\
{[\mathrm{mV}]}\end{array}$ & $\begin{array}{c}\text { SOL median RMS } \\
{[\mathrm{mV}]}\end{array}$ \\
\hline Control & $0.187 \pm 0.121$ & $0.146 \pm 0.090$ & $0.178 \pm 0.123$ & $0.126 \pm 0.094$ \\
\hline Skilled & $0.156 \pm 0.103$ & $0.125 \pm 0.044$ & $0.115 \pm 0.101$ & $0.126 \pm 0.040$ \\
\hline
\end{tabular}


We analyzed the root mean RMS (Hug et al., 2006; Fukuda et al., 2010) at $20 \%$ MVC during ramp part of SEMG by digitally converting the HDsEMG electrode into a bipolar sEMG electrode using Laplace operator. Calculated median RMS does not differ significantly between groups while subjects perform sustained plantar flexion with $20 \% \mathrm{MVC}$ (control $\mathrm{GM}_{\mathrm{M}}=0.18 \pm 0.12 \mathrm{mV}$, skilled $_{\mathrm{GM}}=0.13 \pm 0.09 \mathrm{mV}$, control $\mathrm{SOL}=0.12 \pm 0.10 \mathrm{mV}$, skilled $\mathrm{SOL}=0.13 \pm 0.04 \mathrm{mV}$ ), results for $\mathrm{GM}$ are plotted in Figure 34, respectively for SOL in Figure 35. Data were acquired from SOL and GM $\left(n_{\text {skilled }}=8 ; n_{\text {skilled }}=9\right)$. Differences at $10 \%$ MVC are not shown since data acquisition was not sufficient to provide statistically significant results.

Further investigating neural properties, we estimated reflex amplitudes at $10 \%$ and $20 \%$ MVC while subjects performed sustained plantar flexion (Figure $\mathbf{3 6}$ and Figure 37). Since normality test failed, we used Mann-Whitney-U test. It confirmed significant differences in the size of reflex amplitudes between both groups in both corresponding muscles $(p<0.05)$.

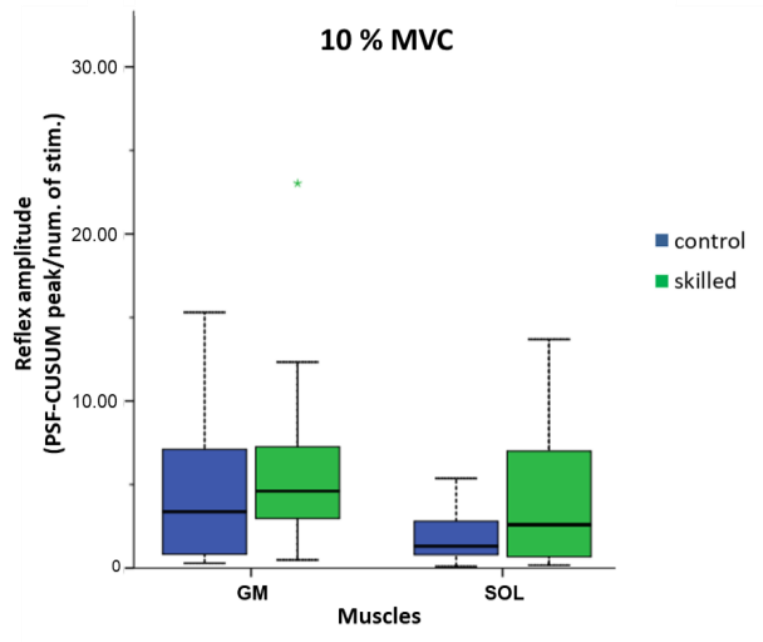

Figure 36: Reflex amplitudes (PSF-CUSUM peak/num. of. stim.), $10 \%$ MVC for GM and SOL, $p<0.05$ for all medians within muscle groups, * depicts outlier.

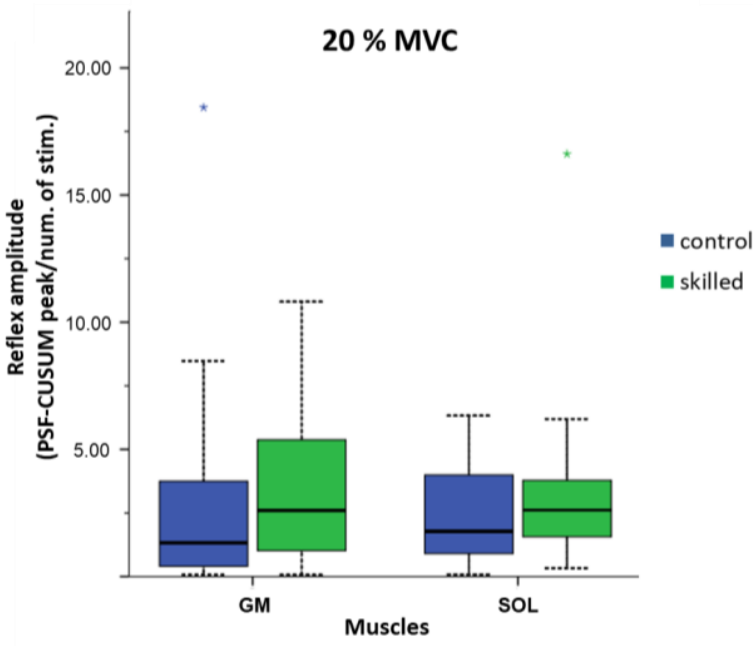

Figure 37: Reflex amplitudes (PSF-CUSUM peak/num. of. stim.), $20 \%$ MVC for GM and SOL, $p<0.05$ for all medians within muscle groups, * depicts outlier.

In total we detected $743 \mathrm{MUs}$ (499 in SOL and 244 in GM). Median reflex amplitudes with median absolute deviation were significantly different $(p<0.05)$ for GM and SOL at $10 \%$ and $20 \%$ MVC (

Table 5).

Table 5: Values of boxplot of reflex amplitudes (PSF-CUSUM peak/num. of. stim.) at $10 \%$ and $20 \%$ MVC for GM and SOL: 


\begin{tabular}{|c|c|c|c|c|c|c|}
\hline & Muscle & Group & $\begin{array}{c}\text { Mean reflex } \\
\text { amplitude } \pm S T D \\
\end{array}$ & $\begin{array}{l}\text { Median reflex } \\
\text { amplitude } \pm \text { MAA }\end{array}$ & Quartile $25^{\text {th }}$ & Quartile $75^{\text {th }}$ \\
\hline \multirow{4}{*}{ MVC10 } & \multirow{2}{*}{ GM } & Control & $4.31 \pm 3.86$ & $3.38 \pm 0.01$ & 0.821 & 7.121 \\
\hline & & Skilled & $5.15 \pm 3.67$ & $4.61 \pm 0.01$ & 2.918 & 7.261 \\
\hline & \multirow{2}{*}{ SOL } & Control & $2.07 \pm 1.88$ & $1.31 \pm 0.01$ & 0.772 & 2.963 \\
\hline & & Skilled & $4.33 \pm 4.98$ & $2.59 \pm 0.02$ & 0.660 & 7.033 \\
\hline \multirow{4}{*}{ MVC 20} & \multirow{2}{*}{ GM } & Control & $2.31 \pm 2.35$ & $1.33 \pm 0.01$ & 0.365 & 3.372 \\
\hline & & Skilled & $3.38 \pm 1.87$ & $2.60 \pm 0.01$ & 0.957 & 5.204 \\
\hline & \multirow{2}{*}{ SOL } & Control & $2.39 \pm 2.88$ & $1.78 \pm 0.04$ & 0.884 & 3.443 \\
\hline & & Skilled & $2.72 \pm 1.43$ & $2.61 \pm 0.01$ & 1.534 & 3.262 \\
\hline
\end{tabular}

Further examination of data showed differences in the recruitment thresholds of the previous investigated motor units. These differences are not significant, since not all previous recorded reflex amplitudes of motor units possessed matching recruitment thresholds. Overall, recruitment thresholds of $n=61$ in the control and $n=93$ in the skilled group motor units were measured. Results are shown in Figure 38 and Figure 39. In general, columns indicate that skilled participants perform with higher reflex amplitudes in SOL muscle according to early (first $5 \%$ of MVC) and late (amount of MVC during experiment minus $5 \%$ ) recruited MUs at $10 \%$ and $20 \%$ MVC. Mean reflex amplitudes were not significantly different for early recruited MUs at SOL at $10 \% \mathrm{MVC}$ (control early: $_{\text {: }}$ : $5.85 \pm 0.59 \mathrm{~Hz} /$ num. of stim., skilled early: $7.27 \pm 1.10 \mathrm{~Hz} /$ num. of stim.) and late recruited MUs at $10 \%$ MVC (control late: $7.57 \pm 0.73 \mathrm{~Hz} / \mathrm{N}$.Stim., skilled late: $8.12 \pm 1.42 \mathrm{~Hz} /$ num. of stim.). Results are also insignificant for early recruited SOL MUs at $20 \%$ MVC (control $l_{\text {early: }} 15.29 \pm 1.04 \mathrm{~Hz} /$ num. of stim., skilled $_{\text {early: }}: 16.75 \pm 0.94 \mathrm{~Hz} / \mathrm{N}$.Stim.) and late recruited MUs at $20 \% \mathrm{MVC}$ (control late: $13.54 \pm 2.32$ $\mathrm{Hz} /$ num. of stim.., skilled $\mathrm{late}_{\mathrm{ate}}: 20.37 \pm 2.53 \mathrm{~Hz} /$ num. of stim.). 


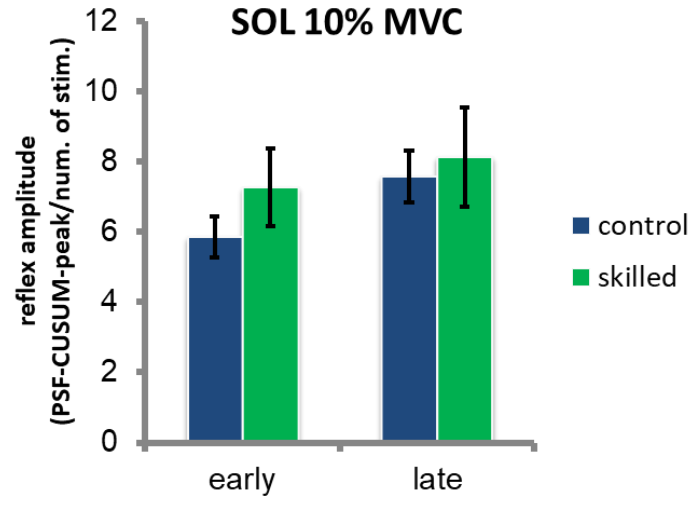

Figure 38:Mean reflex amplitudes (PSF-CUSUMpeak/num. of stim.) of early and lately recruited MUs at $10 \%$ MVC from SOL

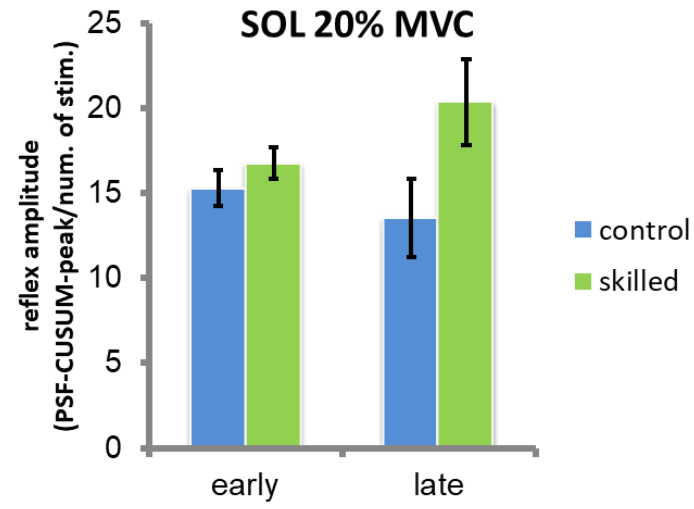

Figure 39: Mean reflex amplitudes (PSF-CUSUMpeak/num. of stim.) of early and lately recruited MUs at $20 \%$ MVC from SOL

\section{MVC $10 \%$}

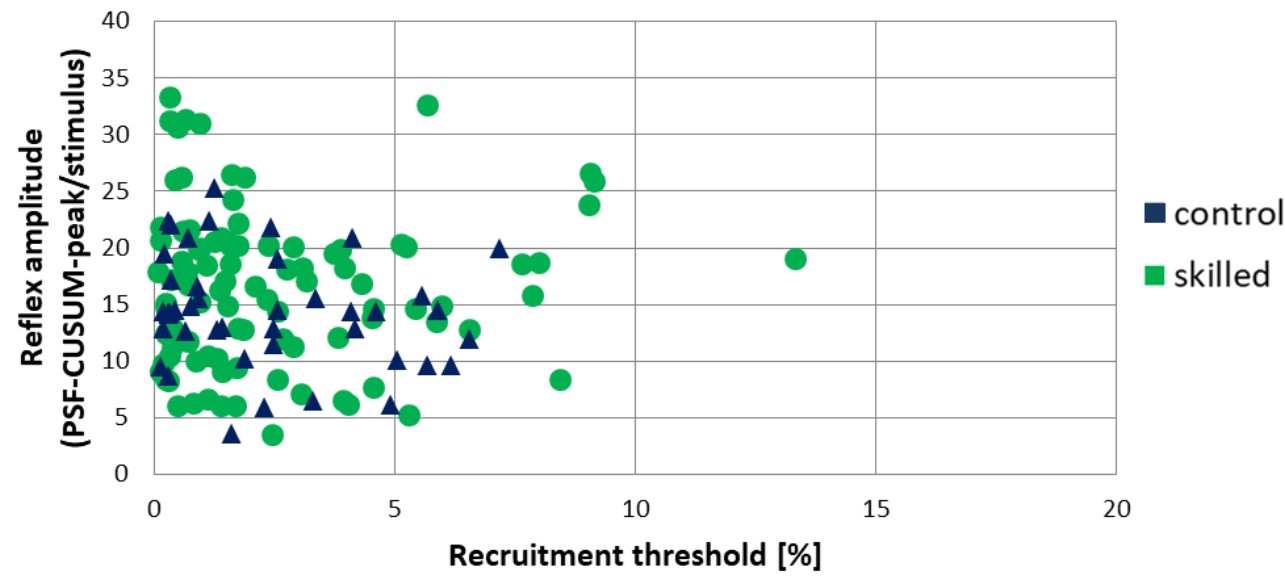

Figure 40: Scatter-plot of reflex amplitudes as a function of recruitment threshold of all recruited and analyzed motor units during ramp part at $10 \% \mathrm{MVC}, \mathrm{n}_{\text {control }}=69, \mathrm{n}_{\text {skilled }}=76$.

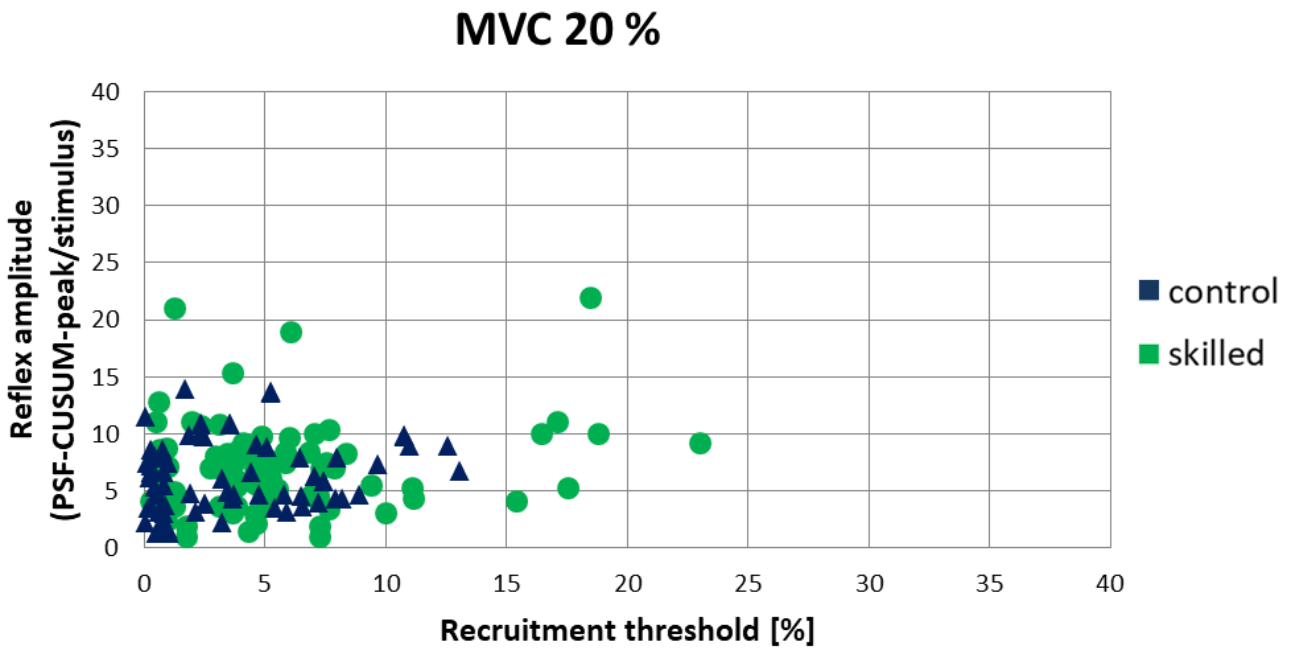

Figure 41: : Scatter-plot of reflex amplitudes as a function of recruitment threshold of all recruited and analyzed motor units during ramp part at $20 \% M V C, n_{\text {control }}=98, n_{\text {skilled }}=50$. 
Figure $\mathbf{4 0}$ and Figure $\mathbf{4 1}$ correlate with the distribution of reflex amplitudes in Figure $\mathbf{3 8}$ and Figure 39. Linear regressions are not plotted since differences are not significant:

$10 \%$ MVC: $\rho_{\text {control: }}-0.450 x \pm 15.469, R_{\text {control }}^{2}=0.0374, \rho_{\text {skilled }}: 0.158 x \pm 15.889, R_{\text {skilled }}=0.0036$;

$20 \%$ MVC: $\rho_{\text {control: }} 0.1326 x \pm 5.9932, R_{\text {control }}^{2}=0.0217, \rho_{\text {skilled }}: 0.1266 x \pm 6.3426, R_{\text {skilled }}^{2}=0.0 .0229$

\subsubsection{Author contributions}

Robin Diedrichs, Utku S. Yavuz and Dario Farina conceived and designed experiment and research. Experiments were performed by Robin Diedrichs and Utku S. Yavuz. Robin Diedrichs and Utku S. Yavuz analyzed data. Those data were interpreted by Robin Diedrichs and Utku S. Yavuz Dario Farina. Figures 33 - 37 were prepared by Robin Diedrichs, figures 38-41 by Robin Diedrichs with support of Utku S. Yavuz. The manuscript was created by Robin Diedrichs. 


\title{
Study 3 - Homonymous Excitation
}

$\begin{array}{cc}\frac{\text { Sustained }}{\text { plantar flexion }} & \text { Prone } \\ \text { (TN stimulation) } & \text { position }\end{array}$

Skilled group and control group

\author{
Preparation \\ HDsEMG mounting (GM, SOL, TA) \\ MVC determination
}<smiles>C=CC</smiles>

Stimulation electrode mounting M-Wave / H-Reflex tuning<smiles>[C]1C=C1</smiles>

Pre-test: EMD, angle, force, discharge rate

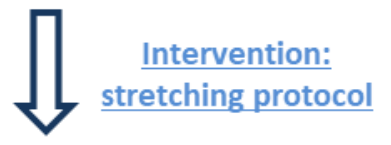

Post-test: EMD, angle, force, discharge rate<smiles>C=CC=C</smiles>

Data analysis

Figure 42: Flowchart showing most relevant stages during the experiment in study 3.

\subsubsection{Methods}

Figure 43: Flowchart showing most relevant stages during the experiment in study 3.

Figure 22 depicts a common stretching technique in lyengar Yoga, called Supta-Pādāngushthāsana. The subjects in the skilled group were chosen according to their experience and regular training activity in Yoga. Skilled subjects were moderately athletic, participated and trained Yoga at least two times a week and participated in training regularly in the last 12 months. Control group subjects were moderately active but without regular engaging in sports, especially Yoga and other stretching exercises. Pooled subjects' age was $30 \pm 5$ years, gender was female only. All subjects gave written 
informed consent according to the Declaration of Helsinki before the experiment started. The experimental study protocol was approved by the Human Ethics Committee of the University Medical Center, Georg-August-Universität Göttingen (approval number 21/4/16). Results were presented in a Master thesis (Pröttel, 2017) but re-calculated for this PhD thesis.

Flowchart (Figure 43) shows the scheduled protocol, subjects were asked to perform. In this study, subjects were laid on a lounger in prone position attached to Biodex System 3 Pro $^{\circledR}$. Left leg was fixed with a fixing belt on the footplate during recordings and released for stretching protocol. HDsEMG recording electrodes were mounted on GM and TA; stimulation electrode was mounted on tibial nerve. We stimulated TN in the popliteal fossa for excitation of the homonymous muscle. The subjects' knee angle in prone position was between $170^{\circ}$ and $180^{\circ}$. The participants were asked to perform sustained plantar flexion; homonymous muscle was stimulated, for the estimation of $\mathrm{H}$ reflexes in the homonymous muscle. Recording parameters were set as described in Material and Methods. Determination of the subjects' maximum voluntary contraction force was performed as described for study 1 and study 2 in chapter 3.1.1.

After determining these stimulation intensities for each subject, they performed a pre-test with two trials at $50 \% \mathrm{MVC}$ without stimulations for $60 \mathrm{~s}$ each, followed by a break of $60 \mathrm{~s}$ as well as between and after trials. Sequent maximum M-waves were elicited and recorded (10 pulses) followed by $300 \mathrm{~s}$ of rest. Sequent a minimum of 150 pulses (square-wave pulse, $0.3-0.5$ ms pulse duration, 1 $2 \mathrm{~s}$ random inter pulse interval) were applied at $20 \%$ MVC. Each trial started with $30 \mathrm{~s}$ of ramp contraction, beginning at zero amount of force and increasing to the double amount of according MVC force (40\%) and reducing again to the according amount of MVC force $(20 \%)$ the trial is performed with. This is followed by an intervention, i.e. stretching protocol. Subjects were disengaged of the Biodex System, moved to supine position and were asked to perform $45 \mathrm{~s}$ of stretching at $10^{\circ}$ hip angle, followed by $60 \mathrm{~s}$ break. After this, subjects performed $45^{\circ}$ stretch, followed by $60 \mathrm{~s}$ of break. After this subjects moved to their individual maximum stretch angle, followed by $60 \mathrm{~s}$ of break. After this, subjects performed maximum stretch for $45 \mathrm{~s}$, directly followed by $15 \mathrm{~s}$ of absolute maximum stretch briefly beyond the angle subjects performed stretching before.

This procedure is followed by a post-test which is analogous to the pre-test.

\subsubsection{Results Study 3}

The applied mean stimulation intensities within both groups were $16.0 \mathrm{~mA} \pm 8.7 \mathrm{~mA}$. Since H-reflex recordings were contaminated with M-waves, no further estimation of motor units was performed. 


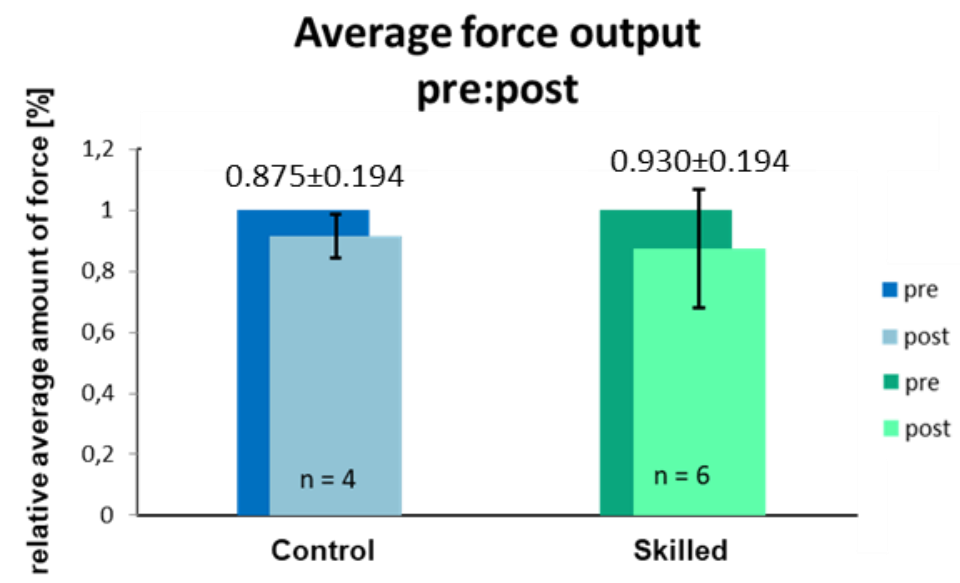

Figure 44 : Median relative force output comparing pre- and post-test in control and skilled group, pre-test set as 1.

The average force output before the stretching protocol was set to 1.00. After performing the stretching protocol, the average median force exertion of control group was $0.916 \pm 0.071$ and average mean force exertion $0.930 \pm 0.071(n=4)$. Skilled performed with an average median exertion force $0.875 \pm 0.194$ and average mean exertion force $0.930 \pm 0.194(n=6)$ (Figure 44).

\section{Mean motor unit discharge rate root mean square (RMS)}

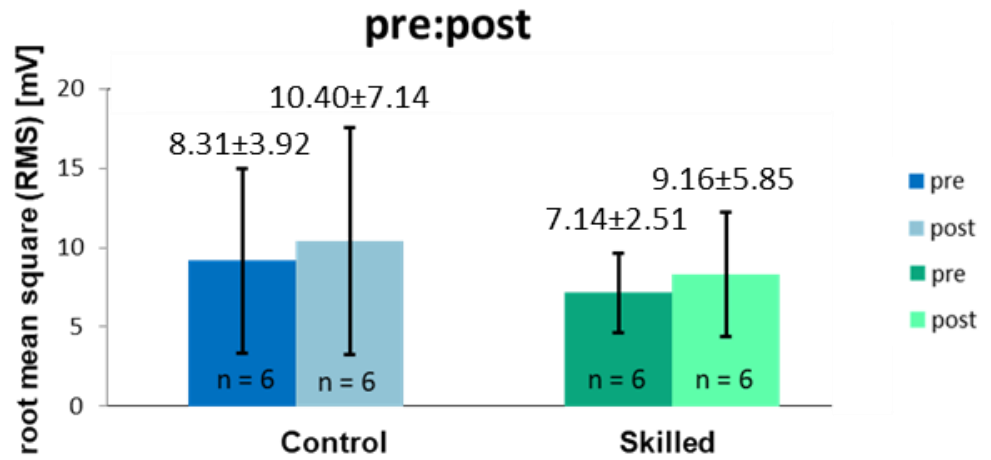

Figure 45: Mean motor unit discharge rate in RMS [mV] for pre- and post-test at $20 \% \mathrm{MVC}$ for control and skilled in SOL.

Table 6: Mean motor unit discharge rate in RMS [mV] for pre- and post-test at $20 \% \mathrm{MVC}$ for control and skilled in SOL:

\begin{tabular}{|c|c|c|c|}
\hline & Group & $\begin{array}{c}\text { Mean MUDR } \\
\text { RMS } \pm \text { STDEV [mV] } \\
\text { pre }\end{array}$ & $\begin{array}{c}\text { Mean MUDR } \\
\text { RMS } \pm S T D E V ~[m V] \\
\text { post }\end{array}$ \\
\hline \multirow{2}{*}{ MUDR \pm STDEV } & Control & $8.317 \pm 3.927$ & $10.405 \pm 7.142$ \\
\cline { 2 - 4 } & Skilled & $7.145 \pm 2.519$ & $9.162 \pm 5.849$ \\
\hline \multicolumn{3}{|c|}{} \\
\hline \multirow{2}{*}{ Count of MUs } & Control & 256 & 278 \\
\cline { 2 - 4 } & Skilled & 212 & 291 \\
\hline
\end{tabular}


Overall, we measured and evaluated 1037 single motor units and calculated mean motor unit discharge rate RMS during sustained plantar flexion (Table 6 and Figure 45). The mean motor unit discharge rate RMS in the control group before the stretching protocol was $\mathrm{mdr}_{\text {pre }}=8.31 \pm 3.93 \mathrm{mV}$ ( $n=6,256$ MUs) and after the protocol $\mathrm{mdr}_{\text {post }}=10.41 \pm 7.14 \mathrm{mV}(\mathrm{n}=6 ; 278 \mathrm{MUs})$. For skilled participants, mean motor unit discharge rate RMS before the protocol was measured as $\mathrm{mdr}_{\text {pre }}=7$. $15 \pm 2.52 \mathrm{mV}(\mathrm{n}=6,212 \mathrm{MUs})$ and after the protocol $\mathrm{mdr}_{\text {post }}=9.162 .41 \pm 5.85 \mathrm{mV}(\mathrm{n}=6 ; 291 \mathrm{MUs})$.

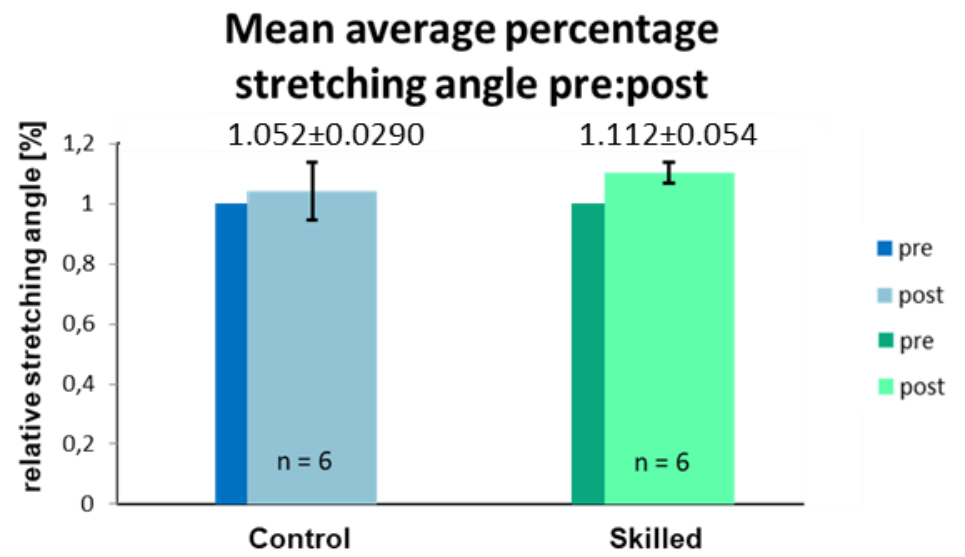

Figure 46: Mean average change in stretching angle, pre-test set as 1.

Table 7: Exemplary data of one subject showing mean average change in stretching angle:

\begin{tabular}{|l|c|c|c|c|c|}
\hline & $\operatorname{Max}_{1}\left[^{\circ}\right]$ & $\operatorname{Max}_{2}\left[^{\circ}\right]$ & $\left.\operatorname{Max}_{\text {plus }}{ }^{\circ}{ }^{\circ}\right]$ & $\begin{array}{c}\text { Resulting change } \\
\operatorname{Max}_{\text {plus }}-\operatorname{Max}_{1}\left[^{\circ}\right]\end{array}$ & $\begin{array}{c}\text { Resulting change } \\
\operatorname{Max}_{\text {plus }} / \operatorname{Max}_{1}[\%]\end{array}$ \\
\hline Subject & 101 & 100 & 119 & 18 & 117.8 \\
\hline
\end{tabular}

The average stretching angle before the stretching protocol was set to 1.00 . After performing the stretching protocol, the mean stretching angle of control group was $1.052 \pm 0.0290(n=6)$; skilled performed with $1.112 \pm 0.054(n=6)$ (Table 7 and Figure 46). 
Median electromechanical delay (EMD) pre:post

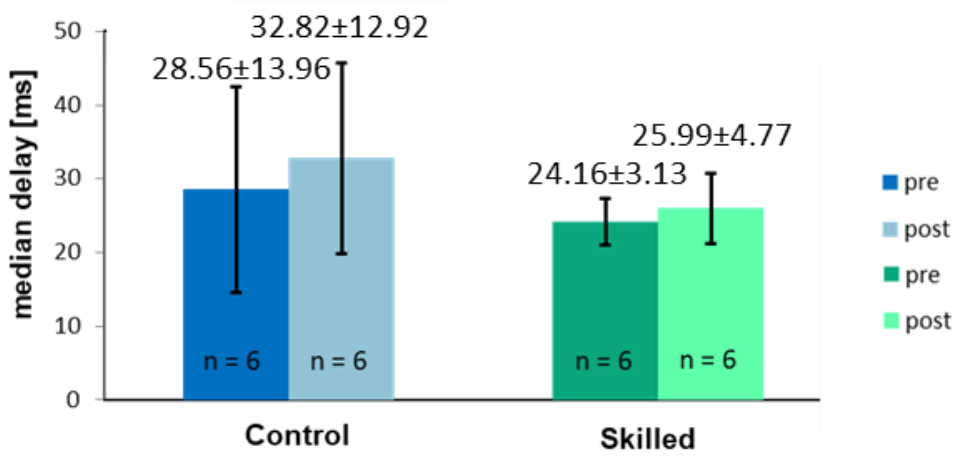

Figure 47: Median global EMG activity [mV] for pre- and post-test at $50 \% \mathrm{MVC}$ for control and skilled in SOL.

Table 8: Mean and median of electromechanical delay [ms] for pre- and post-test at $20 \% \mathrm{MVC}$ for control and skilled in SOL:

\begin{tabular}{|c|c|c|c|c|}
\hline Group & $\begin{array}{c}\text { Mean EMD } \\
\text { [ms] } \\
\text { pre }\end{array}$ & $\begin{array}{c}\text { Mean EMD } \\
\text { [ms] } \\
\text { post }\end{array}$ & $\begin{array}{c}\text { Median } \\
\text { EMD } \pm S T D E V \\
\text { [ms] } \\
\text { pre }\end{array}$ & $\begin{array}{c}\text { Median } \\
\text { EMD } \pm S T D E V \\
\text { [ms] } \\
\text { post }\end{array}$ \\
\hline Control & 36.338 & 35.900 & $28.567 \pm 13.962$ & $32.827 \pm 12.926$ \\
\hline Skilled & 24.410 & 25.102 & $24.164 \pm 3.133$ & $25.994 \pm 4.778$ \\
\hline
\end{tabular}

EMD calculated from M-wave recordings showed no significant differences for control and skilled participants comparing pre- with post-test Table 8 and Figure 47). Control showed in pre-test a median delay of $\mathrm{EMD}_{\text {pre }}=28.57 \pm 13.96 \mathrm{~ms}$ and in post-test $\mathrm{EMD}_{\text {post }}=32.83 \pm 12.93 \mathrm{~ms}$. Skilled performed in pre-test with a mean delay of $E M D_{\text {pre }}=24.16 \pm 3.13 \mathrm{~ms}$ and in post-test $E M D_{\text {post }}=26.00 \pm 4.78 \mathrm{~ms}$.

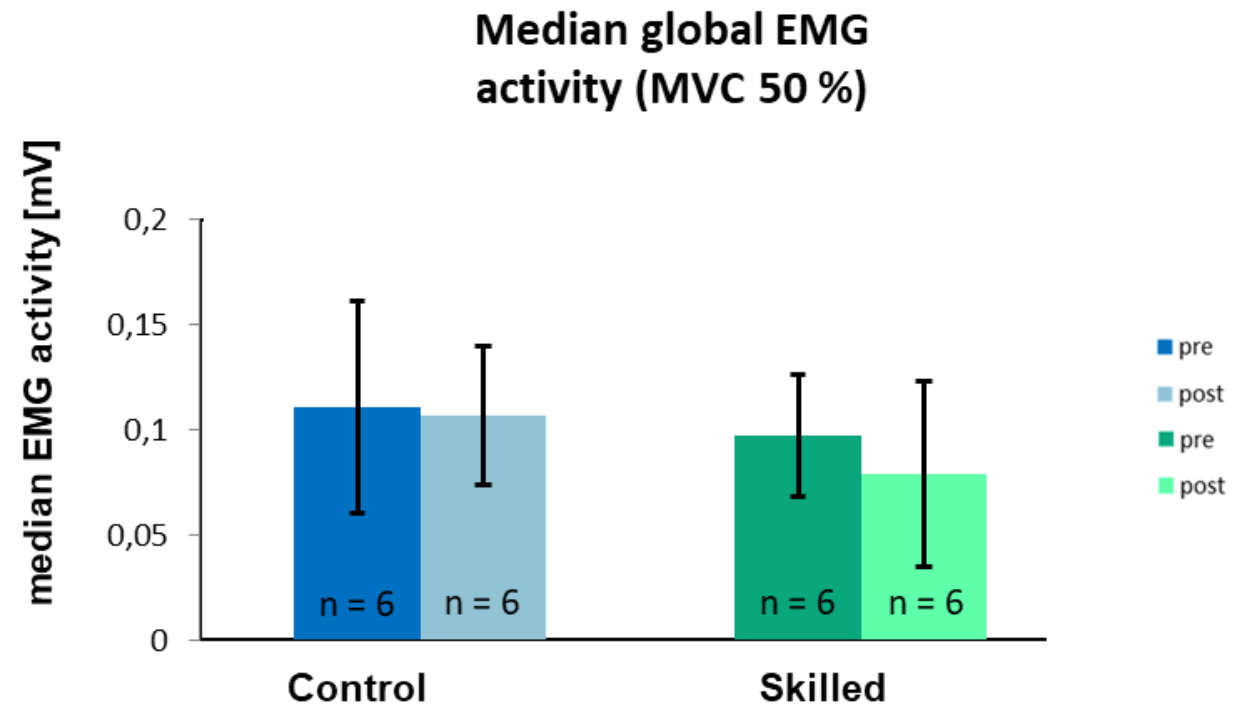

Figure 48: Median global EMG activity [mV] for pre- and post-test at $50 \% \mathrm{MVC}[\mathrm{mV}]$ for control and skilled in SOL. 
Table 9: Global EMG activity [mV] for pre- and post-test at $50 \% \mathrm{MVC}$ for control and skilled in SOL:

\begin{tabular}{|c|c|c|c|c|}
\hline Group & $\begin{array}{c}\text { Mean EMG Activity } \\
{[\mathrm{mV}]} \\
\text { pre }\end{array}$ & $\begin{array}{c}\text { Mean EMG Activity } \\
{[\mathrm{mV}]} \\
\text { post }\end{array}$ & $\begin{array}{c}\text { Median } \\
\text { EMD } \pm \text { STDEV } \\
{[\mathrm{ms}]} \\
\text { pre }\end{array}$ & $\begin{array}{c}\text { Median } \\
\text { EMD } \pm \text { STDEV } \\
{[\mathrm{ms}]}\end{array}$ \\
\hline Control & 0.115 & 0.127 & $0.111 \pm 0.033$ & $0.107 \pm 0.051$ \\
\hline Skilled & 0.097 & 0.073 & $0.097 \pm 0.044$ & $0.079 \pm 0.029$ \\
\hline
\end{tabular}

The median global EMG activity in the control group was measured as $\mathrm{gEMG}_{\text {pre }}=0.111 \pm 0.033 \mathrm{mV}$ and $\mathrm{gEMG}_{\text {post }}=0.107 \pm 0.051 \mathrm{mV}$; skilled performed with $\mathrm{gEMG}_{\text {pre }}=0.097 \pm 0.044 \mathrm{mV}$ and $\mathrm{gEMG}_{\text {post }}=0.079 \pm 0.029 \mathrm{mV}$ (Table 9 and Figure 48).

\subsubsection{Author contributions}

Robin Diedrichs and Beate Pröttel conceived and designed experiment and research with support of Utku S. Yavuz. Experiments were performed by Robin Diedrichs and Beate Pröttel. Robin Diedrichs analyzed data. Those data were interpreted by Robin Diedrichs. Figures were prepared by Robin Diedrichs. The manuscript was created by Robin Diedrichs. 


\section{Discussion}

By planning and conducting the experiments presented in this thesis, we addressed the question of whether differences in movement performance of skilled bodily motor tasks, visible from the outside, are caused by neural adaptations on spinal cord level due to long-term motor skill training. Muscle's interplay is complex and still not fully understood, especially on the level of motor units. Our approach of non-invasive investigation of myoelectric signals via HDsEMG, to access the motoneuron pool instead of single MUs, allows us to investigate and examine neuromuscular control (Yavuz et al., 2015, 2018). This comes along with the sequent task of processing MU-behavior from HDsEMG recordings with a reliable tool (Holobar et al., 2007 b; Yavuz et al., 2015; Negro et al., 2016).

We found significant differences in distribution of reciprocal inhibitory reflex amplitudes, between lower limb's extensor and flexor muscle (study 1). Furthermore, we found that neural drive, i.e. reflex amplitudes, of lower limb's flexor muscle SOL and GM was significantly different in a group of skilled martial artists compared to an unskilled control group (study 2). With a similar approach we studied and found insignificant differences, but trends, in neural drive and functional aspects of lower limb's muscles between a group of skilled Yoga practitioners and an unskilled control group (study 3).

\subsection{Differences in neural drive of lower limb's muscles - Study 1}

In this chapter we discuss the differences in neuromuscular control of extensor and flexor muscles of the lower limb. Triceps surae muscles (GM and SOL) and TA are the main muscles contributing to posture as well as to the gait cycle by controlling ankle flexion and extension; GM also supports knee extension. To provide muscles' interplay during motor tasks, reciprocal inhibition supports controlling this functional interplay. Previous studies focused on modulation of reciprocal inhibition during gait in muscle of cat (Pratt et al., 1987) and in human (Capaday et al., 1990; Lavoie et al., 1997; Petersen et al., 1999). We found this interplay is most likely based on differential reciprocal inhibition. Our results support this hypothesis strongly since we measured a significant larger median value for amplitudes of the reciprocal inhibition of TA motor unit's amplitudes. TA MUs showed a 4-fold larger reflex amplitude range during reciprocal inhibition compared to MUs of GM and SOL (Figure 32). It should also be taken into account that TA is the only muscle contributing to 
dorsal extension and that it traverses a remarkable distance. This makes TA a unique muscle with a very diverse composition of type I and type II muscle fibers along its length (Lexell et al., 1994).

Study 1: It was shown on a large population of motor units, that TS muscle group inhibits TA reflexes strongly (Figure 32), but that this is not true vice versa (Yavuz et al., 2018). Our results are referring to the accepted model of agonist and antagonist muscle interplay for lower limb muscles (Nielsen et al., 1993 a) and it is in line with former experiments (Crone et al., 1987). Petersen et al. (Petersen et al., 1999) investigated changes in the transmission of the $I_{A}$ inhibitory pathway in the walking-cycle, more specific in swing and stance phase. It is very likely that $I_{A}$ input is non-uniformly distributed. Referring to Figure $\mathbf{8}$ and Figure $\mathbf{1 7}$ we can expect at least one interneuron in the investigated reciprocal inhibitory pathway between TA and TS muscle group. Nielsen and Kagamiharat (Nielsen et al., 1992) investigated the disynaptic reciprocal inhibition of lower limb muscles during cocontraction and found it to be depressed for both antagonistic motor unit groups (Yavuz et al., 2018). By designing our experimental setup, we have chosen an approach, focusing on sustained isometric contraction, to exclude modulatory effects (Nielsen, 2016) of other ascending and descending pathways.

With regard to Henneman's size principle and assuming a uniform reciprocal inhibition, early recruited MNs are smaller in diameter and therefore excited earlier, presenting higher reflex amplitudes (Henneman et al., 1965; Yavuz et al., 2018). We found that TA has a negative correlation between reflex amplitude and recruitment threshold (Figure 31) (Yavuz et al., 2018). At low contraction levels, as represented by 10 and $20 \%$ MVC, mainly slow twitch motor units are activated (Kandel et al., 2012; Yavuz et al., 2018). Innervation of fatigue-resistant muscle fibers provides possibility to compare reflex responses of MUs from TA and TS muscle group. Only MUs with a similar background discharge rate were used for further calculation (Yavuz et al., 2018). This confirms our hypothesis for a differential transmission of reciprocal inhibitory input to MUs from TA and TS muscle group (Yavuz et al., 2018).

Functionally, the diverse reciprocal inhibition between lower limbs extensor and flexor muscles supports the different tasks for both. SOL is mainly tonically active in quiet standing, whereas GM shows bursts of activations (Yavuz et al., 2018). The human body acts like an inverted pendulum, in which SOL stabilizes human posture during micro-falls (Loram et al., 2011). Also modifiability of input by $\gamma$-MNs during the task has probably a great impact on the observed differential distribution; fusimotor drive from muscle spindles' active endings is higher, when movement is prevented (Pierrot-Deseilligny et al., 2012). Furthermore, fusimotor drive seems to contribute to moment-tomoment control of movement (Aniss et al., 1990; Pierrot-Deseilligny et al., 2012) and should 
Discussion

therefore play an important role in sustained isometric contraction. Further studies supporting HDsEMG recordings should be conducted at different points of the entire length of TA, to investigate motor unit behavior of this heterogeneous muscle. Besides the result of differential distribution of reciprocal inhibition between lower limb's extensor and flexor muscles in a heterogeneous group of individuals, it is of great interest if adaptations in the neuromuscular system lead to differences in monosynaptic excitatory H-reflex pathway; this can be investigated in groups with long-term motor training experiences and control groups (Nielsen et al., 1993 b; Adkins et al., 2006).

\subsection{Effects of long-term motor skill training - Study 2 and Study 3}

In this chapter we discuss how neural drive changes with regard to long-term motor skill training. Neural drive differs between groups of regularly trained subjects and control (Nielsen et al., $1993 \mathrm{~b}$; Adkins et al., 2006; Ogawa et al., 2009). This difference is thought to arise, for example, from longterm motor skill training. As previously mentioned, power and endurance training affect neural plasticity as well (Adkins et al., 2006). Performing one type of training comes along with the other two types of training, automatically. To study the evidence of neural plasticity in human directly on spinal cord level, the approach would be to open the spinal cord surgically and investigate innervations directly, take probes and further analyze them under physiological conditions. Since this approach is precluded for human, researchers investigated in cat (Burke et al., 1974; Hultborn et al., 1979), mouse (Almeida-Silveira et al., 1996; Chen et al., 2001) and monkey (Wolpaw et al., 1989; Carp et al., 1994, 1995) the underlying neuromuscular properties that are assumed to be responsible for the execution and planning of movements. For human experiments the approach of sEMG became popular (Adkins et al., 2006; Knikou, 2008; Pierrot-Deseilligny et al., 2012).

Study 2: We examined differences in H-reflex amplitudes between groups of long-term skill trained martial artists and a control group. We found significant differences $(p<0.05)$ in $\mathrm{H}$-reflex amplitudes between skilled and control for SOL and GM at $10 \%$ MVC (Figure 36), respectively $20 \%$ MVC (Figure 37). These differences are thought to arise from different afferent muscle spindle input (Nielsen et al., 1993 b; Adkins et al., 2006) and result in different neural drive of $\alpha$-motoneurons with regard to long-term motor skill training. It should also be mentioned that co-contraction training leads to higher fusimotor drive (Pierrot-Deseilligny et al., 2012), which in turn affects excitability of $\alpha$-MNs.

RMS of EMG reflects physiological activity of motor units during contraction (Fukuda et al., 2010). No significant differences are found in RMS at $20 \%$ MVC between groups for GM (Figure 34) and SOL 
Discussion

(Figure 35). This indicates that overall electrical muscle activity does not differ between groups, leading to the assumption that, in total, a similar count of muscle fibers is activated in both groups.

We further examined recruitment thresholds for control and skilled group at both contraction levels. We illustrate this by early and lately recruited MUs for SOL at $10 \%$ MVC (Figure 38) and $20 \%$ MVC (Figure 39) and as scatter plot for SOL at $10 \%$ MVC (Figure 40) and $20 \%$ MVC (Figure 41). Reflex amplitudes, with regard to recruitment threshold for early (first $5 \%$ while exerting the double amount of MVC while performing ramp contraction) and lately recruited MUs (last $10 \%$ while exerting the double amount of MVC while performing ramp contraction), show no significant difference. As a trend, we consider higher reflex amplitudes in lately recruited MUs come along with increased amount of exerted force in-between groups (Adkins et al., 2006). Skilled subjects trended to perform at both contraction levels with slightly higher recruitment thresholds. This could indicate higher drive to and earlier activation of $\alpha-M N s$ with a larger diameter in the skilled group (Henneman et al., 1965; Yavuz et al., 2018). It would be likely that this difference is a result of neuromuscular adaptations, taken place by long-term motor skill training (Adkins et al., 2006). We cannot say if the amount of activated $\alpha-M N s$ with a larger diameter is higher in the skilled group due to a higher count of fibers of this type or by interconnectivity of neural inhibitory or excitatory pathways; further analysis and recruitment of more subjects would be needed to statistically strengthen our results.

Study 3: We used a similar protocol in pre- and post-test to investigate reflex amplitudes in $\mathrm{H}$-reflex in groups of long-term skilled trained Yoga practitioners and control before and after stretching intervention. We have chosen this approach to analyze neural drive within the motoneuron pool with regards to the effect of a stretching intervention, to analyze differences in neural drive due to long-term motor skill training in a group of Yoga practitioners and a moderately active control group.

No complete H-reflex/M-wave tuning curve (Figure 18) was measured for each subject. We needed to apply high stimulation pulses which mostly evoked direct motor responses instead of H-reflexes. Since electricity propagates from anode to cathode, the tissue in between must be passed, leading to stimulation of nerve fibers in a size-dependent order. Subjects in study 3 performed H-reflex measurements in prone position, whereas in study 1 and 2 subjects were seated. Prone position could be a reason for excitation of efferent fibers causing $M$-waves during stimulation. The knee is slightly more bent, nearly locked $\left(170^{\circ}-180^{\circ}\right.$ knee angle), compared to the sitting position $\left(120^{\circ}\right.$ knee angle) in the other performed experiments (Yavuz et al., 2018). This could have led to a situation where afferents could not be accessed by electrical stimuli. Furthermore, electrical resistance between anode and cathode changes due to the underlying nerve and surrounding tissue; 
the tissue is pushed against the popliteal fossa, which could have led to higher excitation values and resulted in excitation of efferent fibers. Due to differences in tissue organization and muscle ratio between genders, the accessibility of TN in the popliteal fossa could have led to this outcome. Study 1 and 2 mostly investigated male subjects. Yavuz et al. (Yavuz et al., 2010) dealt only with supramaximal stimuli to investigate differences in EMD between genders. In turn, we estimated the direct motor response of the EMD before and after the intervention. We found no significant differences $(p>0.10)$ for EMD calculations for skilled and control group regarding pre- and post-test (Figure 47), but a trend. We can assume the found difference of a $23 \%$ shorter EMD for the control group in pre-test and $30 \%$ shorter in post-test. Since EMD also increases with age and fatigue (Yavuz et al., 2010), both depending on changes in neuromuscular systems due to aging, we assume those differences arose from neuromuscular adaptations due to long-term stretching-training in Yoga. It is not clear, which structures or neural components support the shortened EMD in the skilled group. One approach is to support the findings with an examination of the effect of stretching training combined with muscle biopsy to analyze differences in muscle properties. Reflex sensitivity is for example present even after prolonged, passive stretch (Avela et al., 1999 a) and reflex sensitivity is reduced even days after stretch-shortening cycle (Avela et al., 1999 b). This supports our hypothesis that both intrinsic changes, as well as altered excitability of neural structures involved in reflex arcs, take place due to stretching training and are consolidated by long-term training. Further examination of more subjects should be conducted.

Force output in both groups is smaller after intervention (Figure 44), which is in line with a former study by Matsuo et al. (Matsuo et al., 2013). This can be interpreted by the following: the overlap of actin and myosin filaments is at optimum while at rest, but not reestablished directly after the stretch (Schmidt et al., 2007). We hypothesize that higher variance in skilled compared to control in post-test could arise from long-term Yoga stretching-training, resulting in altered structural adaptations on muscle fiber and sarcomere level.

Variance of MUDR RMS is lower in pre-test compared to post-test in both groups, but in general lower in skilled (Figure 45); these results are statistically not powerful enough to support any hypothesis. Calculating the RMS of discharge rate of all firing MUs ( $n=1037)$ provides neither significant results, nor do they show a clear trend. This displays the necessity to plan and execute further experiments and to evaluate clear conditions to exclude side effects and to study MU discharge behavior.

The increase in stretching angle of $5.2 \% \pm 2.9 \%$ in control and of $11.2 \pm 5.4 \%$ in skilled (Figure 46 ) is in line with a former experiment by Matsuo et al. (Matsuo et al., 2013). We hypothesize that this is 
Discussion

explained by short-term effects of stretch on tendons and ligaments, whereas the difference of 2fold higher increase in percentage of hip angle in skilled, regarding pre- and post-test, compared to control, could be explained by long-term adaptations of the underlying (neuro-)muscular properties.

\subsection{Subjects: influence of physical activity and training}

Neural drive is also influenced by normal day-life activities like bicycling, walking, jogging etc. Since training to walk backwards has an influence on reflex amplitudes during H-reflex of SOL (Capaday et al., 1990), we hypothesize that bicycling has a great impact on the fine interplay of TS muscles and TA (Pyndt et al., 2003). Pedaling seems to alter the interplay between extensor and flexor muscles in a complex way, leading to fine muscle interplay, and therefore resulting in neuromuscular adaptations. It must be taken into account that daily bicycling or walking to work and back for example for 20 minutes will have a great impact on neural plasticity and neuromuscular properties (Adkins et al., 2006; Pierrot-Deseilligny et al., 2012).

We can examine and display differences in neuromuscular control of a subject, achieved by training, for a long period of time. Training at least two times a week, for a period of at least three years, results in an significantly altered control of the lower limb muscles GM and SOL compared to control group (study 2). This is thought to be a result of altered motor control on a neural level, since the underlying reflex arc of $\mathrm{H}$-reflex is monosynaptic and represents involuntary control of the homonymous muscle (Hoffmann, 1910; Liddell et al., 1924). Training of specific motor tasks like postures and stances in a conscious and aware way (Feldenkrais, 1972; Hatsumi et al., 2008; Arus, 2013), seems to have great impact on neuromuscular control and on internal communication and integration of afferent information as well as on altered neural drive to extensor and flexor muscles. It results in various improvements like pain reduction in movement, improvements in associated diseases and better functionality with regard to injury or chronic diseases (Feldenkrais, 1972). The resulting effects of training are present on spinal cord level, as well as in motor cortex even after a short period of time and persist for a certain period of time, depending on the amount and time of training experience (Adkins et al., 2006). Balance training seems to alter even higher neural networks and evokes corticospinal adaptations (Schubert et al., 2008).

Both groups were asked to answer a verbal survey about their daily activity level, since we concluded that the short form of International Physical Activity Questionnaire (Fogelholm et al., 2006) does not give insight into our leading question. It should be discussed that the group of long- 
term trained martial artists is, due to their training, more physically active than the control group. It can be assumed that both the groups of long-term trained martial artists as well as the group of long-term trained Yoga practitioners are more athletic in general. We know that endurance and strength training change the connectivity of neural networks and reflex arcs (Adkins et al., 2006; Vila-Cha et al., 2012) with the result that power trained subjects show decreased H-reflex excitability and increased V-wave compared to strength and endurance trained athletes. This suggests, that power training is affecting less activated motoneurons, excited by the $I_{A}$ afferent volley, compared to control group (Adkins et al., 2006); it has also been shown that several weeks of strength training result in increased H-reflex and V-wave amplitudes (Adkins et al., 2006).

The impact of endurance training on spinal plasticity is thought to change not only one, but several neural circuitries (Adkins et al., 2006). SOL H-reflex amplitude is decreased in ballet dancers, but on the other hand H-reflex amplitudes are higher in extremely active people compared to moderate and sedentary subjects (Nielsen et al., 1993 b). Furthermore endurance trained athletes show higher excitability of reflex arcs for patellar and H. Achilles tendon (Koceja et al., 2004). Experienced swimmers for example show enhanced reflex excitability of SOL compared to a non-trained control group (Ogawa et al., 2009). Targeting $M$. infraspinatus, it has been shown that the underlying neural reflex circuitry is altered due to glenohumeral instability of the shoulder comparing normal and athletic shoulders (Augé et al., 2000).

It turns out that endurance training has no clear outcome, whether an increase or decrease of excitability of reflex arcs on spinal level occurs. For that reason, an in- or decrease in reflex excitability and $\mathrm{H}$-reflex elicitation seems to be extremely task-dependent (Adkins et al., 2006; Thompson et al., 2009; Pierrot-Deseilligny et al., 2012). The result is effective and fine muscle interplay, resulting from the desired tasks. This is the reason for different results investigating even the same muscle in different trained subjects in all named investigations. In animal experiments it had been shown, that endurance training even affects organization on motor cortex level, resulting in angiogenesis (Kleim et al., 2002; Swain et al., 2003).

In general, it can be concluded that skill training automatically involves endurance and power training, since none of these aspects of training can be performed without the other. If the focus is more on skill training, the effect on the results of strength and endurance training is less; if endurance training is performed the impact on strength and skill is less; if subjects perform strength training the resulting effect on skill and endurance is less. We can also assume that the effect of training is a holistic one. To achieve optimization over-all, a conscious way of training with an holistic approach, as stated and provided by Feldenkrais (c.f. chapter 5.3), should be trained (Feldenkrais, 
Discussion

1972; Hatsumi et al., 2008; Arus, 2013). Since training of stances and postures in erect position has a great impact on the stabilizing muscles of ankle and knee, it is reasonable that the group of skilled martial artists perform with higher reflex amplitudes compared to non-trained control group.

We assume that neuromuscular adaptations have taken place in the group of trained martial artists and in the group of trained Yoga practitioners. The assessed muscles in martial arts training massively involve activation of lower limb muscles for a stable stance and lower back muscles for proper alignment of hip and thigh. In Yoga, mainly the posterior chain is targeted by leg-stretching techniques, resulting in neuromuscular adaptations.

Those adaptations can occur in afferent $\left(I_{A}, I_{B}\right.$ and II), efferent ( $\alpha$ - and $\gamma$-motoneurons) motoneurons, in muscle fibers (type $I_{A}, I_{B}, I_{I}$ and $I$ ) and intrinsic muscle properties can change as well (PierrotDeseilligny et al., 2012). On the next higher levels, training experience extremely influences the interplay of muscles for smooth and fine movements. Sequent motor tasks are only accomplished with loading and executing motor programs, which themselves are built up by motor modules. Cerebellum integrates afferent sensory information of spinal cord. Midbrain has a directing function of those clues; basal ganglia seem to integrate information and contribute to voluntary movement; motor cortex (primary- and pre-motor cortex, SMA) is responsible for execution, preparation and coordination of motor tasks (Kandel et al., 2012).

As we see, modifiability can and does occur at every of these stages, giving the organism the possibility to adapt its motor behavior according to its environment. Holistic investigations on the underlying neural mechanisms seem crucial by technical limitations. Motor experiences depend on the complex interplay of spinal circuities under the influence and modulation of several higher centers (forebrain, cerebellum etc.). Therefore, several conditions influenced (our) data acquisition. This must be taken in mind to properly interpret these data.

\subsection{Technical considerations}

\subsection{3 iEMG, sEMG and HDsEMG}

The main advantage of iEMG is that the measured spiking units are the ones that are grasped, i.e. 'hooked', with the electrode's tip. By hooking several fibers, it is getting more difficult to extract spiking events from a single motoneuron. On the other hand, only one hooked fiber gives an extremely precise and reliable picture of the firing event (Milnik, 2012). sEMG shows big advantages 
for investigations of not only single muscle fibers but the entire muscle (Adkins et al., 2006). Not only a few MUs are measured but the overall activity of the entire signal spreading over the muscle bulb in a non-invasive way. But it must be mentioned that iEMG records internal muscle fibers, whereas sEMG represents the discharges of fibers located on, respectively near, the outer muscle bulb. Since muscle fibers located in the muscle are less oxidative and mostly fast-glycolytic and fibers near the bulb are more dependent on oxygen (fast- and slow-oxidative) (Henriksson-Larsén et al., 1983). Also rate of fatigue, muscle fibers diameter, MN diameter of the innervating axons are different between muscle fibers (Kandel et al., 2012). This leads to the conjecture that results of studies, investigating with different EMG approaches and techniques, are not directly comparable along each other.

HDsEMG comes to solve the problem of single identified MUs in iEMG recordings with decomposition of the several firing events into single motor units (Holobar et al., $2007 \mathrm{~b}$; Farina et al., 2010; Yavuz et al., 2015). It should be mentioned as well, that this non-invasive approach leads to less discomfort and pain compared to needle insertions used in IEMG recordings. Discomfort causes change in the downward flow from CNS and alters excitability of spinal neurons (Kandel et al., 2012).

\subsubsection{Force transducer}

The force transducer we used was a built-in device of the Biodex System $3 \mathrm{Pro}^{\circledR}$. Since torque was measured with a potentiometer as an electrical potential difference [V] for standardization of force amounts, it is very difficult to transform these amounts of volts into an according amount of force [N] accurately. Mean EMG discharge activity defines in a precise way MVC amounts to adjust percentages of the maximum amount during experiments (Farina et al., 2017). 
Outlook

\section{Outlook}

Motor behavior and learning due to motor experiences is present in every animal. Every kind of learning is directly associated with motor behavior or with its support. It is needed for locomotion itself but nevertheless for learning a skill, trade or speech and is even present in dreaming.

We accept that motor learning is important for the organism itself to interact with the environment and for interaction with and in society. These are only a few examples that demonstrate the great importance and necessity of researching motor control to understand the interplay and modifiability of neural compounds; learning, consolidation, modifying and reconsolidation build the basis for every kind of motor learning (Schiller et al., 2011; Schwabe et al., 2014). From an evolutionary point of view the research of motor learning is of great interest since organisms are only able to develop due to interaction with the environment and by social interaction. This interaction in everyday life requires fast adaptations in particular tasks to accomplish tasks and react on changes in the environment. A disturbance of motor behavior can be present due to internal developmental events as Parkinson's or Alzheimer's disease (Kandel et al., 2012; Pierrot-Deseilligny et al., 2012), or due to external events that cause persisting damage of functional components of movement and locomotion or due to neural damage resulting in altered proprioceptive control, or due to altered processing or integration of information caused by disease (Pierrot-Deseilligny et al., 2012). Finding treatment for those types of dysfunctions is the field of rehabilitation and neurorehabilitation.

Physical rehabilitation can be accomplished in several ways and is offered in vast ways. The question for a helpful treatment for example of common everyday life issues in our actual society like lower back pain or cervical pain is quite old (Murray, 1939; Magnuson, 1944) and still of great importance (Williams, 2009; Dideriksen et al., 2014; Vaisy et al., 2015). Physiotherapists offer guidance of exercises and therapy itself for different types of functional misbehaviors. Also, the aware performance of exercises is extremely important, and it supports healing and reduction of pain and contributes to refinement of movements and thereby enhances quality of life (Feldenkrais, 1972). One way thought to achieve this, is the performance of Yoga exercises, which became very popular in the past decades and is accessed by a broad group of people. In the concept of Iyengar Yoga a posture is most often achieved with the co-contraction of extensor and flexor muscle (Williams, 2009; Ni et al., 2014).

Several studies wanted to point out the results of yoga training with respect to physical and mental structures (Williams, 2009; Cox et al., 2010; Tilbrook et al., 2011; Michalsen et al., 2012; Cramer et al., 2013; Myers, 2013; Pröttel, 2017). On the one hand, the effects of long-term motor skill training 
Outlook

are various; on the other hand the origin of, for instance, lower back pain or mental diverge from the norm remains unclear. Nevertheless, lyengar yoga is thought as a treatment for spinal pain (Eisenberg et al., 1998) and is considered as therapeutical, quasi 'medical' yoga (Crow et al., 2015). Mid-term training of 16 (Williams et al., 2005) and 24 weeks (Williams et al., 2009) provides significant reduction in lower back pain, function disability, and pain medication usage. Cox et al. showed in a study a reduction in lower back pain after 4 weeks of training only (Cox et al., 2010). Chronic neck pain is reduced by lyengar yoga after 10 weeks of training in rest and in motion (Michalsen et al., 2012). Besides reduction of chronic neck pain, lyengar yoga practitioners report less disability and better mental quality of life (Cramer et al., 2013).

For the protocol, a posture called Supta-Pādāngushthāsana was performed where the name arrives from supta from "Iying down", pādā from "foot" and āngushta from "big toe" performed in āsana Yoga. Performance of this exercise is thought to improve mobility of hip joints, to better sciatic pain and to reduce the appearance of paralysis of the lower limb (Iyengar et al., 2013). This posture is experimental relatively easy to perform for the subjects and it provides stretching of all muscles of our interest: $\mathrm{GM}, \mathrm{SOL}, M$. peroneus and $M$. semitendinosus.

\subsection{Feldenkrais}

Aiming for a holistic approach, the Feldenkrais method should be named. Feldenkrais shaped a physical-oriented exercise therapy based on kinaesthesia and proprioception (Feldenkrais, 1972). Kinaesthesia describes the sensation of motion and the possibility to control body parts unconscious. Proprioception gives the performer mainly information about sense of position, sense of force and sense of motion. This sensing is based on accessed information by inner organs (Feldenkrais, 1972). This method is thought to improve bodily functions, awareness and health (Hillier et al., 2015). It is also used in relaxation procedures and to reduce anxiety (Kolt et al., 2000), and to support stretching by more muscle length in hamstring muscles (Stephens et al., 2006); it shows significant improvement in dexterity of the dominant hand due to sensory awareness lessons (Bitter et al., 2011), as well as significantly improved balance and gait speed (Vrantsidis et al., 2009). The resulting positive physical and mental differences after a period of training are quite diverse (Ruth et al., 1992; Laumer et al., 1997; Johnson et al., 1999; Grübel et al., 2003; Quintero et al., 2009; Hillier et al., 2010).

The Feldenkrais method was developed to reduce pain, and to provide practitioners finer movement quality and improved wellbeing (Feldenkrais, 1972). It is supposed, that reorganization and 
Outlook

refinement of cortical and subcortical networks involved in motor control are responsible for the effect of Feldenkrais training by reorganization of connections from the motor cortex to the body. This theory takes into account, that learning processes, from baby to infant, still endure in children, youngsters, adults, middle-aged and elderly persons (Feldenkrais, 1972) and neural plasticity is not exclusively bound to the early stages of development. This approach is of extremely high importance, since this kind of internal communication and refinement of circuitries on even higher neural levels is achieved in many holistic approaches of movement training. When it comes up to hard restrictions due to malformation of limbs or amputated limbs support, orthosis and prosthesis are a way to re-achieve control of the $\operatorname{limb}$ and therefore the entire body including a re-gain of personal freedom. This treatment is often supported with Feldenkrais training.

\subsection{Further experiments - Consolidation and Reconsolidation}

The conducted experiments in this thesis build the bottom line for further investigations. We planned further studies investigating the behavior and neuromuscular control while standing and performing stances, respectively postures, in detail. It is planned to apply HDsEMG electrodes to lower limb muscles GM, SOL, and spine erector M. erector spinae to examine EMG activity and $\mathrm{H}$ reflex amplitudes while participants are standing and perform a martial arts stance. Since motor learning is crucial for adaptation in an environment, it is of valuable significance and relevance to research learning in general.

We know that learning and acquisition of new motor tasks takes place in a phase called consolidation (Walker et al., 2003). After execution, a successfully learned task can still be altered; this progress is called reconsolidation (Walker et al., 2003). Reconsolidation allows the organism to modify present motor behavior (Schiller et al., 2011; Wymbs et al., 2016) to achieve customization for specific tasks or to react to certain dysfunctions during lifetime. We also know that reconsolidation relies on molecular mechanisms (Etcheberrigaray et al., 2007) and we know about its clinical relevance (Schwabe et al., 2014). We planned a further experiment with a group of longterm skilled martial artists and a control group. The subjects will stand on two adjustable force plates. Subjects will be slightly deflected while performing the stance; differences in support of stabilizing motor control shall be monitored with regard to neuromuscular control. HDsEMG signals will be recorded for SOL, GM and $M$. erector spinae to investigate those expected differences between both groups before a period of reconsolidation. A second test will be performed the next day after a period of sleep. We expect the reconsolidation occurs in this period of sleep (Walker et al., 2003; Schiller et al., 2011). On the second day, subjects will be deflected in the same way as on 
Outlook

the day before and HDsEMG signals will again be recorded for SOL, GM and $M$. erector spinae. With this approach we could be able to (i) monitor non-skilled group's acquisition of a new posture and (ii) study the neuromuscular learning process over night during consolidation. On the other hand, the group of skilled martial artists provides us information about reconsolidation (iii). The stance is already part of the movement repertoire of the skilled group but by disturbance movement representations can be altered and re-wired, since reconsolidation occurs (Wymbs et al., 2016). 


\section{Summary}

By approaching the topics of lower limbs' extensor and flexor muscle's underlying neural circuitries and neural plasticity on spinal level with HDsEMG recordings, also in relation to long-term motor skill training, we found significant differences in motor unit behavior and neuromuscular control.

Study 1 showed significant differences in reciprocal inhibition of lower limb's extensor (TS) and flexor (TA) muscles. We found median reciprocal inhibitory reflex amplitudes for TS muscles GM and SOL to be 4-times smaller compared to TA. The result points out that these reciprocal inhibitory reflex amplitudes of lower limb's extensor and flexor muscle are not symmetrically distributed, but differentially. TS muscle group inhibits TA reflexes strongly, but this is not true vice versa.

In study 2 we examined the effect of long-term motor skill training on neural plasticity in terms of $\mathrm{H}$ reflex, reflecting neural plasticity on spinal level. We found median excitatory reflex amplitudes in lower limb's extensor muscles to be significantly higher in long-term motor skilled subjects compared to moderately active control.

Study 3 showed insignificant results due to setup restrictions and subject number. As a trend, we found long-term motor skill training shortens EMD of lower limbs extensor muscles, as the result of long-term stretching training. In functional terms we found stretching training to improve mobility and range of motion. 
Figures and Tables

\section{Figures \& Tables}

FIGURE 1: DEPICTS DORSAL VIEW OF REGIO CRURIS POSTERRIOR AND FOSSA POPLITEA RIGHT SIDE. FASCIA CRURIS REMOVED AND M. GASTROCNEMIUS DISSECTED (BY SOBOTTA ET AL., 2017, MODIFIED.)

FIGURE 2: DEPICTS DORSAL VIEW OF REGIO CRURIS POSTERRIOR AND FOSSA POPLITEA RIGHT SIDE. M. GASTROCNEMIUS AND M. SOLEUS DISSECTED (BY SOBOTTA ET AL., 2017, MODIFIED).

Figure 3: DEPICTS VENTRAL VIEW OF REGIO CRURIS ANTERIOR, RIGHT SIDE, CONTAINING M. TIBIALIS ANTERIOR (BY SOBOTTA ET AL., 2017, MODIFIED).

FIGURE 4: EXPLANATION OF THE LEVELS OF MOTOR CONTROL (FOREBRAIN, BRAIN STEM AND SPINAL CORD). GREEN ARROWS DEPICT DIRECT ACTIVATION BY MOTOR AREAS OR INDIRECT BY BRAIN STEM'S DESCENDING SYSTEMS ISSUING INTO SPINAL CORD. AFFERENT INPUT IS RECEIVED AT ALL LEVELS. ORANGE ARROWS SHOW INFLUENCE OF CEREBELLUM AND BASAL GANGLIA, GREEN ARROWS SHOW DIRECT MOTOR COMMANDS BY MOTOR AREAS ONTO SPINAL INTERNEURONS AND MOTONEURONS., BLUE ARROWS INDICATE INTEGRATION OF INFORMATION IN BASAL GANGLIA (BY KANDEL ET AL., 2012, MODIFIED). 16

Figure 5: Popliteal fossa depicting Stimulation AREA Of THE PERIPHERAL NERVE (BY SCHMIDT ET AL., 2007, MODIFIED)......17

Figure 6: MYELINATION OF AN AXON BY SCHWANN CELLS (INTERNODE DISTANCE IS ABOUT 1 MM), EACH SHEATH IS FORMED BY AN INDIVIDUAL SCHWANN CELL (BY KANDEL, 2012, MODIFIED) ........................................................................ 18

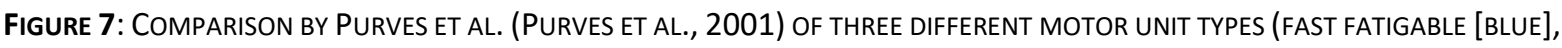
FAST FATIGUE-RESISTANT [ORANGE] AND SLOW [PURPLE]). SINGLE MOTOR UNIT RESPONSES ARE SHOWN. THE X-AXIS SHOWS INDIVIDUAL TIME COURSE, Y-AXIS REPRESENTS FORCE DEVELOPMENT (A, B) AND PERCENTAGE OF FORCE (C). (A) ACCORDING TO SINGLE MOTOR UNIT ACTION POTENTIAL MUSCLE TENSION CHANGES. (B) MUSCLE TENSION IN RESPECT OF REPETITIVE SUBSEQUENT STIMULATION. (C) TETANIC STIMULATION EVOKES MAXIMUM TENSION (BURKE ET AL., 1974, MODIFIED). ...... 20

FIGURE 8: NEURAL CIRCUITRY OF HOMONYMOUS MUSCLE LEFT: CROSS-SECTION OF SPINAL CORD; MIDDLE: AFFERENT AND EFFERENT INNERVATION OF M. SOLEUS; RIGHT: LOWER LIMB MUSCLES (BY SCHMIDT ET AL., 2007, MODIFIED).

Figure 9: THE FIGURE SHOWS GENERATION OF APS BY CHANGES IN MEMBRANE-CONDUCTANCE FOR SODIUM AND POTASSIUM, RESULTING DEPOLARIZATION AND REPOLARIZATION PHASE; ACHIEVED BY PATCH CLAMP TECHNIQUE BY HODGKIN AND HUXLEY, 1952 (FIGURE BY KANDEL ET AL., 2012, MODIFIED).THE DEPOLARIZATION OF THE MEMBRANES POTENTIAL IS FOLLOWED BY AN AVALANCHE-LIKE OPENING OF SODIUM CHANNELS WHICH IN TURN LEADS TO OPENING OF POTASSIUM CHANNELS, FOLLOWED BY REPOLARIZATION ACCORDING TO EQUILIBRIUM POTENTIALS OF THE INVOLVED IONS......

FIGURE 10: FOR A SHORT PERIOD OF TIME DEPOLARIZATIONS OF THE AXON CANNOT ELICIT A NEW AP. AFTER 2.5 MS DEPOLARIZATIONS FAR ABOVE THRESHOLD CAN ELICIT A NEW SMALLER AP, WHEREAS AFTER $\sim 4.5$ MS AN AP WITH SIMILAR SIZE LIKE THE FIRST DEVELOPED AP CAN BE GENERATED (BY SCHMIDT ET AL., 2007, MODIFIED).

FIGURE 11: CROSS SECTION OF NEUROMUSCULAR JUNCTION, T: AXON TERMINALS, M: MUSCLE FIBER, ARROW: JUNCTIONS WITH BASAL LAMINA SCALE: $0.3 \mu \mathrm{M}$ (BY WIKIMEDIA COMMONS, ORIGINALLY UPLOADED TO EN.WIKIPEDIA ON $10^{\text {TH }}$ MARCH 2006; originally SOURCE: Synapse Web at the National Institute of Mental Health, National InStitutes of Health,

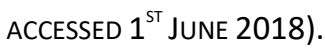

FIGURE 12: ELICITED SYNAPTIC CURRENT CAUSED BY TRANSMITTER RELEASE OF MOTOR NEURON TERMINALS DECAYS ALONG THE MUSCLE FIBER. CURRENT PROPAGATES IN THE INTERIOR OF THE MUSCLE FIBER AND SINCE THE ELECTRIC CIRCUIT MUST BE CLOSED, IT FLOWS BACK IN THE EXTERIOR OF THE MUSCLE FIBER (BY KANDEL ET AL., 2012, MODIFIED).

Figure 13: ACTOMYOSIN ATPASE CYCLE AS PART OF THE CROSS-BRIDGE CYCLE (BY FITTS 2008, MODIFIED). DIFFERENT CONFIGURATIONAL STATES OF MYOSIN HEADS (COLORED IN BLUE) ARE SHOWN. THE LEVER ARM (COLORED IN RED) CHANGES ITS CONFIGURATION IN PRESENCE OF ATP. MYOSIN FILAMENTS OF THE THICK FILAMENT ARE AT THEIR BASIS (COLORED IN BLUE) ATTACHED TO ACTIN FILAMENTS (YELLOW SPHERES) OF THE THIN FILAMENT.

Figure 14: FEED-FORWARD INHIBITION (LEFT) AND FEEDBACK INHIBITION (RIGHT) (BY KANDEL ET AL., 2012, MODIFIED). MUSCLE DEPICTED REDDISH, CELLS YELLOWISH; AXON ACTIVITY IN DARK YELLOW SHOWS HIGH ACTIVITY, LIGHT YELLOW SHOWS LESS ACTIVITY ACHIEVED BY INHIBITION.

FIGURE 15: DEPICTION OF A CONTROL LOOP WITH FEEDBACK INHIBITION SHOWING CORTICAL DRIVE AND CIRCUITRY IN SPINAL CORD

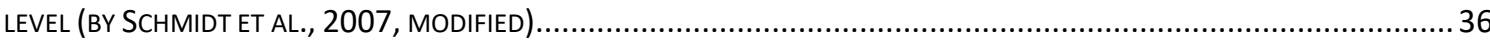

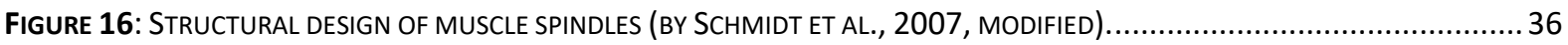


Figures and Tables

FIGURE 17: SCHEMATIC OVERVIEW OF INTER-CONNECTIVITY OF LOWER LIMB AFFERENT AND EFFERENT FIBERS TO ASURE SMOOTH MUSCLE INTERPLAY. RECIPROCAL INHIBITION OF TA BY TS SHOWN EXEMPLARY. AFFERENTS COLORED IN BLUE, EFFERENTS COLORED IN RED.

Figure 18: EXPLANATION OF EXCITATION OF THE MIXED NERVE TN. SHOWN IS THE TIME CURVE OF EMG 100 MS BEFORE AND AFTER THE STIMULATION. UPPER TRACE (RED) DEPICTS DIRECT MOTOR RESPONSE (M-WAVE), LOWER TRACE (GREEN) DEPICTS REFLEX IN THE HOMONYMOUS MUSCLE (H-REFLEX); EXCITATORY STIMULUS APPLIED AT TIME MARK 0 MS.

FIGURE 19: DEPICTION OF RECIPROCAL ACTION IN THE INCREASE IN STIMULATION INTENSITY BETWEEN THE SIZE OF MOTOR RESPONSES OF H-REFLEX (BLACK) AND M-WAVE (GREY) AS MULTIPLES OF MOTOR THRESHOLD, CAUSED BY EXCITATION OF THE PERIPHERAL MIXED NERVE TN (BY KNIKOU, 2008, MODIFIED).

Figure 20: Depiction of THE RELATION BetWEen SIZE OF H-REFLEX AND SIZE OF M-WAVE (BY KNIKOU, 2002, MODIFIED)..........43

Figure 21: Photograph of NINJUTSU GRANDMASTER AND FOUNDER PHD MASAAKI HATSUMI, PERFORMING the A STANCE OF THE SYSTEM BUJINKAN BUDO TAIJUTSU (FROM HTTPS://SHUGYOUDOJO.FILES.WORDPRESS.COM/2011/02/SOKE-KAMAE.JPG

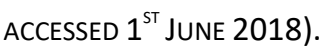

Figure 22: PhOTOGRAPH OF B. K. S. IYENGAR, FOUNDER OF THE STYLE IYENGAR YOGA, PERFORMING A BASIC STRETCH TECHNIQUE (CALLED SUPTA-PĀDĀNGUSHTHĀSANA) ACCESSING ARE MAINLY MUSCLES OF THE POSTERIOR CHAIN (FROM HTTP://WWW.LIVEYOGA.NL/WP-CONTENT/UPLOADS/ 2013/06/SCREEN-SHOT-2013-06-08-AT-8.43.59-AM300X168.PNG ACCESSED $1^{\text {ST }}$ JUNE 2018).

Figure 23: SCHEMATIC OVERVIEW OF THE SUBJECTS' RIGHT LEG WHILE SEATED IN BIODEX SYSTEM 3 PRO ${ }^{\circledR}$ DURING H-REFLEX MEASURE-MENT; EXPLANATION AND ABBREVIATIONS IN FLOATING-TEXT.

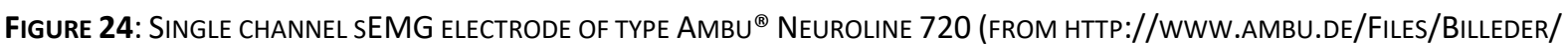

Figure 25: MultichanNel SEMG eleCtrode OF TYPe Bio Elettronica ${ }^{\circledR}$ ELSCH064NM2 .

FIGURE 26: ILLUSTRATION OF HDSEMG RECORDING FROM M. SOLEUS DURING EXPERIMENT; DASHED VERTICAL LINE REPRESENTS END OF RAMP-PART AND BEGINNING OF APPLICATION OF TRIGGER PULSES.

FIGURE 27: EXPLANATION OF DATA ANALYSIS (BY REFERENCE TO HOMONYMOUS EXCITATION) SHOWING MOTOR UNIT DECOMPOSITION IN PERI-STIMULUS DIAGRAMS OF THREE DIFFERENT TYPES: PSTH, PSF AND PSF-CUSUM; STANDARD DEVIATION SHOWN AS RED LINES IN PSF AND PSF-CUSUM; VERTICAL DOTTED LINE REPRESENTS TRIGGER SIGNAL (O MS) AND ONSET OF EXCITATORY REFLEX (APPROX. 42 MS); HORIZONTAL DOTTED LINE REPRESENTS STRENGTH OF EXCITATORY REFLEX;

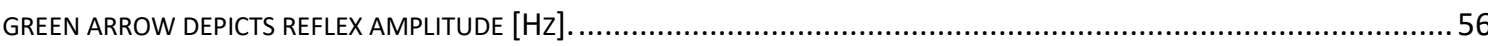

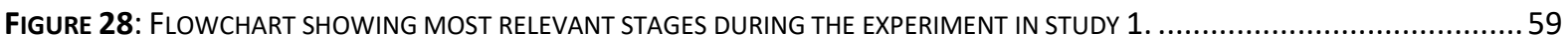

FIGURE 29: EMPIRICAL PROBABILITY DENSITY HISTOGRAM FOR REFLEX AMPLITUDES OF TA, GM AND SOL WITH FITTED GAMMA DISTRIBUTION FOR TA, GM AND SOL AT 10 AND $20 \%$ MVC (BY YAVUZ ET AL., 2018); ABSOLUTE RANGE OF PROBABILITY DENSITY HISTOGRAMS [HZ/STIMULI] WAS APPROXIMATELY FOUR TIMES GREATER FOR TA.

FIGURE 30: A: CUMULATIVE PROBABILITY BETWEEN DISTRIBUTIONS OF REFLEX AMPLITUDES; B: SIGNIFICANCE OF REFLEX AMPLITUDES THROUGH MEDIAN AND RANGE VALUES; MEDIAN REFLEX AMPLITUDES INDICATED BY DASHED VERTICAL LINES (A) AND RED LINES IN BOX PLOTS (B) (BY YAVUZ ET AL., 2018).

Figure 31: CoRRELATION OF RECRUITMENT THRESHOLDS USED TO CALCULATE REFLEX AMPLITUdE (BY YAVUZ ET AL., 2018) .........63

FigURE 32: RECIPROCAL INHIBITORY REFLEX AMPLITUdES [Hz/N.STIM] FOR GM, SOL AND TA, BY YAVUZ ET AL., 2018, MODIFIED; RED LINES INDICATE MEDIAN REFLEX AMPLITUDES, + INDICATES THE AVERAGE REFLEX AMPLITUDES. ................................64

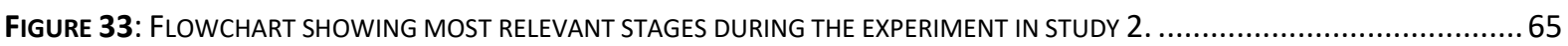

Figure 34: MEDIAN ROOT MEAN SQUARE (RMS) [MV] FROM GM AT 20 \% MVC DURING SUSTAINED CONTRACTION................66

FIGURE 35: MEDIAN ROOT MEAN SQUARE (RMS) [MV] FROM SOL AT $20 \%$ MVC DURING SUSTAINED CONTRACTION ................66

Figure 36: REFLEX AMPLITUdES (PSF-CUSUM PEAK/NUM. OF. STIM.), $10 \%$ MVC FOR GM AND SOL, P < 0.05 FOR ALL

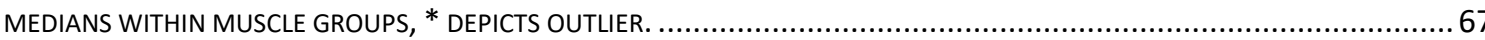

FigURE 37: REFLEX AMPLITUdES (PSF-CUSUM PEAK/NUM. OF. STIM.), 20 \% MVC FOR GM AND SOL, P < 0.05 FOR ALL MEDIANS WITHIN MUSCLE GROUPS, * DEPICTS OUTLIER.

Figure 38:MEAN REFLEX AMPLITUdES (PSF-CUSUM-PEAK/NUM. OF STIM.) OF EARLY AND LATELY RECRUITED MUS AT $10 \%$ MVC FROM SOL 
Figures and Tables

Figure 39: MEAN REFLEX AMPLITUdes (PSF-CUSUM-PEAK/NUM. OF STIM.) OF EARLY AND LATELY RECRUITED MUS AT $20 \%$ MVC FROM SOL

FIGURE 40: SCATTER-PLOT OF REFLEX AMPLITUDES AS A FUNCTION OF RECRUITMENT THRESHOLD OF ALL RECRUITED AND ANALYZED MOTOR UNITS DURING RAMP PART AT $10 \%$ MVC, $\mathrm{N}_{\text {CONTROL }}=69, \mathrm{~N}_{\text {SKILED }}=76$.

FIGURE 41: : SCATTER-PLOT OF REFLEX AMPLITUDES AS A FUNCTION OF RECRUITMENT THRESHOLD OF ALL RECRUITED AND ANALYZED MOTOR UNITS DURING RAMP PART AT $20 \%$ MVC, $\mathrm{N}_{\text {CONTROL }}=98, \mathrm{~N}_{\text {SKILED }}=50$.

FIGURE 42: FLOWCHART SHOWING MOST RELEVANT STAGES DURING THE EXPERIMENT IN STUDY 3.

FIGURE 43 : MEDIAN RELATIVE FORCE OUTPUT COMPARING PRE- AND POST-TEST IN CONTROL AND SKILLED GROUP, PRE-TEST SET AS 1.

FIGURE 44: MEAN MOTOR UNIT DISCHARGE RATE IN RMS [MV] FOR PRE- AND POST-TEST AT 20 \% MVC FOR CONTROL AND SKILLED IN SOL

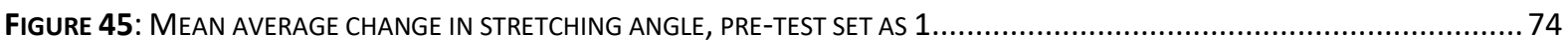

Figure 46: MEDIAN GLOBAL EMG ACTIVITY [MV] FOR PRE- AND POST-TEST AT 50 \% MVC FOR CONTROL AND SKILLED IN SOL. .. 75

FIGURE 47: MEDIAN GLOBAL EMG ACTIVITY [MV] FOR PRE- AND POST-TEST AT 50 \% MVC [MV] FOR CONTROL AND SKILLED IN SOL.

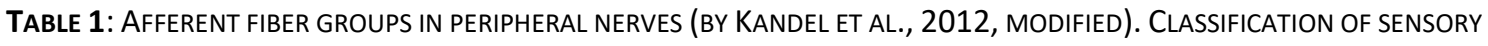
AFFERENTS IN MUSCLE BY THEIR DIAMETER, WHEREAS SENSORY NERVES IN CUTANEOUS NERVES ARE CLASSIFIED ACCORDING THEIR CONDUCTION VELOCITY:

TABLE 2: COMMON CATEGORIZATION OF MOTONEURON TYPES ACCORDING TO CONTRACTION SPEED AND FATIGABILITY (SCHMIDT ET AL., 2007, MODIFIED):

TABLE 3: RECEPTOR TYPES ACTIVE IN SOMATIC SENSATION (BY KANDEL ET AL., 2012, MODIFIED):

TABLE 4: ROOT MEAN SQUARE (RMS) [MV] AND MEDIAN FROM GM AND SOL AT $20 \%$ MVC DURING SUSTAINED CONTRACTION:

TABle 5: VALUES OF BOXPLOT OF REFLEX AMPLITUdeS (PSF-CUSUM PEAK/NUM. OF. STIM.) AT $10 \%$ AND $20 \%$ MVC FOR GM AND SOL:

TABLE 6: MEAN MOTOR UNIT DISCHARGE RATE IN RMS [MV] FOR PRE- AND POST-TEST AT 20 \% MVC FOR CONTROL AND SKILLED IN SOL:

TABLE 7: EXEMPLARY DATA OF ONE SUBJECT SHOWING MEAN AVERAGE CHANGE IN STRETCHING ANGLE:........

TABLE 8: MEAN AND MEDIAN OF ELECTROMECHANICAL DELAY [MS] FOR PRE- AND POST-TEST AT $20 \%$ MVC FOR CONTROL AND SKILLED IN SOL.

TABLE 9: GLOBAL EMG ACTIVITY [MV] FOR PRE- AND POST-TEST AT 50 \% MVC FOR CONTROL AND SKILLED IN SOL: .76 


\section{Abbreviations}

\begin{tabular}{|c|c|}
\hline${ }^{\circ} \mathrm{C}$ & Degree Celsius \\
\hline$\mu \mathrm{m}$ & Micrometer \\
\hline $\mathrm{ACh}$ & Acetylcholine \\
\hline$A D$ & Anno Domini \\
\hline AP & Action potential \\
\hline bit & Bit \\
\hline bps & Bits per second \\
\hline $\mathrm{Ca}^{2+}$ & Calcium ion \\
\hline CNS & Central nervous system \\
\hline CoG & Center of gravity \\
\hline CoM & Center of mass \\
\hline CPN & Common peroneal nerve \\
\hline CUSUM & Cumulative sum \\
\hline DR & Discharge rate \\
\hline EMD & Electromechanical delay \\
\hline EMG & Electromyography \\
\hline EPP & Endplate potential \\
\hline EPSP & Excitatory postsynaptic potential \\
\hline GM & Musculus gastrocnemius \\
\hline HDsEMG & High density surface electromyography \\
\hline H-reflex & Hoffmann-reflex \\
\hline $\mathrm{Hz}$ & Hertz \\
\hline iEMG & Intramuscular electromyography \\
\hline IPSP & Inhibitory postsynaptic potential \\
\hline IQR & Interquartile range \\
\hline $\mathrm{K}^{+}$ & Potassium ion \\
\hline M. & Musculus \\
\hline$m$. & Musculi \\
\hline $\mathrm{mA}$ & Milliampere \\
\hline MAA & Median absolute deviation \\
\hline MDR & Mean discharge rate \\
\hline $\min$ & Minute \\
\hline MN & Motoneuron \\
\hline $\mathrm{ms}$ & Milliseconds \\
\hline MU & Motor unit \\
\hline MUAP & Motor unit action potential \\
\hline MUST & Motor unit spike train \\
\hline $\mathrm{mV}$ & Millivolt \\
\hline MVC & Maximum voluntary contraction \\
\hline MVC & Maximum voluntary contraction \\
\hline M-wave & Direct motor response \\
\hline $\mathrm{Na}^{+}$ & Sodium ion \\
\hline $\mathrm{nm}$ & Nanometer \\
\hline PNS & Peripheral nervous system \\
\hline PSF & Peri-stimulus-frequencygram \\
\hline PSTH & Peri-stimulus-time-histogram \\
\hline & Quartile \\
\hline RMS & Root mean square \\
\hline
\end{tabular}


$S$

sEMG

SMA

SOL

STD

TA

TN

TS

V-wave
Second

Surface electromyography

Supplementary motor area

Musculus soleus

Standard deviation

Musculus tibialis anterior

Tibial nerve

Triceps surae

Voluntary input from higher brain areas 


\section{Literature}

Adkins, D. L.; Boychuk, J.; Remple, M. S.; Kleim, J. a, 2006: Motor training induces experience-specific patterns of plasticity across motor cortex and spinal cord. Journal of applied physiology (Bethesda, Md. : 1985)., 101, 1776-1782.

Almeida-Silveira, M. I.; Pérot, C.; Goubel, F., 1996: Neuromuscular adaptations in rats trained by muscle stretch-shortening. European journal of applied physiology and occupational physiology., 72, 261-266.

Aniss, A. M.; Diener, H. C.; Hore, J.; Burke, D.; Gandevia, S. C., 1990: Reflex activation of muscle spindles in human pretibial muscles during standing. Journal of Neurophysiology., 64, 671-679.

Arus, E., 2013: Biomechanics of human motion : applications in the martial arts. CRC Press.

Augé, W. K.; Morrison, D. S., 2000: Assessment of the infraspinatus spinal stretch reflex in the normal, athletic, and multidirectionally unstable shoulder. The American journal of sports medicine., 28, 206-213.

Avela, J.; Kyröläinen, H.; Komi, P. V, 1999a: Altered reflex sensitivity after repeated and prolonged passive muscle stretching. Journal of applied physiology (Bethesda, Md. : 1985)., 86, 1283-1291.

Avela, J.; Kyröläinen, H.; Komi, P. V; Rama, D., 1999b: Reduced reflex sensitivity persists several days after long-lasting stretch-shortening cycle exercise. Journal of applied physiology (Bethesda, $M d$. : 1985)., 86, 1292-1300.

Awiszus, F.; Feistner, H.; Schäfer, S. S., 1991: On a method to detect long-latency excitations and inhibitions of single hand muscle motoneurons in man. Experimental Brain Research., 86, 440-446.

Balle, S. S.; Magnusson, S. P.; Mchugh, M. P., 2015: Effects of contract-relax vs static stretching on stretch-induced strength loss and length- tension relationship : Stretching ... Effects of contract-relax vs static stretching on stretch-induced strength loss and length - tension relationship.

Behm, D. G.; Blazevich, A. J.; Kay, A. D.; McHugh, M., 2015: Acute effects of muscle stretching on physical performance, range of motion, and injury incidence in healthy active individuals: a systematic review. Applied Physiology, Nutrition, and Metabolism., 11, 1-11.

Bitter, F.; Hillier, S.; Civetta, L., 2011: Change in Dexterity with Sensory Awareness Training: A Randomised Controlled Trial. Perceptual and Motor Skills., 112, 783-798.

Burke, R. E.; Levine, D. N.; Salcman, M.; Tsairis, P., 1974: Motor units in cat soleus muscle: physiological, histochemical and morphological characteristics. The Journal of physiology., 238, 503514.

Capaday, C.; Cody, F. W.; Stein, R. B., 1990: Reciprocal inhibition of soleus motor output in humans during walking and voluntary tonic activity. Journal of neurophysiology., 64, 607-616.

Cappellini, G.; Ivanenko, Y. P.; Poppele, R. E.; Lacquaniti, F., 2006: Motor Patterns in Human Walking and Running. Journal of Neurophysiology., 95, 3426-3437.

Carp, J. S.; Wolpaw, J. R., 1994: Motoneuron plasticity underlying operantly conditioned decrease in primate H-reflex. Journal of neurophysiology., 72, 431-442.

Carp, J. S.; Wolpaw, J. R., 1995: Motoneuron properties after operantly conditioned increase in primate H-reflex. Journal of neurophysiology., 73, 1365-1373. 
Casabona, A.; Polizzi, M. C.; Perciavalle, V., 1990: Differences in H-reflex between athletes trained for explosive contractions and non-trained subjects. European Journal of Applied Physiology and Occupational Physiology., 61, 26-32.

Cavanagh, P. R.; Komi, P. V, 1979: Electromechanical delay in human skeletal muscle under concentric and eccentric contractions. European journal of applied physiology and occupational physiology., 42, 159-163.

Chen, X. Y.; Chen, L.; Wolpaw, J. R., 2001: Time course of H-reflex conditioning in the rat. Neuroscience letters., 302, 85-88.

Chen, X. Y.; Wang, Y.; Chen, Y.; Chen, L.; Wolpaw, J. R., 2016: Ablation of the inferior olive prevents H-reflex down-conditioning in rats. Journal of Neurophysiology., 115, 1630-1636.

Chvatal, S. a; Torres-Oviedo, G.; Safavynia, S. a; Ting, L. H., 2011: Common muscle synergies for control of center of mass and force in nonstepping and stepping postural behaviors. Journal of neurophysiology., 106, 999-1015.

Chvatal, S. a; Ting, L. H., 2013: Common muscle synergies for balance and walking. Frontiers in computational neuroscience., 7, 48.

Cole, K. S.; Curtis, H. J., 1939: ELECTRIC IMPEDANCE OF THE SQUID GIANT AXON DURING ACTIVITY. The Journal of general physiology., 22, 649-670.

Cox, H.; Tilbrook, H.; Aplin, J.; Semlyen, A.; Torgerson, D.; Trewhela, A.; Watt, I., 2010: A randomised controlled trial of yoga for the treatment of chronic low back pain: Results of a pilot study.

Complementary Therapies in Clinical Practice., 16, 187-193.

Cramer, H.; Lauche, R.; Hohmann, C.; Lüdtke, R.; Haller, H.; Michalsen, A.; Langhorst, J.; Dobos, G., 2013: Randomized-controlled Trial Comparing Yoga and Home-based Exercise for Chronic Neck Pain. The Clinical Journal of Pain., 29, 216-223.

Crone, C.; Hultborn, H.; Jespersen, B.; Nielsen, J., 1987: Reciprocal la inhibition between ankle flexors and extensors in man. The Journal of physiology., 389, 163-185.

Crow, E. M.; Jeannot, E.; Trewhela, A., 2015: Effectiveness of lyengar yoga in treating spinal (back and neck) pain: A systematic review. International journal of yoga., 8, 3-14.

D’Agostino, R. B.; Stephens, M. A., 1986: Goodness-of-fit techniques. M. Dekker.

Davey, N. J.; Ellaway, P. H.; Stein, R. B., 1986: Statistical limits for detecting change in the cumulative sum derivative of the peristimulus time histogram. Journal of neuroscience methods., 17, 153-166.

De Carli, F.; Proserpio, P.; Morrone, E.; Sartori, I.; Ferrara, M.; Gibbs, S. A.; De Gennaro, L.; Lo Russo, G.; Nobili, L., 2016: Activation of the motor cortex during phasic rapid eye movement sleep. Annals of neurology., 79, 326-330.

De Luca, C. J.; Adam, A.; Wotiz, R.; Gilmore, L. D.; Nawab, S. H., 2006: Decomposition of surface EMG signals. Journal of neurophysiology., 96, 1646-1657.

Dideriksen, J. L.; Gizzi, L.; Petzke, F.; Falla, D., 2014: Deterministic accessory spinal movement in functional tasks characterizes individuals with low back pain. Clinical Neurophysiology., 125, 16631668.

Duchateau, J.; Baudry, S., 2014: Maximal discharge rate of motor units determines the maximal rate of force development during ballistic contractions in human. Frontiers in human neuroscience., 8, 
234.

Dupeyron, A.; Demattei, C.; Kouyoumdjian, P.; Missenard, O.; Micallef, J. P.; Perrey, S., 2013:

Neuromuscular adaptations after a rehabilitation program in patients with chronic low back pain: case series (uncontrolled longitudinal study). BMC musculoskeletal disorders., 14, 277.

Eccles, J. C.; Fatt, P.; Landgren, S., 1956: Central pathway for direct inhibitory action of impulses in largest afferent nerve fibres to muscle. Journal of neurophysiology., 19, 75-98.

Ehrlenspiel, F.; Wei, K.; Sternad, D., 2010: Open-loop, closed-loop and compensatory control: performance improvement under pressure in a rhythmic task. Experimental Brain Research., 201, 729-741.

Eisenberg, D. M.; Davis, R. B.; Ettner, S. L.; Appel, S.; Wilkey, S.; Van Rompay, M.; Kessler, R. C., 1998: Trends in alternative medicine use in the United States, 1990-1997: results of a follow-up national survey. JAMA., 280, 1569-1575.

Ellaway, P. H., 1978: Cumulative Sum Technique and its application to the analysis of peristimulu time histograms. Electroencephalography and clinical neurophysiology., 45, 302-304.

Enoka, R. M., 2008: Neuromechanics of human movement. Human Kinetics.

Etcheberrigaray, R.; Gibson, G. E.; Alkon, D., 2007: Molecular mechanisms of memory reconsolidation. Nature reviews. Neuroscience., 8, 262-275.

Falco, F. J. E.; Hennessey, W. J.; Goldberg, G.; Braddom, R. L., 1994: H reflex latency in the healthy elderly. Muscle \& Nerve., 17, 161-167.

Farina, D.; Holobar, A.; Merletti, R.; Enoka, R. M., 2010: Decoding the neural drive to muscles from the surface electromyogram. Clinical Neurophysiology., 121, 1616-1623.

Farina, D.; Castronovo, A. M.; Vujaklija, I.; Sturma, A.; Salminger, S.; Hofer, C.; Aszmann, O., 2017: Common Synaptic Input to Motor Neurons and Neural Drive to Targeted Reinnervated Muscles. The Journal of neuroscience : the official journal of the Society for Neuroscience., 37, 11285-11292.

Feldenkrais, M., 1972: Awareness through movement; health exercises for personal growth. Harper \& Row.

Fimland, M. S.; Helgerud, J.; Gruber, M.; Leivseth, G.; Hoff, J., 2009: Functional maximal strength training induces neural transfer to single-joint tasks. European journal of applied physiology., 107, 21-29.

Fitts, R. H., 2007: The cross-bridge cycle and skeletal muscle fatigue. Journal of Applied Physiology., 104, 551-558.

Fogelholm, M.; Malmberg, J.; Suni, J.; Santtila, M.; Kyröläinen, H.; Mäntysaari, M.; Oja, P., 2006: International physical activity questionnaire: Validity against fitness. Medicine and Science in Sports and Exercise., 38, 753-760.

Fukuda, T. Y.; Echeimberg, J. O.; Pompeu, J. E.; Apolinário, A., 2010: Root Mean Square Value of the Electromyographic Signal in the Isometric Torque of the Quadriceps, Hamstrings and Brachial Biceps Muscles in Female, 10, 32-39.

Gatts, S., 2008: Neural mechanisms underlying balance control in Tai Chi. Medicine and sport science., 52, 87-103. 
Goldman, Y. E., 1987: Kinetics of the Actomyosin ATPase in Muscle Fibers. Annual Review of Physiology., 49, 637-654.

Grübel, R.; Erbacher, G.; Larisch, A., 2003: Die Wirksamkeit der Feldenkrais-Methode bei KrebsBetroffenen. Erfahrungsheilkunde., 52, 71-83.

Guissard, N.; Duchateau, J., 2006: Neural Aspects of Muscle Stretching.

Hatsumi, M.; Kuwata, M.; Hirata, M., 2008: Unarmed Fighting Techniques of the Samurai.

Henneman, E.; Somjen, G.; Carpenter, D. O., 1965: Excitability and Inhibitibility of Motoneurons of Different Sizes. Journal of Neurophysiology., 28, 599-620.

Henneman, E., 1977: Functional organization of motoneuron pools: The size principle. In: Asanuma, H. \& V. J. Wilson (eds.), Int. Union Physiol. Sci.,. Igaku-Shoin, Vol. 12p. 50.

Henriksson-Larsén, K. B.; Lexell, J.; Sjöström, M., 1983: Distribution of different fibre types in human skeletal muscles. I. Method for the preparation and analysis of cross-sections of whole tibialis anterior. The Histochemical journal., 15, 167-178.

Hillier, S.; Porter, L.; Jackson, K.; Petkov, J., 2010: The Effects of Feldenkrais Classes on the Health and Function of an Ageing Australian Sample: A Pilot Study !2009-09-14 !2010-03-04 !2010-0413 ! The Open Rehabilitation Journal., 3, 62-66.

Hillier, S.; Worley, A., 2015: The effectiveness of the feldenkrais method: a systematic review of the evidence. Evidence-based complementary and alternative medicine : eCAM., 2015, 752160.

Hirano, M.; Kubota, S.; Tanabe, S.; Koizume, Y.; Funase, K., 2015: Interactions Among Learning Stage, Retention, and Primary Motor Cortex Excitability in Motor Skill Learning. Brain Stimulation., 8, 11951204.

Hodgkin, A. L.; Huxley, A. F., 1952: A quantitative description of membrane current and its application to conduction and excitation in nerve. The Journal of physiology., 117, 500-544.

Hoffmann, P., 1910: Beitrag zur Kenntnis der menschlichen Reflexe mit besonderer Berucksichtigung der elektrischen Erscheinungen. Archiv für Anatomie, Physiologie und Wissenschaftliche Medizin., 1, 223-246.

Holobar, A.; Zazula, D., 2007a: Multichannel blind source separation using convolution Kernel compensation. IEEE Transactions on Signal Processing., 55, 4487-4496.

Holobar, A.; Zazula, D., 2007b: Gradient Convolution Kernel Compensation Applied to Surface Electromyograms. Independent Component Analysis and Signal Separation. Springer Berlin Heidelberg, Berlin, Heidelberg, pp. 617-624.

Holobar, A.; Glaser, V.; Gallego, J. A.; Dideriksen, J. L.; Farina, D., 2012: Non-invasive characterization of motor unit behaviour in pathological tremor. Journal of neural engineering., 9, 056011.

Holobar, A.; Minetto, M. A.; Farina, D., 2014: Accurate identification of motor unit discharge patterns from high-density surface EMG and validation with a novel signal-based performance metric. Journal of neural engineering., 11, 016008.

Hug, F.; Laplaud, D.; Lucia, A.; Grelot, L., 2006: EMG Threshold Determination in Eight Lower Limb Muscles During Cycling Exercise: A Pilot Study. International Journal of Sports Medicine., 27, 456462. 
Hultborn, H.; Pierrot-Deseilligny, E., 1979: Input-output relations in the pathway of recurrent inhibition to motoneurones in the cat. Journal of Physiology., 297, 267-287.

Huxley, A. F.; Niedergerke, R., 1954a: Structural changes in muscle during contraction; interference microscopy of living muscle fibres. Nature., 173, 971-973.

Huxley, H.; Hanson, J., 1954b: Changes in the Cross-Striations of Muscle during Contraction and Stretch and their Structural Interpretation. Nature., 173, 973-976.

Ivanenko, Y. P.; Cappellini, G.; Dominici, N.; Poppele, R. E.; Lacquaniti, F., 2005: Coordination of Locomotion with Voluntary Movements in Humans. Journal of Neuroscience., 25, 7238-7253.

lyengar, B. K. S.; Mangoldt, U. von, 2013: Licht auf Yoga Yoga-Dīpikā ; das grundlegende Lehrbuch des Hatha-Yoga ; mit zahlreichen Übungsprogrammen. Nikol.

Johnson, S. K.; Frederick, J.; Kaufman, M.; Mountjoy, B., 1999: A Controlled Investigation of Bodywork in Multiple Sclerosis. The Journal of Alternative and Complementary Medicine., 5, 237243.

Kandel, E. R.; Schwartz, J. H.; Jessell, T. M.; Siegelbaum, S. A.; Hudspeth, A. J., 2012: Principles of Neural Science Principles of Neural Science Kandel.

Katz, R.; Meunier, S.; Pierrot-Deseilligny, E., 1988: Changes in presynaptic inhibition of la fibres in man while standing. Brain : a journal of neurology., 111, 417-437.

Katz, R.; Penicaud, A.; Rossi, A., 1991: Reciprocal la inhibition between elbow flexors and extensors in the human. The Journal of physiology., 437, 269-286.

Kleim, J. A.; Cooper, N. R.; VandenBerg, P. M., 2002: Exercise induces angiogenesis but does not alter movement representations within rat motor cortex. Brain research., 934, 1-6.

Klintsova, A. Y.; Dickson, E.; Yoshida, R.; Greenough, W. T., 2004: Altered expression of BDNF and its high-affinity receptor TrkB in response to complex motor learning and moderate exercise. Brain Research., 1028, 92-104.

Knikou, M., 2008: The H-reflex as a probe: Pathways and pitfalls. Journal of Neuroscience Methods., $171,1-12$.

Koceja, D. M.; Davison, E.; Robertson, C. T., 2004: Neuromuscular characteristics of endurance- and power-trained athletes. Research quarterly for exercise and sport., 75, 23-30.

Kolt, G. S.; McConville, J. C., 2000: The effects of a Feldenkrais ${ }^{\circledR}$ Awareness Through Movement program on state anxiety. Journal of Bodywork and Movement Therapies., 4, 216-220.

Lacquaniti, F.; Ivanenko, Y. P.; Zago, M., 2012: Patterned control of human locomotion. The Journal of Physiology., 590, 2189-2199.

Laporte, Y.; Lloyd, D. P. C., 1952: Nature and Significance of the Reflex Connections Established by Large Afferent Fibers of Muscular Origin. American Journal of Physiology-Legacy Content., 169, 609621.

Laumer, U.; Bauer, M.; Fichter, M.; Milz, H., 1997: Therapeutic effects of the Feldenkrais method \&quot;awareness through movement\&quot; in patients with eating disorders. Psychotherapie, Psychosomatik, medizinische Psychologie., 47, 170-180.

Lavoie, B. A.; Devanne, H.; Capaday, C., 1997: Differential control of reciprocal inhibition during 
walking versus postural and voluntary motor tasks in humans. Journal of neurophysiology., 78, 429438.

Lexell, J.; Jarvis, J. C.; Currie, J.; Downham, D. Y.; Salmons, S., 1994: Fibre type composition of rabbit tibialis anterior and extensor digitorum longus muscles. Journal of anatomy., 185 ( Pt 1), 95-101.

Liddell, E. G. T.; Sherrington, C., 1924: Reflexes in Response to Stretch (Myotatic Reflexes). Proceedings of the Royal Society of London. Series B, Containing Papers of a Biological Character.

Lohmann, C.; Kessels, H. W., 2014: The developmental stages of synaptic plasticity. The Journal of physiology., 592, 13-31.

Loram, I. D.; Gollee, H.; Lakie, M.; Gawthrop, P. J., 2011: Human control of an inverted pendulum: is continuous control necessary? Is intermittent control effective? Is intermittent control physiological? The Journal of physiology., 589, 307-324.

Maffiuletti, N. A.; Martin, A.; Babault, N.; Pensini, M.; Lucas, B.; Schieppati, M., 2001: Electrical and mechanical Hmax-to-Mmaxratio in power- and endurance-trained athletes. Journal of Applied Physiology., 90, 3-9.

Magnuson, P. B., 1944: Differential Diagnosis of Causes of Pain in the Lower Back Accompanied by Sciatic Pain. Annals of surgery., 119, 878-891.

Matsuo, S.; Suzuki, S.; Iwata, M.; Banno, Y.; Asai, Y.; Tsuchida, W.; Inoue, T., 2013: Acute Effects of Different Stretching Durations on Passive Torque, Mobility, and Isometric Muscle Force. Journal of Strength and Conditioning Research., 27, 3367-3376.

Mazzocchio, R.; Kitago, T.; Liuzzi, G.; Wolpaw, J. R.; Cohen, L. G., 2006: Plastic changes in the human H-reflex pathway at rest following skillful cycling training. Clinical Neurophysiology., 117, 1682-1691.

Michalsen, A.; Traitteur, H.; Lüdtke, R.; Brunnhuber, S.; Meier, L.; Jeitler, M.; Büssing, A.; Kessler, C., 2012: Yoga for Chronic Neck Pain: A Pilot Randomized Controlled Clinical Trial. The Journal of Pain., 13, 1122-1130.

Miles, T. S.; Türker, K. S., 1986: Does reflex inhibition of motor units follow the 'size principle'? Experimental brain research., 62, 443-445.

Milnik, V., 2012: Elektrophysiologie in der Praxis Neurographie, evozierte Potenziale und EEG. Elsevier, Urban \& Fischer.

Misiaszek, J. E., 2003: The H-reflex as a tool in neurophysiology: Its limitations and uses in understanding nervous system function. Muscle and Nerve., 28, 144-160.

Murray, J. M., 1939: Lower Back Pain. Canadian Medical Association journal., 41, 427-434.

Myers, T. W., 2013: Anatomy trains : myofascial meridians for manual and movement therapists.

Negro, F.; Muceli, S.; Castronovo, A. M.; Holobar, A.; Farina, D., 2016: Multi-channel intramuscular and surface EMG decomposition by convolutive blind source separation. Journal of Neural Engineering., 13, 026027.

Ni, M.; Mooney, K.; Balachandran, A.; Richards, L.; Harriell, K.; Signorile, J. F., 2014: Muscle utilization patterns vary by skill levels of the practitioners across specific yoga poses (asanas). Complementary Therapies in Medicine., 22, 662-669.

Nielsen, J.; Kagamihara, Y., 1992: The regulation of disynaptic reciprocal la inhibition during co- 
contraction of antagonistic muscles in man. The Journal of physiology., 456, 373-391.

Nielsen, J.; Kagamihara, Y., 1993a: The regulation of presynaptic inhibition during co-contraction of antagonistic muscles in man. The Journal of physiology., 464, 575-593.

Nielsen, J.; Nagaoka, M.; Kagamihara, Y.; Kakuda, N.; Tanaka, R., 1994: Discharge of muscle afferents during voluntary co-contraction of antagonistic ankle muscles in man. Neuroscience letters., 170, 277-280.

Nielsen, J. B.; Crone, C.; Hultborn, H., 1993b: H-reflexes are smaller in dancers from The Royal Danish Ballet than in well-trained athletes. European Journal of Applied Physiology and Occupational Physiology., 66, 116-121.

Nielsen, J. B., 2016: Human Spinal Motor Control. Annual Review of Neuroscience., 39, 81-101.

Ogawa, T.; Kim, G. H.; Sekiguchi, H.; Akai, M.; Suzuki, S.; Nakazawa, K., 2009: Enhanced stretch reflex excitability of the soleus muscle in experienced swimmers. European Journal of Applied Physiology., 105, 199-205.

Oliveira, A. S.; Silva, P. B.; Lund, M. E.; Gizzi, L.; Farina, D.; Kersting, U. G., 2013: Effects of Perturbations to Balance on Neuromechanics of Fast Changes in Direction during Locomotion. PloS one., 8, e59029.

Oliveira, A. S. C.; Gizzi, L.; Kersting, U. G.; Farina, D., 2012: Modular organization of balance control following perturbations during walking. Journal of neurophysiology., 108, 1895-1906.

Palmieri, R. M.; Ingersoll, C. D.; Hoffman, M. A., 2004: The hoffmann reflex: methodologic considerations and applications for use in sports medicine and athletic training research. Journal of athletic training., 39, 268-277.

Pape, H. C.; Kurtz, A.; Silbernagl, S., 2014: Physiologie. Thieme.

Pascual-Leone, A.; Cammarota, A.; Wassermann, E. M.; Brasil-Neto, J. P.; Cohen, L. G.; Hallett, M., 1993: Modulation of motor cortical outputs to the reading hand of braille readers. Annals of Neurology., 34, 33-37.

Perez, M. A.; Lundbye-Jensen, J.; Nielsen, J. B., 2007: Task-specific depression of the soleus H-reflex after cocontraction training of antagonistic ankle muscles. Journal of neurophysiology., 98, 36773687.

Perl, E. R., 1962: A comparison of monosynaptic and polysynaptic reflex responses from individual flexor motoneurones. The Journal of Physiology., 164, 430-449.

Petersen, N.; Morita, H.; Nielsen, J., 1999: Modulation of reciprocal inhibition between ankle extensors and flexors during walking in man. The Journal of physiology., 520 Pt 2, 605-619.

Pierrot-Deseilligny, E.; Mazevet, D., 2000: The monosynaptic reflex: A tool to investigate motor control in humans. Interest and limits. Neurophysiologie Clinique., 30, 67-80.

Pierrot-Deseilligny, E.; Burke, D., 2012: The Circuitry of the Human Spinal Cord. Cambridge University Press; 1st edition. Yale Journal of Biology and Medicine, Vol. 88.

Pratt, C. A.; Jordan, L. M., 1987: la inhibitory interneurons and Renshaw cells as contributors to the spinal mechanisms of fictive locomotion. Journal of Neurophysiology., 57, 56-71.

Prochazka, A.; Ellaway, P., 2012: Sensory systems in the control of movement. Comprehensive 
Physiology., 2, 2615-2627.

Pröttel, B., 2017: Der Effekt von langfristigen Dehnübungen im Yoga auf die spinale Plastizität und die Erregbarkeit von motorischen Einheiten und die daraus resultierenden neuronalen und funktionellen Vorteile - eine Untersuchung der unteren Extremität. Georg-August-Universität Göttingen.

Purves, D.; Augustine, G. J.; Fitzpatrick, D.; Katz, L. C.; LaMantia, A. S.; McNamara, J. O.; Williams, S. M., 2001: The Motor Unit.

Pyndt, H. S.; Nielsen, J. B., 2003: Modulation of Transmission in the Corticospinal and Group la Afferent Pathways to Soleus Motoneurons During Bicycling. Journal of Neurophysiology., 89, 304314.

Quintero, Y.; Restrepo, C. C.; Tamayo, V.; Tamayo, M.; Vélez, A. L.; Gallego, G.; PelÁez-Vargas, A., 2009: Effect of awareness through movement on the head posture of bruxist children. Journal of Oral Rehabilitation., 36, 18-25.

Remple, M. S.; Bruneau, R. M.; VandenBerg, P. M.; Goertzen, C.; Kleim, J. a., 2001: Sensitivity of cortical movement representations to motor experience: evidence that skill learning but not strength training induces cortical reorganization. Behavioural Brain Research., 123, 133-141.

Robertson, C. T.; Kitano, K.; Koceja, D. M.; Riley, Z. A., 2012: Temporal depression of the soleus Hreflex during passive stretch. Experimental brain research., 219, 217-225.

Rochcongar, P.; Dassonville, J.; Le Bars, R., 1979: Modification of the Hoffmann reflex in function of athletic training (author's transl). European journal of applied physiology and occupational physiology., 40, 165-170.

Rogasch, N. C.; Burne, J. A.; Turker, K. S.; Türker, K. S., 2012: Comparison of the inhibitory response to tendon and cutaneous afferent stimulation in the human lower limb. Journal of Neurophysiology., $107,564-572$.

Ruth, S.; Kegerreis, S., 1992: Facilitating Cervical Flexion Using a Feldenkrais Method: Awareness through Movement. Journal of Orthopaedic \& Sports Physical Therapy., 16, 25-29.

Safavynia, S. A.; Torres-Oviedo, G.; Ting, L. H., 2011: Muscle Synergies: Implications for Clinical Evaluation and Rehabilitation of Movement. Topics in spinal cord injury rehabilitation., 17, 16-24.

Sale, D. G.; MacDougall, J. D.; Upton, A. R.; McComas, A. J., 1983: Effect of strength training upon motoneuron excitability in man. Medicine and science in sports and exercise., 15, 57-62.

Sawers, A.; Allen, J. L.; Ting, L. H., 2015: Long-term training modifies the modular structure and organization of walking balance control. Journal of Neurophysiology., jn.00758.2015.

Schiebler, T. H.; Korf, H. W., 2007: Anatomie : Histologie, Entwicklungsgeschichte, makroskopische und mikroskopische Anatomie, Topographie ; unter Berücksichtigung des Gegenstandskatalogs ; mit 111 Tabellen. Steinkopff.

Schiller, D.; Phelps, E. a, 2011: Does reconsolidation occur in humans? Frontiers in behavioral neuroscience., 5, 24.

Schmidt, R. F.; Lang, F.; Thews, G., 2007: Physiologie des Menschen mit Pathophysiologie. Book., Vol. 29.

Schneider, C.; Capaday, C., 2002: Progressive Adaptation of the Soleus H-Reflex With Daily Training 
at Walking Backward. Journal of Neurophysiology., 648-656.

Schubert, M.; Beck, S.; Taube, W.; Amtage, F.; Faist, M.; Gruber, M., 2008: Balance training and ballistic strength training are associated with task-specific corticospinal adaptations. European Journal of Neuroscience., 27, 2007-2018.

Schwabe, L.; Nader, K.; Pruessner, J. C., 2014: Reconsolidation of human memory: Brain mechanisms and clinical relevance. Biological Psychiatry., 76, 274-280.

Segal, R. L.; Wolf, S. L.; Catlin, P. A.; Gilliand, R. L.; Taffs, J. K.; Bass, H. C.; Vickers, E. F., 2000: Uncoupling of human short and long latency stretch reflex responses with operant conditioning. Restorative neurology and neuroscience., 17, 17-22.

Sforza, C.; Turci, M.; Grassi, G. P.; Shirai, Y. F.; Pizzini, G.; Ferrario, V. F., 2002: Repeatability of maegeri-keage in traditional karate: a three-dimensional analysis with black-belt karateka. Perceptual and motor skills., 95, 433-444.

Sørensen, H.; Zacho, M.; Simonsen, E. B.; Dyhre-Poulsen, P.; Klausen, K., 1996: Dynamics of the martial arts high front kick. Journal of sports sciences., 14, 483-495.

Stephens, J.; Davidson, J.; DeRosa, J.; Kriz, M.; Saltzman, N., 2006: Lengthening the Hamstring Muscles Without Stretching Using \&quot;Awareness Through Movement\&quot; Physical Therapy., 86, 1641-1650.

Swain, R. A.; Harris, A. B.; Wiener, E. C.; Dutka, M. V; Morris, H. D.; Theien, B. E.; Konda, S.; Engberg, K.; Lauterbur, P. C.; Greenough, W. T., 2003: Prolonged exercise induces angiogenesis and increases cerebral blood volume in primary motor cortex of the rat. Neuroscience., 117, 1037-1046.

Thompson, A. K.; Chen, X. Y.; Wolpaw, J. R., 2009: Acquisition of a simple motor skill: task-dependent adaptation plus long-term change in the human soleus $\mathrm{H}$-reflex. The Journal of neuroscience., 29, 5784-5792.

Thompson, A. K.; Wolpaw, J. R., 2014: The simplest motor skill: mechanisms and applications of reflex operant conditioning. Exercise and sport sciences reviews., 42, 82-90.

Tilbrook, H. E.; Cox, H.; Hewitt, C. E.; Kang'ombe, A. R.; Chuang, L. H.; Jayakody, S.; Aplin, J. D.; Semlyen, A.; Trewhela, A.; Watt, I.; Torgerson, D. J., 2011: Yoga for Chronic Low Back Pain. Annals of Internal Medicine., 155, 569.

Ting, L. H.; McKay, J. L., 2007: Neuromechanics of muscle synergies for posture and movement. Current Opinion in Neurobiology., 17, 622-628.

Türker, K. S.; Cheng, H. B., 1994: Motor-unit firing frequency can be used for the estimation of synaptic potentials in human motoneurones. Journal of Neuroscience Methods., 53, 225-234.

Türker, K. S.; Schmied, A.; Cheng, H. B., 1996: Correlated changes in the firing rate of human motor units during voluntary contraction. Experimental Brain Research., 111, 455-464.

Tyč, F.; Boyadjian, A.; Devanne, H., 2005: Motor cortex plasticity induced by extensive training revealed by transcranial magnetic stimulation in human. European Journal of Neuroscience., 21, 259-266.

Ung, R. V.; Imbeault, M. A.; Ethier, C.; Brizzi, L.; Capaday, C., 2005: On the Potential Role of the Corticospinal Tract in the Control and Progressive Adaptation of the Soleus H-Reflex During Backward Walking. Journal of Neurophysiology., 94, 1133-1142. 
Vaisy, M.; Gizzi, L.; Petzke, F.; Consmüller, T.; Pfingsten, M.; Falla, D., 2015: Measurement of Lumbar Spine Functional Movement in Low Back Pain. The Clinical Journal of Pain., 31, 876-885.

Vila-Cha, C.; Falla, D.; Correia, M. V.; Farina, D., 2012: Changes in H reflex and V wave following short-term endurance and strength training. Journal of Applied Physiology., 112, 54-63.

Vrantsidis, F.; Hill, K. D.; Moore, K.; Webb, R.; Hunt, S.; Dowson, L., 2009: Getting Grounded Gracefully: effectiveness and acceptability of Feldenkrais in improving balance. Journal of aging and physical activity., 17, 57-76.

Walker, M. P.; Brakefield, T.; Hobson, J. A., 2003: Dissociable stages of human memory consolidation and reconsolidation. Nature., 425, 616-620.

Walton, D. M.; Kuchinad, R. a; Ivanova, T. D.; Garland, S. J.; Garland, J., 2002: Reflex inhibition during muscle fatigue in endurance-trained and sedentary individuals. European journal of applied physiology., 87, 462-468.

Webb, M. R.; Trentham, D. R.; Webb, M. R.; Trentham, D. R., 2011: Chemical Mechanism of MyosinCatalyzed ATP Hydrolysis. Comprehensive Physiology. John Wiley \& Sons, Inc., Hoboken, NJ, USA.

Widmaier, E. P.; Raff, H.; Strang, K. T.; Vander, A. J., 2013: Vander's human physiology : the mechanisms of body function.

Williams, K.; Abildso, C.; Steinberg, L.; Doyle, E.; Epstein, B.; Smith, D.; Hobbs, G.; Gross, R.; Kelley, G.; Cooper, L., 2009: Evaluation of the Effectiveness and Efficacy of Iyengar Yoga Therapy on Chronic Low Back Pain. Spine., 34, 2066-2076.

Williams, K. A.; Petronis, J.; Smith, D.; Goodrich, D.; Wu, J.; Ravi, N.; Doyle, E. J.; Juckett, G. R.; Kolar, M. M.; Gross, R.; Steinberg, L., 2005: Effect of lyengar yoga therapy for chronic low back pain. Pain., 115, 107-117.

Williams, K. A., 2009: Evaluation of the effectiveness and efficacy of lyengar Yoga therapy on chronic low back pain: Kommentar. Journal fur Mineralstoffwechsel., 16, 197.

Wolpaw, J. R.; Lee, C. L., 1989: Memory traces in primate spinal cord produced by operant conditioning of H-reflex. Journal of neurophysiology., 61, 563-572.

Wolpaw, J. R., 2006: The education and re-education of the spinal cord. Progress in brain research., 157, 261-280.

Wolpaw, J. R., 2007: Spinal cord plasticity in acquisition and maintenance of motor skills. Acta physiologica., 189, 155-169.

Wymbs, N. F.; Bastian, A. J.; Celnik, P. A.; Wymbs, N. F.; Bastian, A. J.; Celnik, P. A., 2016: Motor Skills Are Strengthened through Motor Skills Are Strengthened through Reconsolidation. Current Biology., 26, 1-6.

Yavuz, U. S.; Sendemir-Urkmez, A.; Türker, K. S., 2010: Effect of gender, age, fatigue and contraction level on electromechanical delay. Clinical Neurophysiology., 121, 1700-1706.

Yavuz, U. S.; Mrachacz-Kersting, N.; Sebik, O.; Berna Unver, M.; Farina, D.; Turker, K. S., 2014: Human stretch reflex pathways reexamined. Journal of Neurophysiology., 111, 602-612.

Yavuz, U. S.; Negro, F.; Sebik, O.; Holobar, A.; Frömmel, C.; Türker, K. S.; Farina, D., 2015: Estimating reflex responses in large populations of motor units by decomposition of the high-density surface electromyogram. The Journal of Physiology., 593, 4305-4318. 
Yavuz, U. S.; Negro, F.; Diedrichs, R.; Türker, K. S.; Farina, D., 2017: Reflex Circuitry Originating from the Muscle Spindles to the Tibialis Anterior Muscle. Converging Clinical and Engineering Research on Neurorehabilitation II. Springer, pp. 177-181.

Yavuz, U. S. Ş.; Negro, F. F.; Diedrichs, R.; Farina, D., 2018: Reciprocal inhibition between motor neurons of the tibialis anterior and triceps surae in humans. Journal of neurophysiology., 119, 16991706.

Zaggelidis, G.; Lazaridis, S., 2013: Muscle Activation Profiles of Lower Extremities in Different Throwing Techniques and in Jumping Performance in Elite and Novice Greek Judo Athletes. Journal of human kinetics., 37, 63-70.

Zehr, E. P.; Sale, D. G.; Dowling, J. J., 1997: Ballistic movement performance in karate athletes. Medicine and science in sports and exercise., 29, 1366-1373.

Zotz, T. G.; Capriglione, L. G. A.; Zotz, R.; Noronha, L.; Viola De Azevedo, M. L.; Fiuza Martins, H. R.; Silveira Gomes, A. R., 2016: Acute effects of stretching exercise on the soleus muscle of female aged rats. Acta histochemica., 118, 1-9. 
Curriculum Vitae

\section{Curriculum Vitae}

\section{Personal Data}

Name Robin Diedrichs

Date of birth March $27^{\text {th }} 1985$

Place of birth Salzgitter-Bad

Address Leinestraße 12, 37073 Göttingen

E-mail r.diedrichs@gmx.net 
Curriculum Vitae 
Curriculum Vitae 


\section{Publications related to this thesis}

Yavuz, U. S. Ş.; Negro, F. F.; Diedrichs, R.; Farina, D., 2018: Reciprocal inhibition between motor neurons of the tibialis anterior and triceps surae in humans. Journal of neurophysiology., 119, 16991706. 


\section{Acknowledgements}

Als erstes möchte ich meinem Betreuer Herrn Prof. Dr. Heinrich danken, mit dessen kontinuierlicher Unterstützung ich die Doktorarbeit erst planen, durchführen und abschließen konnte.

Herrn Prof. Dr. Farina danke ich für die wissenschaftliche Unterstützung meiner Promotion und die Aufnahme in die Abteilung des Instituts für Neurorehabilitationssysteme.

Dem Institut für Neurorehabilitationssysteme danke ich für die Unterstützung meines Forschungsvorhabens. Im Speziellen gilt mein Dank Herrn Dr. Utku Yavuz für die wissenschaftliche und praktische Unterstützung und Herrn Dr. Rok Istenic für die wissenschaftliche und zwischenmenschliche Unterstützung während meiner Promotion.

Herrn Prof. Dr. Schilling und der Universitätsmedizin Göttingen danke ich für die wissenschaftliche Weiterführung meiner Promotion nach dem Weggang von Herrn Prof. Dr. Farina.

Ein ganz besonderer Dank gilt Frau Prof. Dr. Kersten für die starke Unterstützung im Verlauf der Promotion und für die konstruktiven und aufbauenden Worte.

Bei Herrn Prof. Dr. Frömmel möchte ich mich für die Unterstützung in der konkreten Startphase meiner Promotion und für die angenehmen Gespräche und das Interesse an meiner Arbeit bedanken.

In dem Vorbereitungs- und Ideenfindungsprozess meiner Arbeit haben mich Herr Prof. Dr. Thienes und Herr Prof. Dr. Nitsche wesentlich unterstützt und mir mir Möglichkeiten und Ansprechpartner aufgezeigt, wofür ich mich herzlich bedanken möchte.

Ohne das Bujinkan Arashi Dojo Göttingen hätte sich vermutlich die Idee zur Grundlage dieser Promotion nicht gebildet. Durch die Möglichkeit das Verhalten der Teilnehmer des Dojos in Experimenten zu messen, konnte ich wesentliche Teile meiner Doktorarbeit überhaupt erst bearbeiten und es gibt noch viele offene und weiterführende Fragen, die wissenschaftlich beantwortet werden müssten.

Kai, Joachim und Hajo danke ich für die langjährige aufrichtige Unterstützung, egal in welchen Dingen wir diese und uns gegenseitig benötigen.

Lennart danke ich für die vielen aufbauenden und konstruktiven Worte und Gespräche und den Glauben an und in mich, während der gesamten Promotion und in der Zeit der Umstrukturierung des Institutes.

Meiner Familie, als meinen Eltern Anna-Katharina und Dr. Ulrich Diedrichs, und meinem Bruder Falko Diedrichs danke ich für die langjährige Unterstützung meines beruflichen Werdegangs und dafür, dass sie immer für mich da waren und sind. 